\title{
Determining the Impact of Ligand and Alkene Substituents on Bonding in Gold(I)-Alkene Complexes Supported by N- Heterocyclic Carbenes: A Computational Study
}

\author{
John T. York* \\ Department of Chemistry, Stetson University, \\ 421. N. Woodland Blvd. \\ DeLand, FL 32723 \\ *Corresponding Author: jyork@ stetson.edu \\ Phone: 386-822-8181
}

Fax: 386-740-3601 
(a)

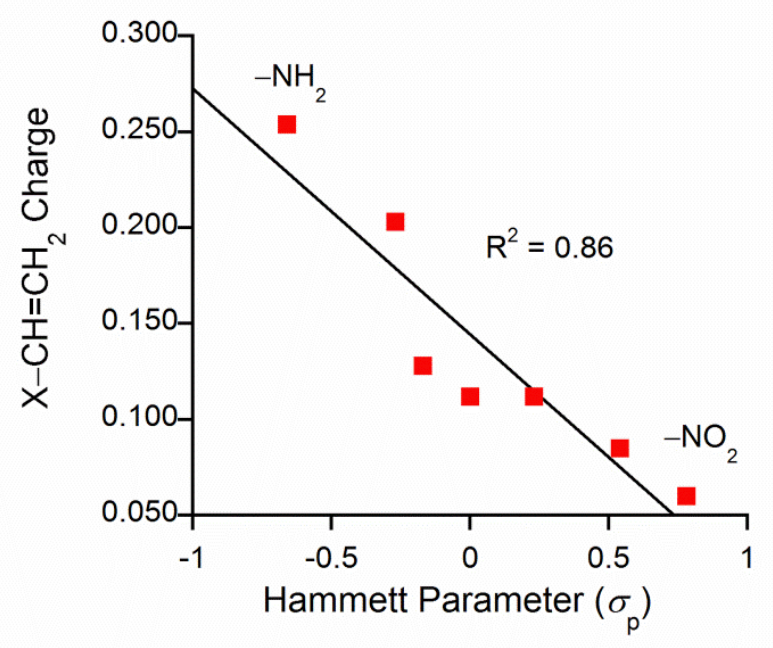

(b)

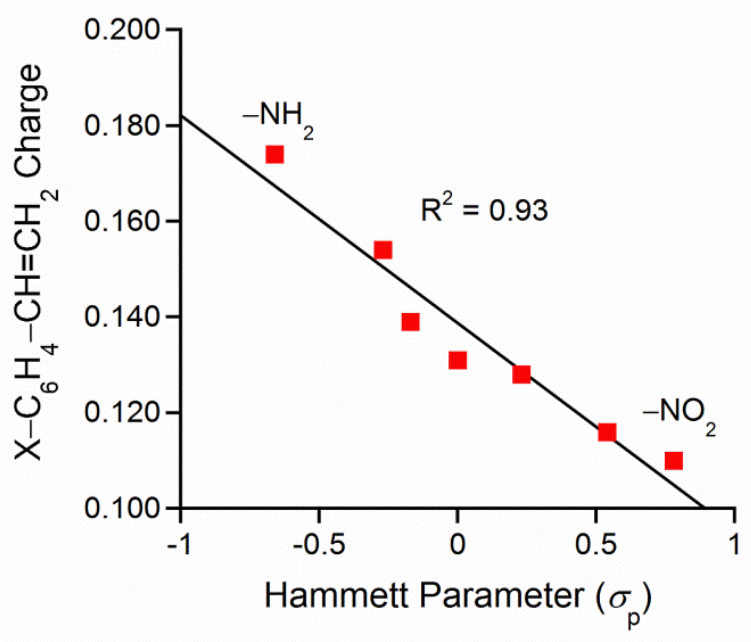

Figures S1a and S1b. Alkene charge versus Hammett parameter $\left(\sigma_{\mathrm{p}}\right)$ for (a) $[(\mathrm{NHC}) \mathrm{Au}(\mathrm{X}-$ $\left.\left.\mathrm{CH}=\mathrm{CH}_{2}\right)\right]^{+}$complexes $\mathbf{2 a}-\mathbf{g}$ and (b) $\left[(\mathrm{NHC}) \mathrm{Au}\left(\mathrm{X}-\mathrm{C}_{6} \mathrm{H}_{4}-\mathrm{CH}=\mathrm{CH}_{2}\right)\right]^{+}$complexes $\mathbf{3 a}-\mathbf{g}$.

(a)

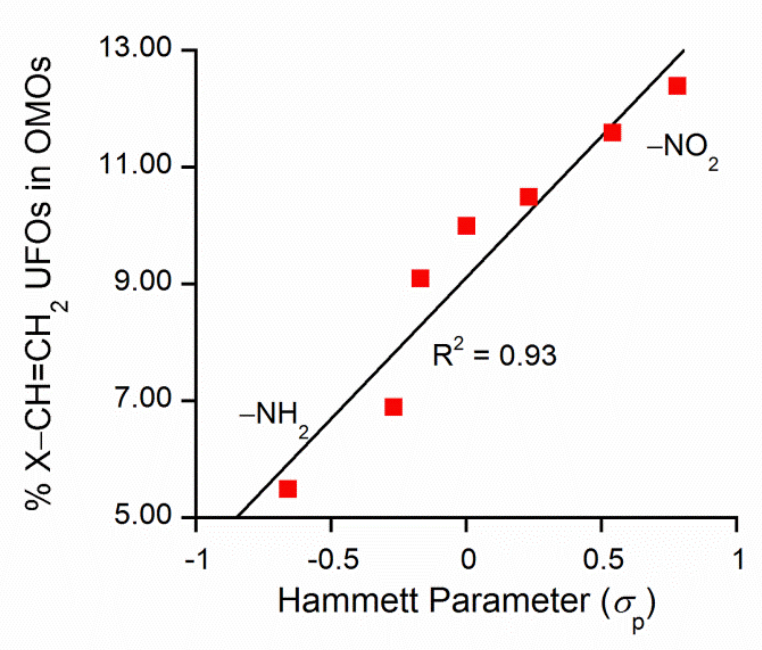

(b)

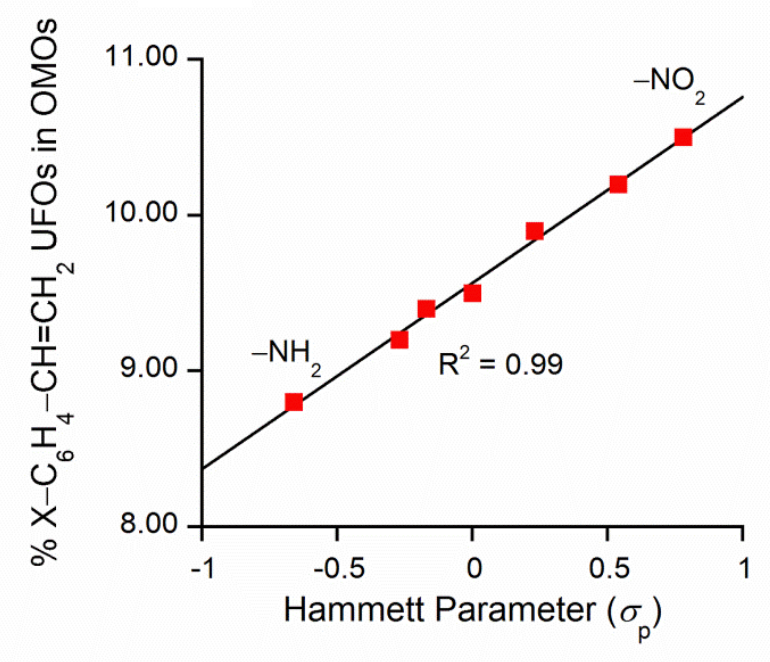

Figures S2a and S2b. Percent (\%) alkene unoccupied fragment orbitals (UFOs) in occupied molecular orbitals (OMOs) versus Hammett parameter $\left(\sigma_{\mathrm{p}}\right)$ for $(\mathrm{a})\left[(\mathrm{NHC}) \mathrm{Au}\left(\mathrm{X}-\mathrm{CH}=\mathrm{CH}_{2}\right)\right]^{+}$ complexes $\mathbf{2 a}-\mathbf{g}$, and (b) $\left[(\mathrm{NHC}) \mathrm{Au}\left(\mathrm{X}-\mathrm{C}_{6} \mathrm{H}_{4}-\mathrm{CH}=\mathrm{CH}_{2}\right)\right]^{+}$complexes $\mathbf{3 a}-\mathbf{g}$. 
(a)

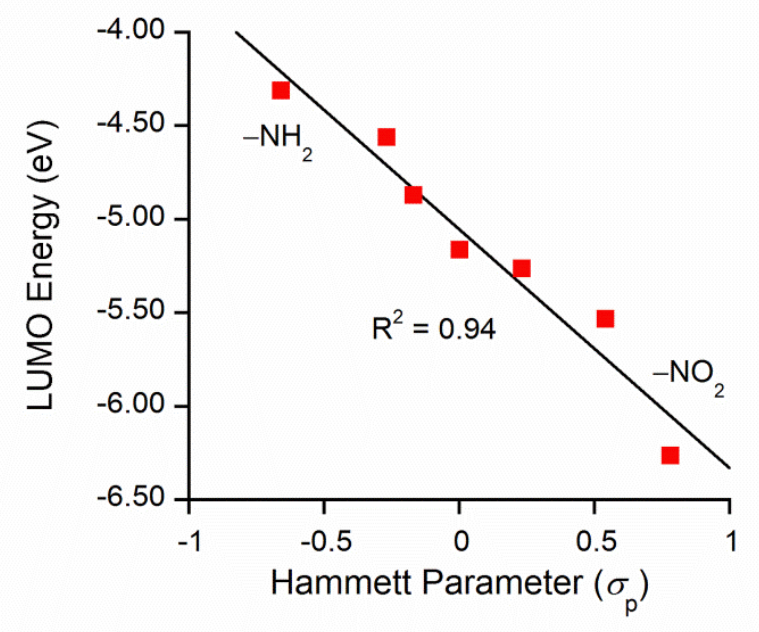

(b)

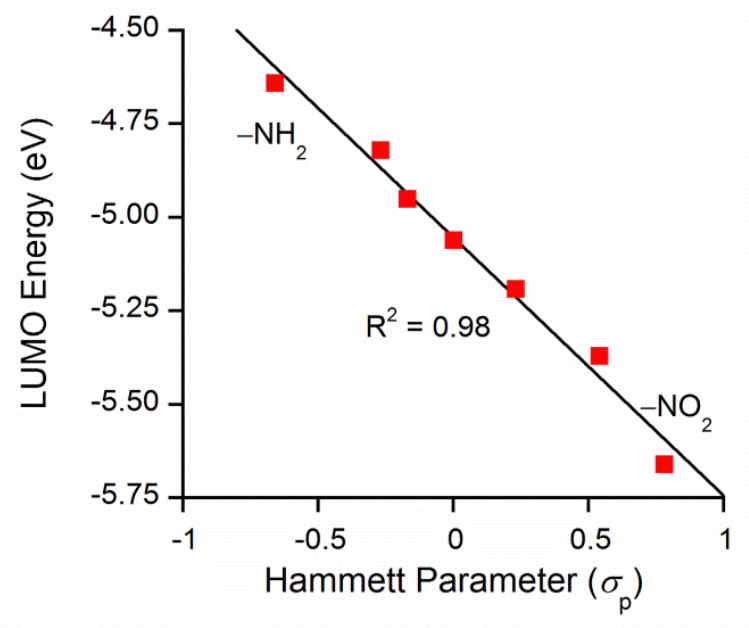

Figures S3a and S3b. Calculated LUMO energy $(\mathrm{eV})$ versus Hammett parameter $\left(\sigma_{\mathrm{p}}\right)$ for $(\mathrm{a})$ $\left[(\mathrm{NHC}) \mathrm{Au}\left(\mathrm{X}-\mathrm{CH}=\mathrm{CH}_{2}\right)\right]^{+}$complexes $\mathbf{2 a}-\mathbf{g}$, and (b) $\left[(\mathrm{NHC}) \mathrm{Au}\left(\mathrm{X}-\mathrm{C}_{6} \mathrm{H}_{4}-\mathrm{CH}=\mathrm{CH}_{2}\right)\right]^{+}$complexes $\mathbf{3 a}-\mathbf{g}$.

(a)

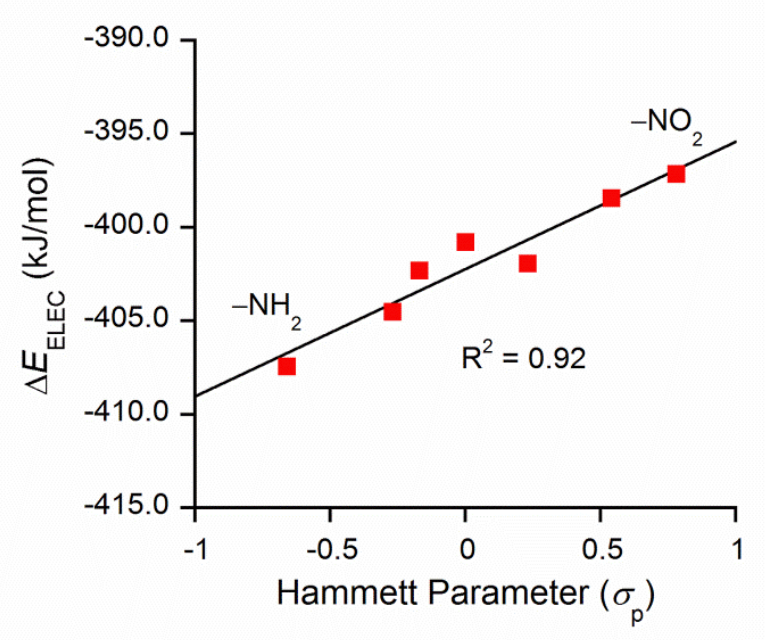

(b)

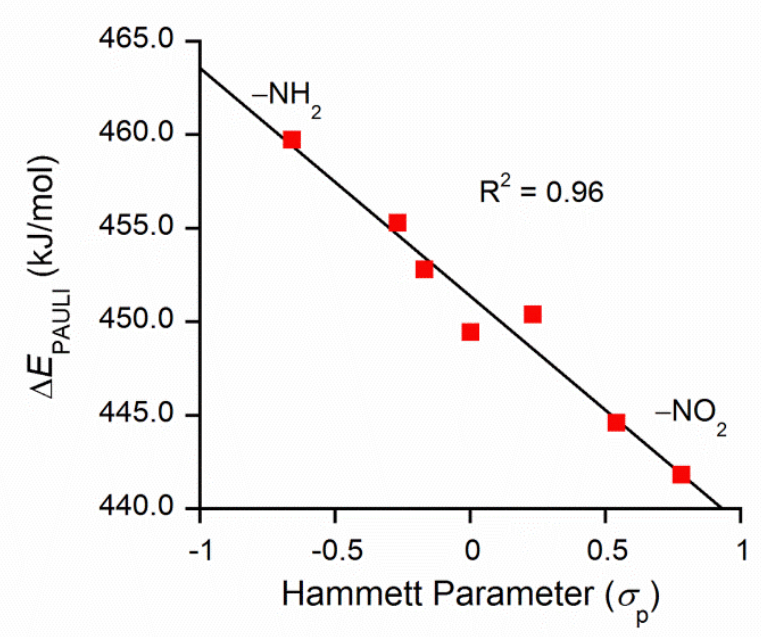

Figures S4a and S4b. EDA-NOCV calculated values for (a) $\Delta E_{\mathrm{ELEC}}$ and (b) $\Delta E_{\mathrm{PAULI}}$ versus Hammett parameter $\left(\sigma_{\mathrm{p}}\right)$ for $\left[(\mathrm{X}-\mathrm{NHC}) \mathrm{Au}\left(\mathrm{C}_{2} \mathrm{H}_{4}\right)\right]^{+}$complexes $\mathbf{1 a}-\mathbf{g}$. 
(a)

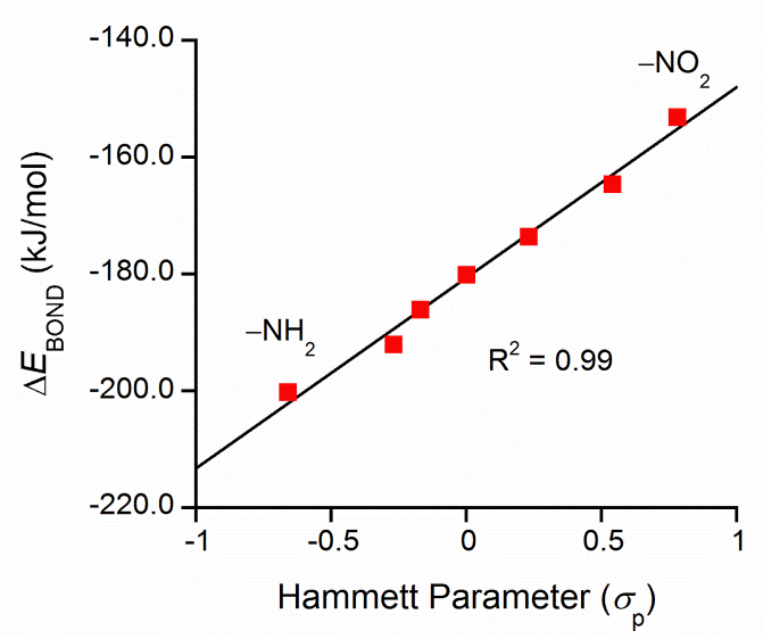

(c)

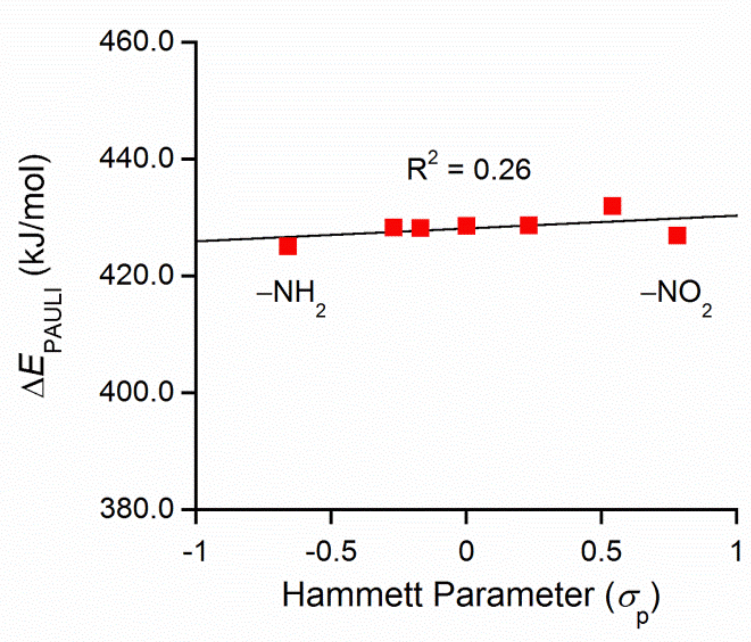

(b)

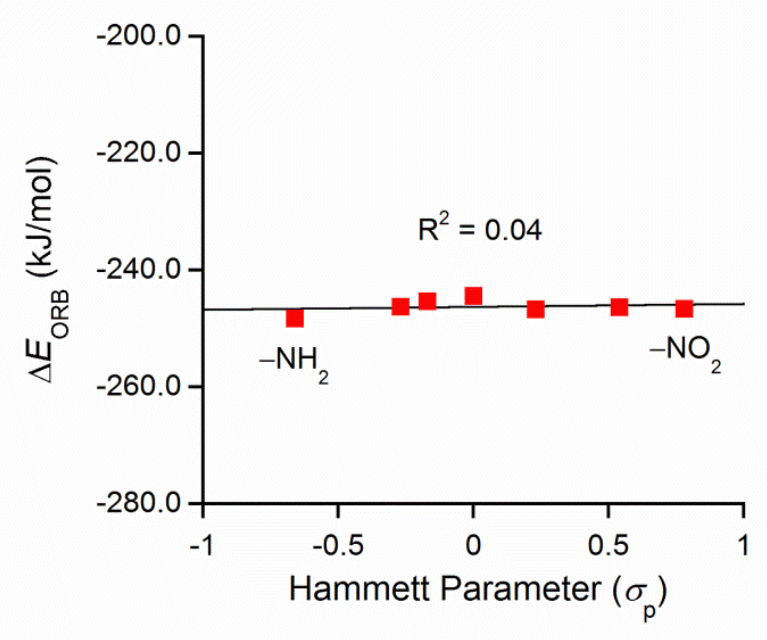

(d)

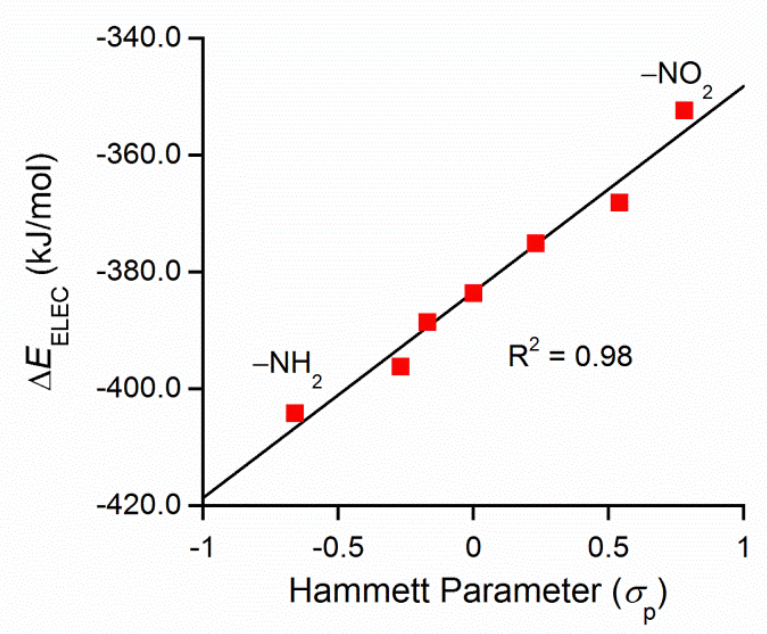

Figures S5a-d. EDA-NOCV calculated values of (a) $\Delta E_{\mathrm{BOND}}$, (b) $\Delta E_{\mathrm{ORB}}$, (c) $\Delta E_{\mathrm{PAULI}}$, and (d) $\Delta E_{\mathrm{ELEC}}$ versus Hammett parameter $\left(\sigma_{\mathrm{p}}\right)$ for $\left[(\mathrm{NHC}) \mathrm{Au}\left(\mathrm{X}-\mathrm{C}_{6} \mathrm{H}_{4}-\mathrm{CH}=\mathrm{CH}_{2}\right)\right]^{+}$complexes $\mathbf{3 a}-\mathbf{g}$. 
(a)

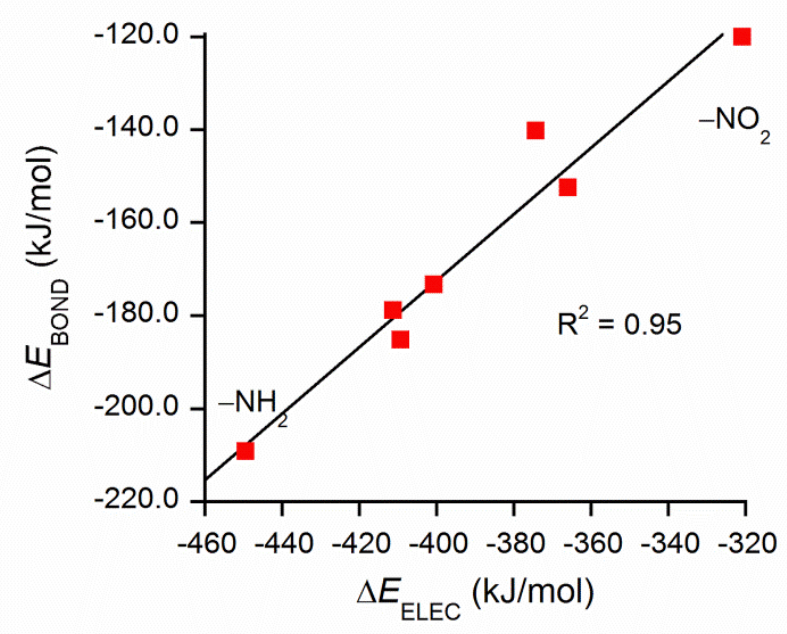

(b)

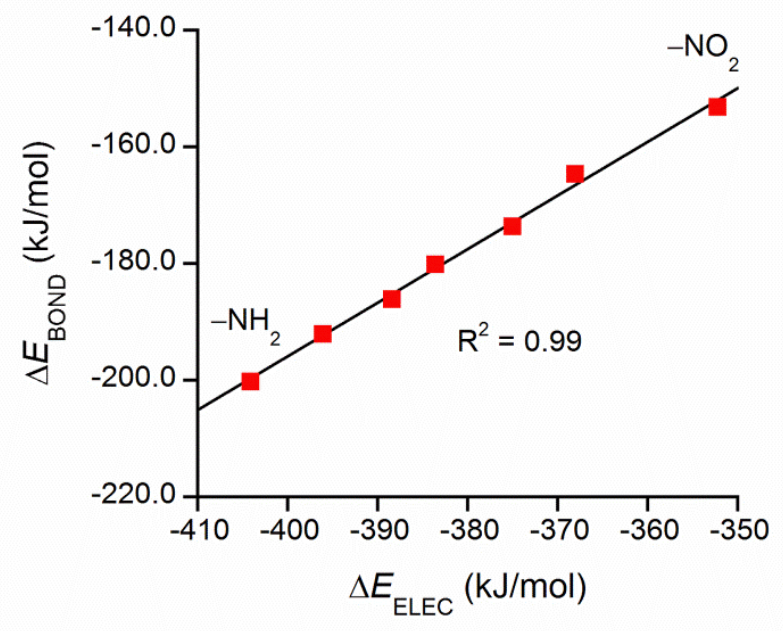

Figure S6a and S6b. EDA-NOCV calculated $\Delta E_{\mathrm{BOND}}$ versus $\Delta E_{\mathrm{ELEC}}$ for (a) [(NHC)Au(X$\left.\left.\mathrm{CH}=\mathrm{CH}_{2}\right)\right]^{+}$complexes $\mathbf{2 a}-\mathbf{g}$ and (b) $\left[(\mathrm{NHC}) \mathrm{Au}\left(\mathrm{X}-\mathrm{C}_{6} \mathrm{H}_{4}-\mathrm{CH}=\mathrm{CH}_{2}\right)\right]^{+}$complexes $\mathbf{3 a}-\mathbf{g}$. 
(a)

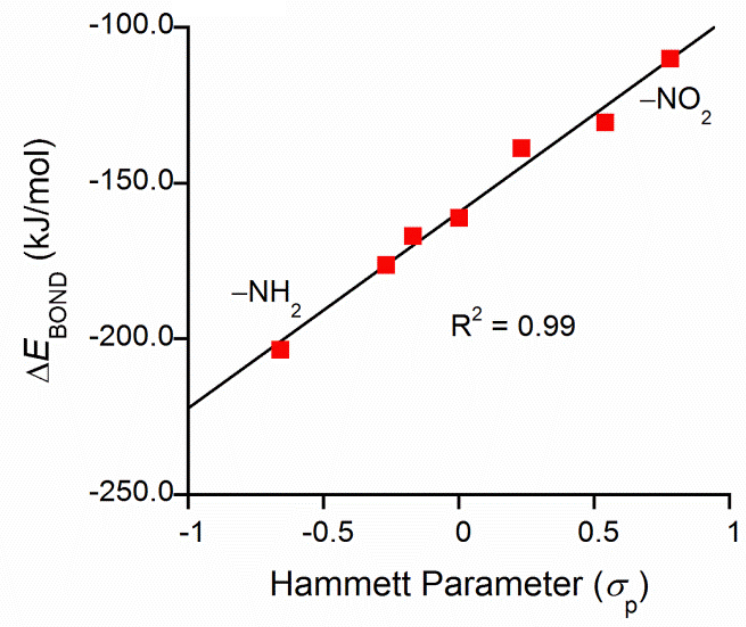

(c)

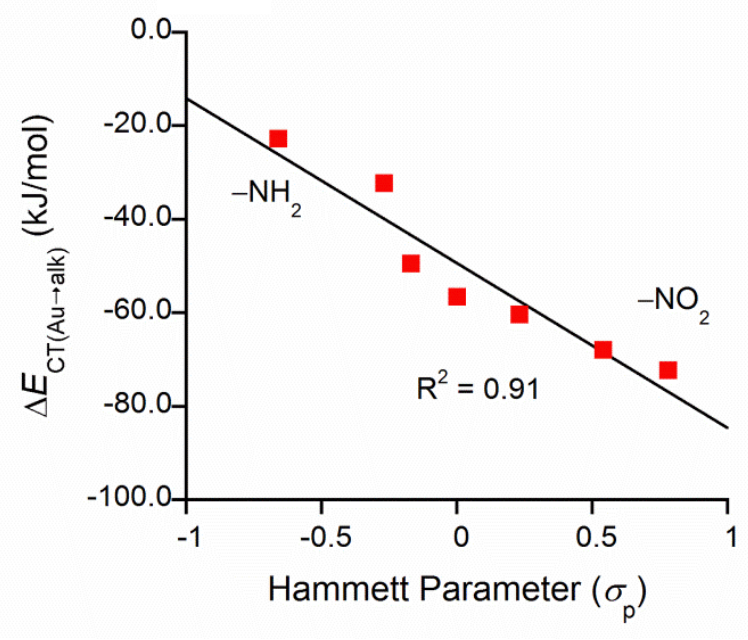

(b)

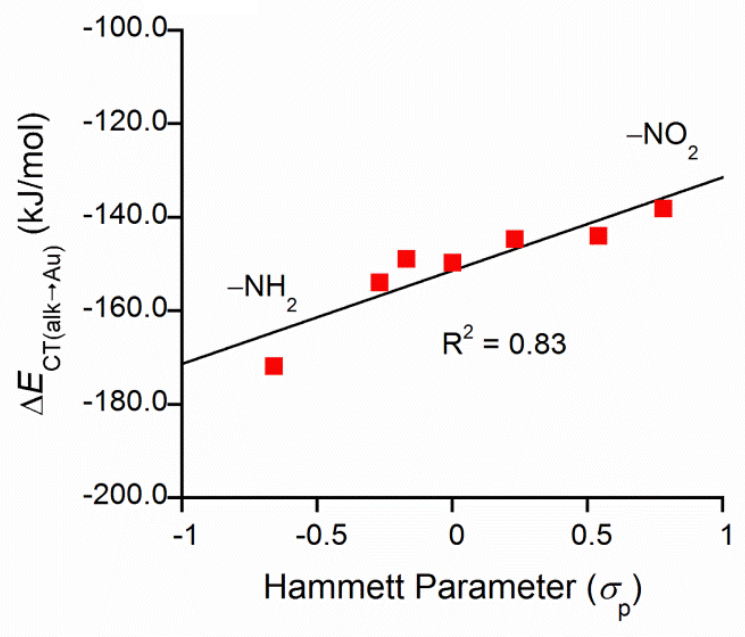

(d)

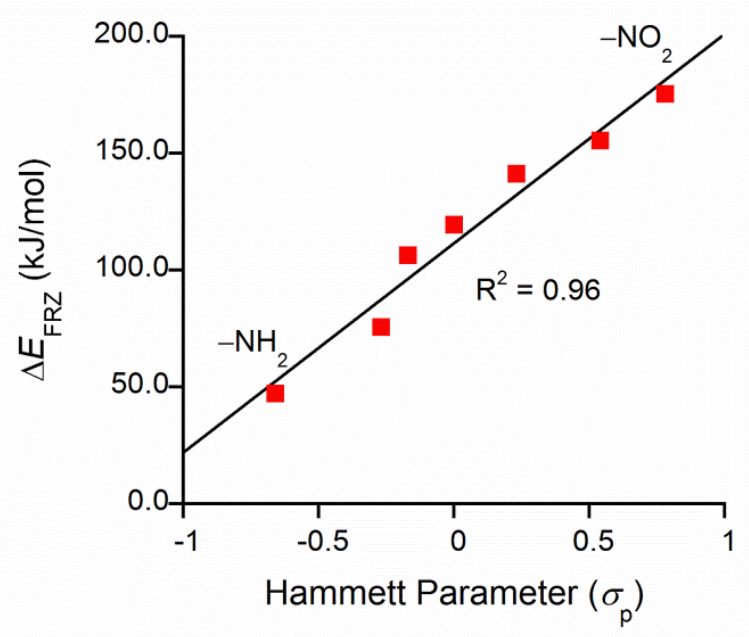

Figures S7a-d. ALMO-EDA calculated values of (a) $\Delta E_{\mathrm{BOND}}$, (b) $\Delta E_{\mathrm{CT}(\mathrm{alk} \rightarrow \mathrm{Au})}$, (c) $\Delta E_{\mathrm{CT}(\mathrm{Au} \rightarrow \mathrm{alk})}$, and (d) $\Delta E_{\mathrm{FRZ}}$ versus Hammett parameter $\left(\sigma_{\mathrm{p}}\right)$ for $\left[(\mathrm{NHC}) \mathrm{Au}\left(\mathrm{X}-\mathrm{CH}=\mathrm{CH}_{2}\right)\right]^{+}$complexes $\mathbf{2 a}-\mathbf{g}$. 
(a)

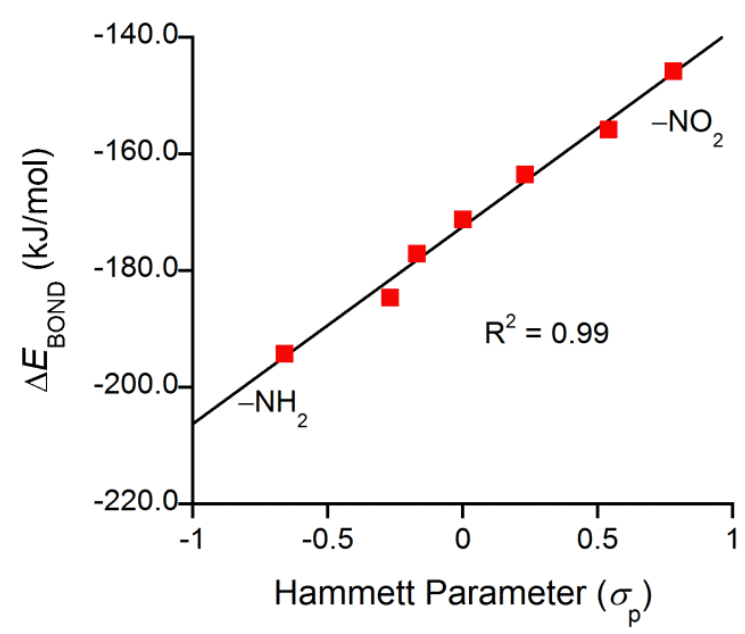

(c)

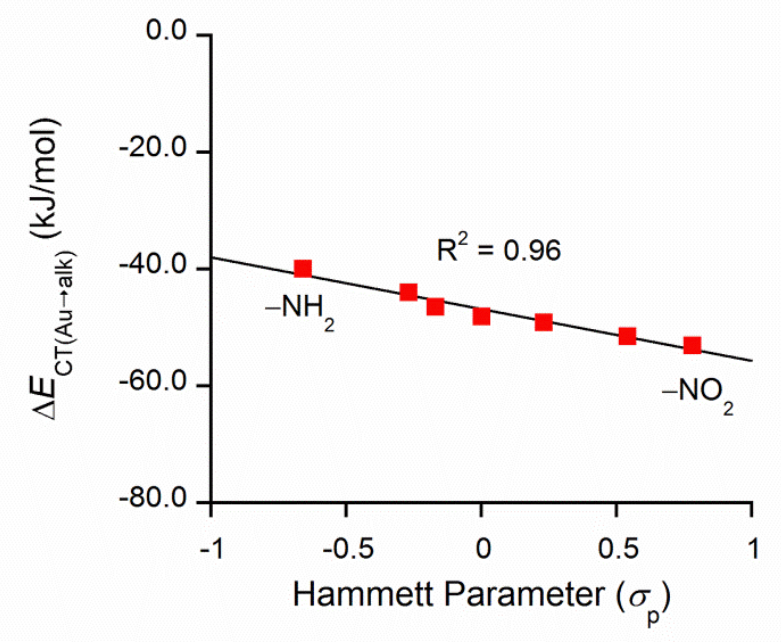

(b)

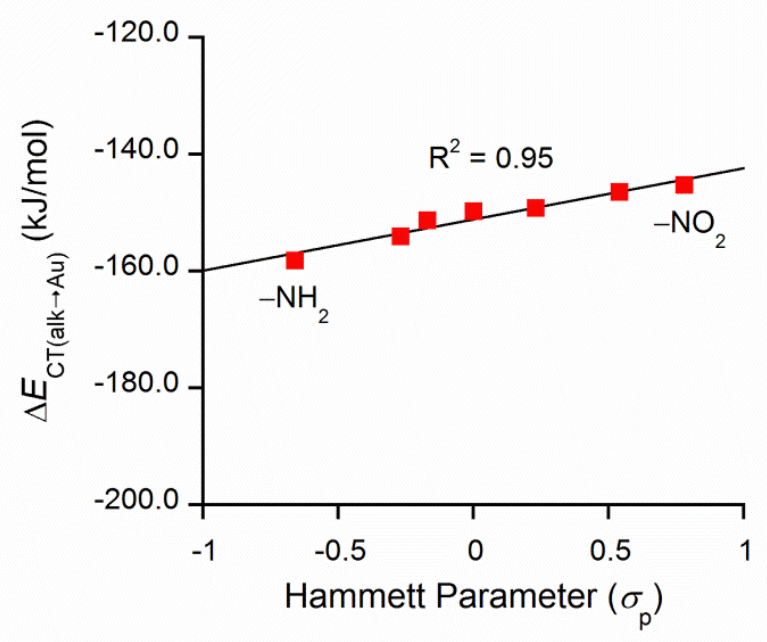

(d)

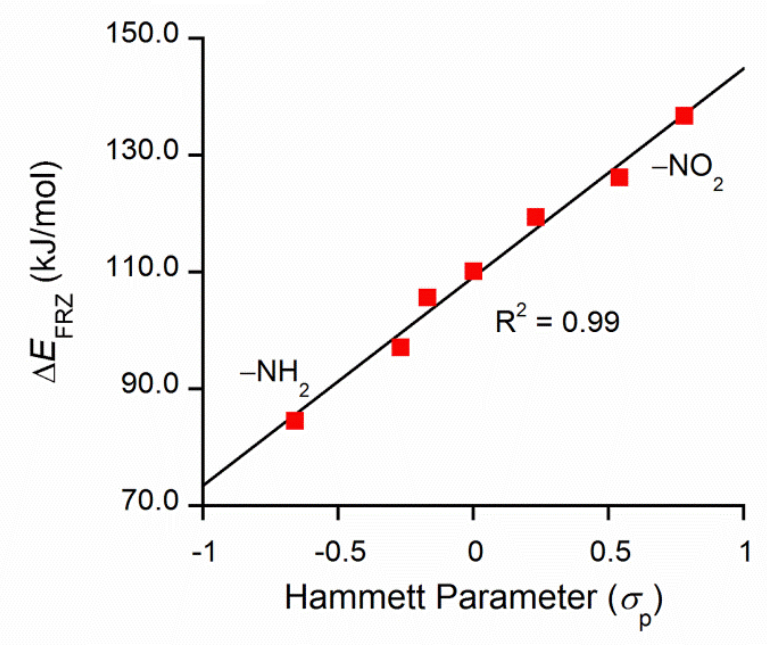

Figures S8a-d. ALMO-EDA calculated values of (a) $\Delta E_{\mathrm{BOND}}$, (b) $\Delta E_{\mathrm{CT}(\mathrm{alk} \rightarrow \mathrm{Au})}$, (c) $\Delta E_{\mathrm{CT}(\mathrm{Au} \rightarrow \mathrm{alk})}$, and (d) $\Delta E_{\mathrm{FRZ}}$ versus Hammett parameter $\left(\sigma_{\mathrm{p}}\right)$ for $\left[(\mathrm{NHC}) \mathrm{Au}\left(\mathrm{X}-\mathrm{C}_{6} \mathrm{H}_{4}-\mathrm{CH}=\mathrm{CH}_{2}\right)\right]^{+}$complexes 3a-g. 
(a)

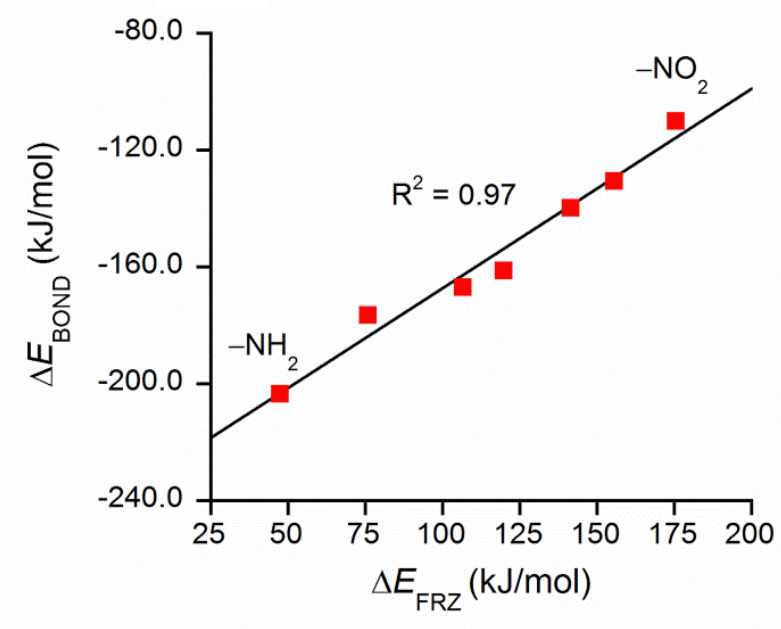

(b)

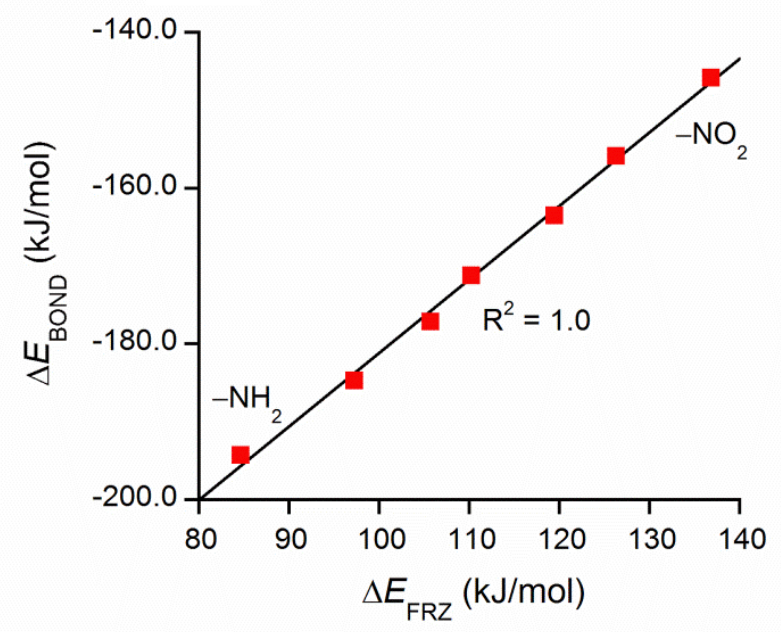

Figure S9a and S9b. ALMO-EDA calculated $\Delta E_{\mathrm{BOND}}$ versus $\Delta E_{\mathrm{FRZ}}$ for (a) [(NHC)Au(X$\left.\left.\mathrm{CH}=\mathrm{CH}_{2}\right)\right]^{+}$complexes $2 \mathbf{a}-\mathbf{g}$ and (b) $\left[(\mathrm{NHC}) \mathrm{Au}\left(\mathrm{X}-\mathrm{C}_{6} \mathrm{H}_{4}-\mathrm{CH}=\mathrm{CH}_{2}\right)\right]^{+}$complexes $\mathbf{3 a}-\mathbf{g}$. 


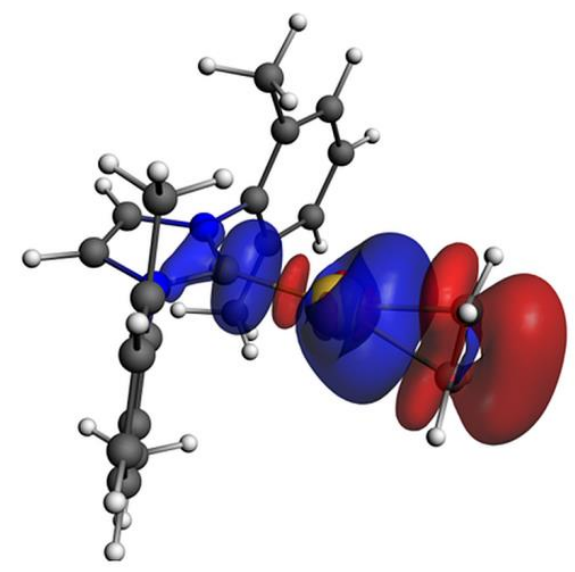

$\Delta \rho_{1}: \Delta \mathrm{E}=-126.6 \mathrm{~kJ} / \mathrm{mol} ; \mathrm{v}=0.4723$

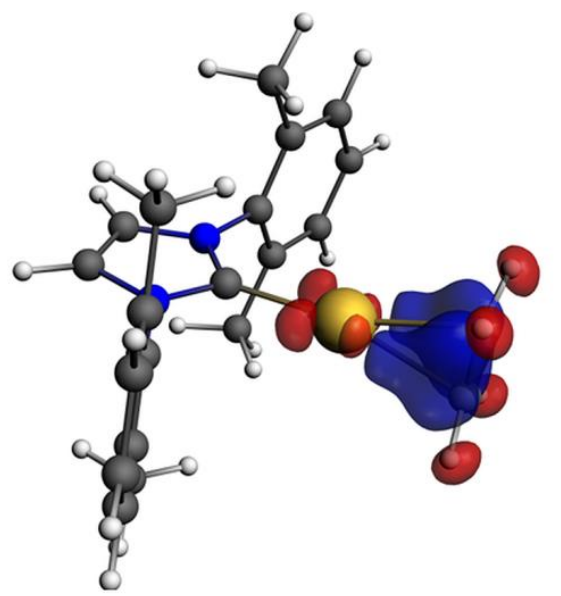

$\Delta \rho_{3}: \Delta \mathrm{E}=-12.8 \mathrm{~kJ} / \mathrm{mol} ; v=0.1064$

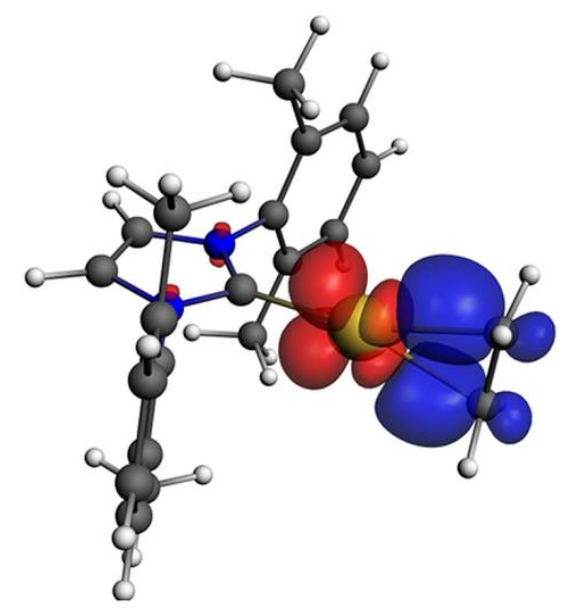

$\Delta \rho_{2}: \Delta \mathrm{E}=-73.4 \mathrm{~kJ} / \mathrm{mol} ; v=0.4077$

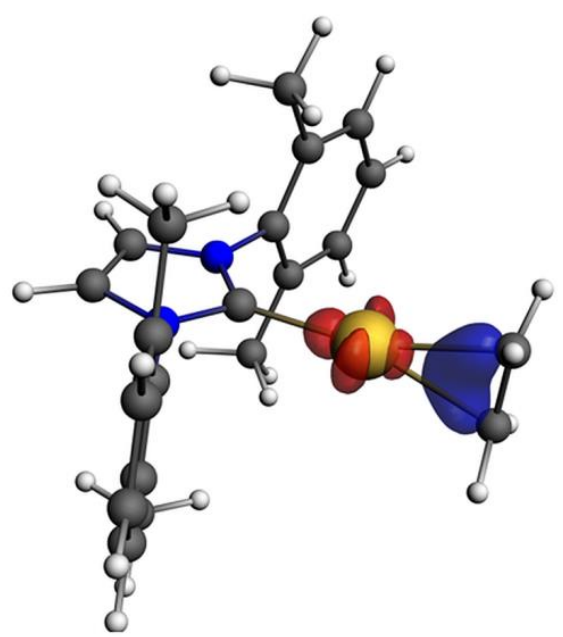

$\Delta \rho_{4}: \Delta \mathrm{E}=-10.1 \mathrm{~kJ} / \mathrm{mol} ; v=0.0943$

Figure S10. EDA-NOCV calculated deformation density plots $(\Delta \rho)$ associated with the four largest pairwise orbital interactions in 1d. The flow of electron charge is red $\rightarrow$ blue. Orbital interaction energies $(\Delta \mathrm{E})$ and eigenvalues $(v)$ are shown. 
Table S1. Supplementary geometric data for $\mathrm{Au}(\mathrm{I})$-alkene complexes (all bond angles in degrees).

\begin{tabular}{|c|c|c|c|}
\hline Complex $^{a}$ & $\angle \mathrm{C} 1-\mathrm{Au}-\mathrm{C} 2$ & $\angle \mathrm{Au}-\mathrm{C} 1-\mathrm{C} 2$ & Dihedral $\angle$ \\
\hline $1 \mathbf{a}$ & 35.5 & 72.2 & 90.0 \\
\hline $1 b$ & 35.6 & 72.2 & 90.0 \\
\hline $1 \mathrm{c}$ & 35.6 & 72.2 & 90.0 \\
\hline $1 d^{b}$ & 35.6 & 72.2 & 90.0 \\
\hline 1e & 35.6 & 72.2 & 90.0 \\
\hline 1f & 35.6 & 72.2 & 90.0 \\
\hline 19 & 35.7 & 72.2 & 89.2 \\
\hline $2 a$ & 35.7 & 71.3 & 93.7 \\
\hline $2 b$ & 35.8 & 71.6 & 99.0 \\
\hline $2 c$ & 35.6 & 73.1 & 99.5 \\
\hline $2 d^{b}$ & 35.6 & 72.2 & 90.0 \\
\hline $2 e$ & 35.4 & 75.2 & 85.0 \\
\hline $2 f$ & 33.5 & 86.0 & 74.5 \\
\hline $2 \mathrm{~g}$ & 31.3 & 95.7 & 57.6 \\
\hline $3 a$ & 35.5 & 75.0 & 86.6 \\
\hline $3 b$ & 35.5 & 74.7 & 88.1 \\
\hline $3 c$ & 35.4 & 76.0 & 89.8 \\
\hline 3d & 35.4 & 76.1 & 105.1 \\
\hline $3 e$ & 35.4 & 76.7 & 107.0 \\
\hline $3 f$ & 35.3 & 77.9 & 101.4 \\
\hline $3 g$ & 35.1 & 79.8 & 109.1 \\
\hline 4 & 37.3 & 71.3 & N/A \\
\hline
\end{tabular}

${ }^{a} \mathrm{X}=$ (a) $\mathrm{NO}_{2}$; (b) $\mathrm{CF}_{3}$; (c) $\mathrm{Br}$; (d) $\mathrm{H}$; (e) $\mathrm{CH}_{3}$; (f) $\mathrm{OCH}_{3}$; (g) $\mathrm{NH}_{2} .{ }^{b}$ Complex $\mathbf{1 d}$ and $\mathbf{2 d}$ are identical, but are listed throughout as separate complexes in the tables and figures for ease of comparison in each series. 
Table S2. Selected molecular orbital data for $\mathrm{Au}(\mathrm{I})$-alkene complexes.

\begin{tabular}{|c|c|c|c|}
\hline Complex & $\begin{array}{c}\% \text { Alkene } \\
\text { OFOs }^{a} \text { in } \\
\text { UMOs }^{c}\end{array}$ & $\begin{array}{l}\% \text { Alkene } \\
\text { UFOs }^{b} \text { in } \\
\text { OMOs }^{d}\end{array}$ & $\begin{array}{c}\text { Complex } \\
\text { LUMO Energy } \\
(\mathrm{eV})\end{array}$ \\
\hline $1 \mathrm{a}$ & 18.0 & 9.6 & -5.66 \\
\hline $1 b$ & 17.1 & 9.7 & -5.34 \\
\hline $1 c$ & 17.5 & 10.0 & -5.21 \\
\hline $1 d$ & 17.5 & 10.0 & -5.16 \\
\hline $1 e$ & 17.5 & 10.2 & -5.08 \\
\hline 1f & 16.9 & 10.3 & -5.03 \\
\hline $1 \mathrm{~g}$ & 17.5 & 10.6 & -4.98 \\
\hline $2 a$ & 16.4 & 12.4 & -6.26 \\
\hline $2 b$ & 18.8 & 11.6 & -5.53 \\
\hline $2 c$ & 16.4 & 10.5 & -5.26 \\
\hline $2 d$ & 17.5 & 10.0 & -5.16 \\
\hline $2 e$ & 18.2 & 9.1 & -4.87 \\
\hline $2 f$ & 21.6 & 6.9 & -4.56 \\
\hline $2 \mathrm{~g}$ & 22.3 & 5.5 & -4.31 \\
\hline $3 \mathbf{a}$ & 17.9 & 10.5 & -5.66 \\
\hline $3 b$ & 19.1 & 10.2 & -5.37 \\
\hline $3 c$ & 18.5 & 9.9 & -5.19 \\
\hline 3d & 18.4 & 9.5 & -5.06 \\
\hline $3 e$ & 18.9 & 9.4 & -4.95 \\
\hline 3f & 19.8 & 9.2 & -4.82 \\
\hline $3 g$ & 20.8 & 8.8 & -4.64 \\
\hline 4 & 27.5 & 11.5 & -7.19 \\
\hline
\end{tabular}

${ }^{a} \mathrm{OFO}=$ Occupied fragment orbital. ${ }^{b} \mathrm{UFO}=$ Unoccupied fragment orbital. ${ }^{c} \mathrm{OMO}=$ Occupied molecular orbital. ${ }^{d} \mathrm{UMO}=$ Unoccupied molecular orbital. 
Table S3. Energy of occupied and unoccupied molecular orbitals having the greatest contribution from the $\mathrm{C}_{2} \mathrm{H}_{4} \pi$-HOFO for [(X-NHC)Au( $\left.\left.\mathrm{C}_{2} \mathrm{H}_{4}\right)\right]^{+}$complexes $1 \mathrm{a}-\mathrm{g}$.

$\begin{array}{ccc}\text { Complex } & \begin{array}{c}\text { Energy }(\mathrm{eV}) \text { of } \mathrm{OMO}^{a} \\ \text { having greatest } \mathrm{C}_{2} \mathrm{H}_{4} \pi- \\ \text { HOFO contribution }\end{array} & \begin{array}{c}\text { Energy }(\mathrm{eV}) \text { of } \mathrm{UMO}^{b} \\ \text { having greatest } \mathrm{C}_{2} \mathrm{H}_{4} \pi- \\ \text { HOFO contribution }\end{array} \\ \mathbf{1 a} & -13.20 & -1.72 \\ \mathbf{1 b} & -13.04 & -1.59 \\ \mathbf{1 c} & -12.95 & -1.36 \\ \mathbf{1 d} & -12.86 & -1.49 \\ \mathbf{1 e} & -12.79 & -1.38 \\ \mathbf{1 f} & -12.75 & -1.36 \\ \mathbf{1 g} & -12.74 & -1.29\end{array}$

${ }^{a} \mathrm{OMO}=$ Occupied molecular orbital. ${ }^{b} \mathrm{UMO}=$ Unoccupied molecular orbital

Table S4. EDA-NOCV results for $\mathrm{Au}(\mathrm{I})$-alkene complexes (all energies in $\mathrm{kJ} / \mathrm{mol}$ ).

\begin{tabular}{ccccccccccc} 
Complex & $\Delta E_{\mathrm{PAULI}}$ & $\Delta E_{\mathrm{ELEC}}$ & $\Delta E_{\mathrm{ORB}}$ & $\Delta E_{\mathrm{ORB}(1)}{ }^{a}$ & $\Delta E_{\mathrm{ORB}(2)}{ }^{a}$ & $\Delta E_{\mathrm{ORB}(3)}{ }^{a}$ & $\Delta E_{\mathrm{ORB}(4)}{ }^{a}$ & $\Delta E_{\mathrm{ORB}(\mathrm{rest})}{ }^{a}$ & $\Delta E_{\mathrm{PREP}}$ & $\Delta E_{\mathrm{BOND}}$ \\
$\mathbf{1 a}$ & 441.8 & -397.1 & -239.7 & -129.3 & -70.4 & -12.9 & -10.2 & -17.3 & 15.9 & -179.2 \\
$\mathbf{1 b}$ & 444.6 & -398.4 & -239.2 & -128.0 & -71.7 & -12.9 & -10.1 & -17.2 & 15.5 & -177.6 \\
$\mathbf{1 c}$ & 450.4 & -401.9 & -240.0 & -127.1 & -73.6 & -12.8 & -10.2 & -17.0 & 18.6 & -173.0 \\
$\mathbf{1 d}$ & 449.5 & -400.8 & -239.1 & -126.6 & -73.4 & -12.8 & -10.1 & -16.9 & 17.3 & -173.2 \\
$\mathbf{1 e}$ & 452.8 & -402.3 & -239.5 & -125.9 & -74.4 & -12.7 & -10.1 & -16.9 & 17.6 & -171.4 \\
$\mathbf{1 f}$ & 455.3 & -404.5 & -240.6 & -125.8 & -75.3 & -12.8 & -10.2 & -17.0 & 16.0 & -173.7 \\
$\mathbf{1 g}$ & 459.8 & -407.4 & -242.4 & -126.0 & -76.9 & -12.8 & -10.2 & -17.0 & 16.6 & -173.5 \\
\hline $\mathbf{2 a}$ & 433.6 & -321.0 & -253.7 & -96.8 & -112.6 & -14.9 & -10.4 & -19.3 & 21.3 & -119.8 \\
$\mathbf{2 b}$ & 462.9 & -374.4 & -251.6 & -112.5 & -94.2 & -14.3 & -10.5 & -20.1 & 22.8 & -140.3 \\
$\mathbf{2 c}$ & 440.3 & -366.1 & -247.5 & -118.8 & -79.3 & -14.6 & -8.9 & -24.7 & 20.2 & -153.1 \\
$\mathbf{2 d}$ & 449.5 & -400.8 & -239.1 & -126.6 & -73.4 & -12.8 & -10.1 & -16.9 & 17.3 & -173.2 \\
$\mathbf{2 e}$ & 448.4 & -411.4 & -235.4 & -127.9 & -65.4 & -12.3 & -9.7 & -20.7 & 19.6 & -178.8 \\
$\mathbf{2 f}$ & 421.7 & -409.4 & -223.7 & -142.6 & -40.9 & -11.4 & -8.8 & -20.9 & 26.3 & -185.1 \\
$\mathbf{2 g}$ & 430.3 & -449.6 & -230.3 & -160.8 & -32.8 & -10.7 & $\mathrm{~N} / \mathrm{A}$ & -26.8 & 40.6 & -209.1 \\
\hline $\mathbf{3 a}$ & 427.0 & -352.3 & -246.6 & -125.9 & -72.9 & -10.9 & -11.0 & -26.5 & 18.8 & -153.1 \\
$\mathbf{3 b}$ & 432.1 & -368.1 & -246.4 & -125.8 & -69.7 & -11.0 & -10.8 & -29.5 & 17.8 & -164.6 \\
$\mathbf{3 c}$ & 428.7 & -375.0 & -246.7 & -129.5 & -68.2 & -9.4 & -10.5 & -29.5 & 19.4 & -173.6 \\
$\mathbf{3 d}$ & 428.6 & -383.6 & -244.4 & -129.8 & -66.9 & -9.8 & -9.4 & -29.2 & 19.4 & -180.1 \\
$\mathbf{3 e}$ & 428.2 & -388.5 & -245.3 & -131.8 & -65.2 & -8.6 & -9.0 & -31.7 & 19.5 & -186.0 \\
$\mathbf{3 f}$ & 428.4 & -396.1 & -246.2 & -135.2 & -62.3 & -10.2 & -10.2 & -29.3 & 21.9 & -192.0 \\
$\mathbf{3 g}$ & 425.2 & -404.2 & -248.2 & -141.4 & -57.6 & -10.6 & -10.0 & -28.8 & 27.1 & -200.2 \\
\hline $\mathbf{4}$ & 595.3 & -528.7 & -368.7 & -211.0 & -86.0 & -25.3 & -19.7 & -9.1 & 25.2 & -276.8
\end{tabular}

${ }^{a}$ The four largest components of $\Delta E_{\mathrm{ORB}}$ are given individually, with the sum of the remaining components given as $\Delta E_{\mathrm{ORB}(\text { rest) }}$. 
Table S5. ALMO energy decomposition analysis results for Au(I)-alkene complexes (energies in $\mathrm{kJ} / \mathrm{mol}$ ).

\begin{tabular}{cccccccc} 
Complex & $\Delta E_{\mathrm{FRZ}}$ & $\Delta E_{\mathrm{POL}}$ & $\Delta E_{\mathrm{CT}(\mathrm{alkne} \rightarrow \mathrm{Au})}$ & $\Delta E_{\mathrm{CT}(\mathrm{Au} \rightarrow \mathrm{alkene})}$ & $\Delta E_{\mathrm{CT}(\mathrm{HO})}$ & $\Delta E_{\mathrm{PREP}}$ & $\Delta E_{\mathrm{BOND}}$ \\
$\mathbf{1 a}$ & 114.5 & -76.2 & -151.1 & -53.7 & -16.6 & 15.9 & -167.2 \\
$\mathbf{1 b}$ & 118.1 & -75.6 & -150.4 & -54.8 & -16.8 & 15.5 & -164.0 \\
$\mathbf{1 c}$ & 118.1 & -76.4 & -150.2 & -52.9 & -16.8 & 18.6 & -159.7 \\
$\mathbf{1 d}$ & 119.6 & -74.8 & -149.7 & -56.5 & -17.0 & 17.3 & -161.1 \\
$\mathbf{1 e}$ & 120.4 & -74.5 & -149.3 & -57.5 & -17.2 & 17.6 & -160.6 \\
$\mathbf{1 f}$ & 122.0 & -74.6 & -149.9 & -58.4 & -17.4 & 16.0 & -162.2 \\
$\mathbf{1 g}$ & 125.3 & -74.7 & -150.2 & -60.0 & -17.7 & 16.6 & -160.8 \\
\hline $\mathbf{2 a}$ & 175.4 & -77.4 & -138.0 & -72.2 & -18.8 & 21.3 & -109.8 \\
$\mathbf{2 b}$ & 155.4 & -78.5 & -143.9 & -67.9 & -18.5 & 22.8 & -130.6 \\
$\mathbf{2 c}$ & 141.4 & -78.4 & -145.5 & -60.3 & -17.8 & 20.2 & -139.5 \\
$\mathbf{2 d}$ & 119.6 & -74.8 & -149.7 & -56.5 & -17.0 & 17.3 & -161.1 \\
$\mathbf{2 e}$ & 106.5 & -78.3 & -148.9 & -49.5 & -16.2 & 19.6 & -166.8 \\
$\mathbf{2 f}$ & 75.7 & -79.8 & -153.8 & -32.2 & -12.6 & 26.3 & -176.3 \\
$\mathbf{2 g}$ & 47.3 & -86.9 & -171.8 & -22.7 & -9.9 & 40.6 & -203.3 \\
\hline 3a & 136.8 & -86.3 & -145.2 & -53.0 & -16.8 & 18.8 & -145.8 \\
$\mathbf{3 b}$ & 126.3 & -85.3 & -146.4 & -51.4 & -16.7 & 17.8 & -155.8 \\
$\mathbf{3 c}$ & 119.4 & -87.7 & -149.1 & -49.1 & -16.4 & 19.4 & -163.5 \\
$\mathbf{3 d}$ & 110.2 & -86.8 & -149.7 & -48.1 & -16.1 & 19.4 & -171.2 \\
$\mathbf{3 e}$ & 105.7 & -88.5 & -151.3 & -46.4 & -16.1 & 19.5 & -177.1 \\
$\mathbf{3 f}$ & 97.2 & -89.9 & -154.0 & -43.9 & -15.9 & 21.9 & -184.6 \\
$\mathbf{3 g}$ & 84.6 & -92.4 & -158.2 & -39.9 & -15.4 & 27.1 & -194.2 \\
\hline $\mathbf{4}$ & 162.6 & -120.7 & -230.3 & -60.7 & -27.8 & 25.2 & -251.7
\end{tabular}

Table S6. ETS energy decomposition analysis results for $(\mathrm{X}-\mathrm{NHC}) \mathrm{AuCl}$ complexes (energies in $\mathrm{kJ} / \mathrm{mol}$ ).

\begin{tabular}{|c|c|c|c|c|c|}
\hline Complex & $\Delta E_{\mathrm{PAULI}}$ & $\Delta E_{\mathrm{ELEC}}$ & $\Delta E_{\mathrm{ORB}}$ & $\Delta E_{\mathrm{PREP}}$ & $\Delta E_{\mathrm{BOND}}$ \\
\hline (NO & 427. & -79 & -28 & 6. & -6 \\
\hline$\left(\mathrm{NH}_{2}-\mathrm{NHC}\right) \mathrm{AuCl}$ & 416.1 & -754.2 & -269.5 & 4.2 & -603.4 \\
\hline
\end{tabular}




\section{Optimized Atomic coordinates $(\AA)$ \\ Energies (au) calculated at the PBE0 level of theory with the SDD basis set and ECP used for Au and the 6-31g(d,p) basis set for all other atoms.}

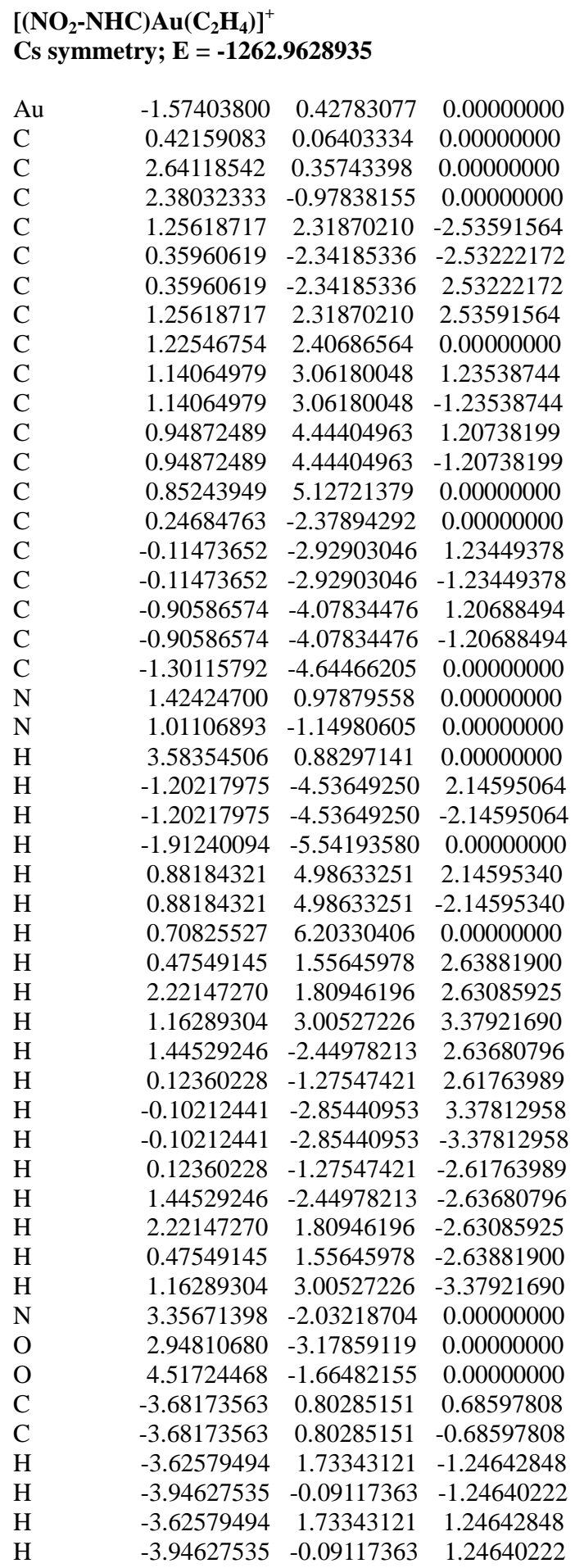


$\left[\left(\mathrm{CF}_{3}-\mathrm{NHC}\right) \mathrm{Au}\left(\mathrm{C}_{2} \mathrm{H}_{4}\right)\right]^{+}$

Cs symmetry; $\mathrm{E}=\mathbf{- 1 3 9 5 . 4 0 6 3 1 7 7}$

\begin{tabular}{|c|c|c|c|}
\hline $\mathrm{Au}$ & -1.60016811 & 0.83494303 & 0.00000000 \\
\hline $\mathrm{C}$ & 0.29463187 & 0.11514966 & 0.00000000 \\
\hline $\mathrm{C}$ & 2.52906337 & -0.00863765 & 0.00000000 \\
\hline $\mathrm{C}$ & 2.04255222 & -1.27775762 & 0.00000000 \\
\hline $\mathrm{C}$ & 1.52200190 & 2.17847110 & -2.53500172 \\
\hline $\mathrm{C}$ & -0.21334042 & -2.21658070 & -2.53713254 \\
\hline $\mathrm{C}$ & -0.21334042 & -2.21658070 & 2.53713254 \\
\hline $\mathrm{C}$ & 1.52200190 & 2.17847110 & 2.53500172 \\
\hline $\mathrm{C}$ & 1.51069679 & 2.26918632 & 0.00000000 \\
\hline $\mathrm{C}$ & 1.54956020 & 2.93015355 & 1.23457111 \\
\hline $\mathrm{C}$ & 1.54956020 & 2.93015355 & -1.23457111 \\
\hline $\mathrm{C}$ & 1.61888805 & 4.32401032 & 1.20716078 \\
\hline $\mathrm{C}$ & 1.61888805 & 4.32401032 & -1.20716078 \\
\hline $\mathrm{C}$ & 1.65179186 & 5.01347969 & 0.00000000 \\
\hline $\mathrm{C}$ & -0.27218993 & -2.28120555 & 0.00000000 \\
\hline $\mathrm{C}$ & -0.70884536 & -2.77810089 & 1.23530513 \\
\hline $\mathrm{C}$ & -0.70884536 & -2.77810089 & -1.23530513 \\
\hline $\mathrm{C}$ & -1.62732916 & -3.82867751 & 1.20694610 \\
\hline $\mathrm{C}$ & -1.62732916 & -3.82867751 & -1.20694610 \\
\hline $\mathrm{C}$ & -2.08062089 & -4.34926802 & 0.00000000 \\
\hline $\mathrm{N}$ & 1.44212332 & 0.83041536 & 0.00000000 \\
\hline $\mathrm{N}$ & 0.66433923 & -1.18307549 & 0.00000000 \\
\hline $\mathrm{H}$ & 3.54479868 & 0.35541917 & 0.00000000 \\
\hline $\mathrm{H}$ & -1.98299455 & -4.24285632 & 2.14581327 \\
\hline $\mathrm{H}$ & -1.98299455 & -4.24285632 & -2.14581327 \\
\hline $\mathrm{H}$ & -2.79101416 & -5.17038654 & 0.00000000 \\
\hline $\mathrm{H}$ & 1.65418775 & 4.86909458 & 2.14589559 \\
\hline $\mathrm{H}$ & 1.65418775 & 4.86909458 & -2.14589559 \\
\hline $\mathrm{H}$ & 1.71097453 & 6.09760673 & 0.00000000 \\
\hline $\mathrm{H}$ & 0.60370535 & 1.59025477 & 2.64338018 \\
\hline $\mathrm{H}$ & 2.36322026 & 1.48236384 & 2.62180264 \\
\hline $\mathrm{H}$ & 1.57642981 & 2.86896699 & 3.37859357 \\
\hline $\mathrm{H}$ & 0.86526798 & -2.36305286 & 2.64875718 \\
\hline $\mathrm{H}$ & -0.41050362 & -1.14165896 & 2.61795933 \\
\hline $\mathrm{H}$ & -0.70456054 & -2.70943119 & 3.37814932 \\
\hline $\mathrm{H}$ & -0.70456054 & -2.70943119 & -3.37814932 \\
\hline $\mathrm{H}$ & -0.41050362 & -1.14165896 & -2.61795933 \\
\hline $\mathrm{H}$ & 0.86526798 & -2.36305286 & -2.64875718 \\
\hline $\mathrm{H}$ & 2.36322026 & 1.48236384 & -2.62180264 \\
\hline $\mathrm{H}$ & 0.60370535 & 1.59025477 & -2.64338018 \\
\hline $\mathrm{H}$ & 1.57642981 & 2.86896699 & -3.37859357 \\
\hline $\mathrm{C}$ & 2.81230759 & -2.55969916 & 0.00000000 \\
\hline $\mathrm{F}$ & 4.11678274 & -2.28197555 & 0.00000000 \\
\hline $\mathrm{F}$ & 2.53514616 & -3.29950960 & -1.07872612 \\
\hline $\mathrm{F}$ & 2.53514616 & -3.29950960 & 1.07872612 \\
\hline $\mathrm{C}$ & -3.60198627 & 1.59113584 & 0.68601511 \\
\hline $\mathrm{C}$ & -3.60198627 & 1.59113584 & -0.68601511 \\
\hline $\mathrm{H}$ & -3.37535539 & 2.49538873 & -1.24647638 \\
\hline $\mathrm{H}$ & -4.02798513 & 0.76193455 & -1.24650994 \\
\hline $\mathrm{H}$ & -3.37535539 & 2.49538873 & 1.24647638 \\
\hline $\mathrm{H}$ & -4.02798513 & 0.76193455 & 1.24650994 \\
\hline
\end{tabular}


$\left[(\mathrm{Br}-\mathrm{NHC}) \mathrm{Au}\left(\mathrm{C}_{2} \mathrm{H}_{4}\right)\right]^{+}$

Cs symmetry; $\mathrm{E}=\mathbf{- 3 6 2 9 . 1 5 6 9 8 8 1}$

$\begin{array}{lrrc}\mathrm{Au} & 1.58533793 & -0.92576032 & 0.00000000 \\ \mathrm{C} & -0.27823555 & -0.13221404 & 0.00000000 \\ \mathrm{C} & -2.51061263 & 0.07971080 & 0.00000000 \\ \mathrm{C} & -1.97269138 & 1.32970039 & 0.00000000 \\ \mathrm{C} & -1.58298434 & -2.14275494 & -2.53460906 \\ \mathrm{C} & 0.27013168 & 2.21268823 & -2.53188073 \\ \mathrm{C} & 0.27013168 & 2.21268823 & 2.53188073 \\ \mathrm{C} & -1.58298434 & -2.14275494 & 2.53460906 \\ \mathrm{C} & -1.57653451 & -2.23330970 & 0.00000000 \\ \mathrm{C} & -1.64266935 & -2.89270852 & 1.23432067 \\ \mathrm{C} & -1.64266935 & -2.89270852 & -1.23432067 \\ \mathrm{C} & -1.76792336 & -4.28266647 & 1.20709113 \\ \mathrm{C} & -1.76792336 & -4.28266647 & -1.20709113 \\ \mathrm{C} & -1.82843723 & -4.97037630 & 0.00000000 \\ \mathrm{C} & 0.36520223 & 2.25169080 & 0.00000000 \\ \mathrm{C} & 0.80700778 & 2.74464413 & 1.23432497 \\ \mathrm{C} & 0.80700778 & 2.74464413 & -1.23432497 \\ \mathrm{C} & 1.75544909 & 3.76814462 & 1.20734207 \\ \mathrm{C} & 1.75544909 & 3.76814462 & -1.20734207 \\ \mathrm{C} & 2.22695724 & 4.27209387 & 0.00000000 \\ \mathrm{~N} & -1.45088443 & -0.79922859 & 0.00000000 \\ \mathrm{~N} & -0.59881658 & 1.18304424 & 0.00000000 \\ \mathrm{H} & -3.53876366 & -0.24549055 & 0.00000000 \\ \mathrm{H} & 2.11843889 & 4.17624636 & 2.14613494 \\ \mathrm{H} & 2.11843889 & 4.17624636 & -2.14613494 \\ \mathrm{H} & 2.96201200 & 5.07125058 & 0.00000000 \\ \mathrm{H} & -1.82529934 & -4.82579817 & 2.14590214 \\ \mathrm{H} & -1.82529934 & -4.82579817 & -2.14590214 \\ \mathrm{H} & -1.93138750 & -6.05124678 & 0.00000000 \\ \mathrm{H} & -0.63095613 & -1.61253082 & 2.65114213 \\ \mathrm{H} & -2.37969201 & -1.39522609 & 2.61124498 \\ \mathrm{H} & -1.68829090 & -2.82758324 & 3.37804810 \\ \mathrm{H} & -0.80616072 & 2.39763598 & 2.62277826 \\ \mathrm{H} & 0.42446841 & 1.13204172 & 2.62546654 \\ \mathrm{H} & 3.29011499 & -2.65609234 & 1.24660354 \\ \mathrm{H} & 0.76146203 & 2.69581860 & 3.37850376 \\ \mathrm{H} & 0.76146203 & 2.69581860 & -3.37850376 \\ \mathrm{H} & 0.42446841 & 1.13204172 & -2.62546654 \\ \mathrm{H} & -0.80616072 & 2.39763598 & -2.62277826 \\ \mathrm{H} & -2.37969201 & -1.39522609 & -2.61124498 \\ \mathrm{H} & -0.63095613 & -1.61253082 & -2.65114213 \\ \mathrm{H} & -1.68829090 & -2.82758324 & -3.37804810 \\ \mathrm{H} & -2.80550674 & 2.97354347 & 0.00000000 \\ \mathrm{H} & 3.55254068 & -1.76156915 & 0.68622318 \\ \mathrm{H} & & -0.95115643 & 1.24667947\end{array}$


$\left[(\mathrm{H}-\mathrm{NHC}) \mathrm{Au}\left(\mathrm{C}_{2} \mathrm{H}_{4}\right)\right]^{+}$(perpendicular geometry) C2v symmetry; $\mathrm{E}=-\mathbf{1 0 5 8 . 6 8 6 2 0 6 4}$

$\begin{array}{lrrr}\mathrm{Au} & 0.00000000 & 0.00000000 & 1.30255117 \\ \mathrm{C} & 0.00000000 & 0.00000000 & -0.72264128 \\ \mathrm{C} & 0.00000000 & 0.67943175 & -2.85862829 \\ \mathrm{C} & -0.00000000 & -0.67943175 & -2.85862829 \\ \mathrm{C} & -2.53411595 & 2.36352241 & -1.12964986 \\ \mathrm{C} & -2.53411595 & -2.36352241 & -1.12964986 \\ \mathrm{C} & 2.53411595 & -2.36352241 & -1.12964986 \\ \mathrm{C} & 2.53411595 & 2.36352241 & -1.12964986 \\ \mathrm{C} & 0.00000000 & 2.44367600 & -1.09016552 \\ \mathrm{C} & 1.23375469 & 3.07672527 & -0.89051578 \\ \mathrm{C} & -1.23375469 & 3.07672527 & -0.89051578 \\ \mathrm{C} & 1.20688998 & 4.40414433 & -0.45949448 \\ \mathrm{C} & -1.20688998 & 4.40414433 & -0.45949448 \\ \mathrm{C} & 0.00000000 & 5.06060667 & -0.24495557 \\ \mathrm{C} & -0.00000000 & -2.44367600 & -1.09016552 \\ \mathrm{C} & 1.23375469 & -3.07672527 & -0.89051578 \\ \mathrm{C} & -1.23375469 & -3.07672527 & -0.89051578 \\ \mathrm{C} & 1.20688998 & -4.40414433 & -0.45949448 \\ \mathrm{C} & -1.20688998 & -4.40414433 & -0.45949448 \\ \mathrm{C} & -0.00000000 & -5.06060667 & -0.24495557 \\ \mathrm{~N} & 0.00000000 & 1.07628814 & -1.53905405 \\ \mathrm{~N} & -0.00000000 & -1.07628814 & -1.53905405 \\ \mathrm{H} & 0.00000000 & 1.39190913 & -3.66891619 \\ \mathrm{H} & -0.00000000 & -1.39190913 & -3.66891619 \\ \mathrm{H} & 2.14582320 & -4.92585522 & -0.29843548 \\ \mathrm{H} & -2.14582320 & -4.92585522 & -0.29843548 \\ \mathrm{H} & 0.00000000 & -6.09491988 & 0.08534038 \\ \mathrm{H} & 2.14582320 & 4.92585522 & -0.29843548 \\ \mathrm{H} & -2.14582320 & 4.92585522 & -0.29843548 \\ \mathrm{H} & 0.00000000 & 6.09491988 & 0.08534038 \\ \mathrm{H} & 2.64622284 & 1.49668720 & -0.46856418 \\ \mathrm{H} & 2.61412624 & 1.99615018 & -2.15827273 \\ \mathrm{H} & 3.37789317 & 3.03213847 & -0.94921802 \\ \mathrm{H} & 2.61412624 & -1.99615018 & -2.15827273 \\ \mathrm{H} & 2.64622284 & -1.49668720 & -0.46856418 \\ \mathrm{H} & 3.37789317 & -3.03213847 & -0.94921802 \\ \mathrm{H} & -3.37789317 & -3.03213847 & -0.94921802 \\ \mathrm{H} & -2.64622284 & -1.49668720 & -0.46856418 \\ \mathrm{H} & -2.61412624 & -1.99615018 & -2.15827273 \\ \mathrm{H} & -2.61412624 & 1.99615018 & -2.15827273 \\ \mathrm{H} & -2.64622284 & 1.49668720 & -0.46856418 \\ \mathrm{H} & -3.37789317 & 3.03213847 & -0.94921802 \\ \mathrm{H} & 0.68612462 & -0.00000000 & 3.44080285 \\ -0.68612462 & 0.00000000 & 3.44080285 \\ \mathrm{H} & -1.24660332 & 0.92593105 & 3.54834419 \\ \mathrm{H} & 1.24660332 & -0.92593105 & 3.54834419 \\ \mathrm{H} & & 0.92593105 & 3.54834419 \\ \mathrm{H} & & -0.92593105 & 3.54834419\end{array}$


$\left[(\mathrm{H}-\mathrm{NHC}) \mathrm{Au}\left(\mathrm{C}_{2} \mathrm{H}_{4}\right)\right]^{+}$(planar geometry; transition state structure) C2v symmetry; $E=-1058.6851865 ; 1$ imaginary frequency: $-29.8201 \mathrm{~cm}^{-1}$

\begin{tabular}{|c|c|c|c|}
\hline $\mathrm{Au}$ & -0.00000000 & 0.00000000 & 1.29821373 \\
\hline $\mathrm{C}$ & 0.00000000 & -0.00000000 & -0.73196967 \\
\hline $\mathrm{C}$ & 0.00000000 & 0.67851768 & -2.86801192 \\
\hline $\mathrm{C}$ & -0.00000000 & -0.67851768 & -2.86801192 \\
\hline $\mathrm{H}$ & 0.00000000 & 1.39197617 & -3.67733806 \\
\hline $\mathrm{H}$ & -0.00000000 & -1.39197617 & -3.67733806 \\
\hline $\mathrm{N}$ & 0.00000000 & 1.07590856 & -1.54560082 \\
\hline $\mathrm{N}$ & -0.00000000 & -1.07590856 & -1.54560082 \\
\hline $\mathrm{C}$ & 0.00000000 & 0.68534275 & 3.44427308 \\
\hline $\mathrm{C}$ & -0.00000000 & -0.68534275 & 3.44427308 \\
\hline $\mathrm{H}$ & -0.92541178 & -1.24751394 & 3.54724191 \\
\hline $\mathrm{H}$ & 0.92541178 & -1.24751394 & 3.54724191 \\
\hline $\mathrm{C}$ & -0.00000000 & -2.44097443 & -1.09082463 \\
\hline $\mathrm{C}$ & 1.23379319 & -3.07307698 & -0.88688794 \\
\hline $\mathrm{C}$ & -1.23379319 & -3.07307698 & -0.8868879 \\
\hline $\mathrm{C}$ & 1.20699722 & -4.39761390 & -0.44701235 \\
\hline $\mathrm{C}$ & -1.20699722 & -4.39761390 & -0.44701235 \\
\hline $\mathrm{C}$ & -0.00000000 & -5.05259123 & -0.22813920 \\
\hline $\mathrm{H}$ & 2.14590405 & -4.91846556 & -0.28295166 \\
\hline $\mathrm{H}$ & -2.14590405 & -4.91846556 & -0.28295166 \\
\hline $\mathrm{H}$ & -0.00000000 & -6.08483863 & 0.10863342 \\
\hline $\mathrm{C}$ & 0.00000000 & 2.44097443 & -1.09082463 \\
\hline $\mathrm{C}$ & 1.23379319 & 3.07307698 & -0.88688794 \\
\hline $\mathrm{C}$ & -1.23379319 & 3.07307698 & -0.88688794 \\
\hline $\mathrm{C}$ & 1.20699722 & 4.39761390 & -0.44701235 \\
\hline $\mathrm{C}$ & -1.20699722 & 4.39761390 & -0.44701235 \\
\hline $\mathrm{C}$ & 0.00000000 & 5.05259123 & -0.22813920 \\
\hline $\mathrm{H}$ & 2.14590405 & 4.91846556 & -0.28295166 \\
\hline $\mathrm{H}$ & -2.14590405 & 4.91846556 & -0.28295166 \\
\hline $\mathrm{H}$ & 0.00000000 & 6.08483863 & 0.10863342 \\
\hline $\mathrm{C}$ & 2.53389831 & 2.36143394 & -1.13202399 \\
\hline $\mathrm{H}$ & 2.64622697 & 1.48951480 & -0.47771398 \\
\hline $\mathrm{H}$ & 2.61257511 & 2.00165740 & -2.16340523 \\
\hline $\mathrm{H}$ & 3.37798285 & 3.02851428 & -0.94732889 \\
\hline $\mathrm{C}$ & 2.53389831 & -2.36143394 & -1.13202399 \\
\hline $\mathrm{H}$ & 2.61257511 & -2.00165740 & -2.16340523 \\
\hline $\mathrm{H}$ & 2.64622697 & -1.48951480 & -0.47771398 \\
\hline $\mathrm{H}$ & 3.37798285 & -3.02851428 & -0.94732889 \\
\hline $\mathrm{C}$ & -2.53389831 & -2.36143394 & -1.13202399 \\
\hline $\mathrm{H}$ & -3.37798285 & -3.02851428 & -0.94732889 \\
\hline $\mathrm{H}$ & -2.64622697 & -1.48951480 & -0.47771398 \\
\hline $\mathrm{H}$ & -2.61257511 & -2.00165740 & -2.16340523 \\
\hline $\mathrm{C}$ & -2.53389831 & 2.36143394 & -1.13202399 \\
\hline $\mathrm{H}$ & -2.61257511 & 2.00165740 & -2.16340523 \\
\hline $\mathrm{H}$ & -2.64622697 & 1.48951480 & -0.47771398 \\
\hline $\mathrm{H}$ & -3.37798285 & 3.02851428 & -0.94732889 \\
\hline $\mathrm{H}$ & -0.92541178 & 1.24751394 & 3.54724191 \\
\hline $\mathrm{H}$ & 0.92541178 & 1.24751394 & 3.5472419 \\
\hline
\end{tabular}


$\left[\left(\mathrm{CH}_{3}-\mathrm{NHC}\right) \mathrm{Au}\left(\mathrm{C}_{2} \mathrm{H}_{4}\right)\right]^{+}$

Cs symmetry; $\mathrm{E}=\mathbf{- 1 0 9 7 . 9 6 3 8 8 5 2}$

\begin{tabular}{|c|c|c|c|}
\hline $\mathrm{Au}$ & -1.42261718 & 0.15680856 & 0.00000000 \\
\hline $\mathrm{C}$ & 0.59665050 & 0.01316296 & 0.00000000 \\
\hline $\mathrm{C}$ & 2.77222306 & 0.53858174 & 0.00000000 \\
\hline $\mathrm{C}$ & 2.69389836 & -0.82229010 & 0.00000000 \\
\hline $\mathrm{C}$ & 1.16493510 & 2.34078158 & -2.53367162 \\
\hline $\mathrm{C}$ & 0.81693206 & -2.36407541 & -2.53356074 \\
\hline $\mathrm{C}$ & 0.81693206 & -2.36407541 & 2.53356074 \\
\hline $\mathrm{C}$ & 1.16493510 & 2.34078158 & 2.53367162 \\
\hline $\mathrm{C}$ & 1.13098264 & 2.42262071 & 0.00000000 \\
\hline $\mathrm{C}$ & 0.97594704 & 3.06893882 & 1.23335744 \\
\hline $\mathrm{C}$ & 0.97594704 & 3.06893882 & -1.23335744 \\
\hline $\mathrm{C}$ & 0.63778452 & 4.42301998 & 1.20677394 \\
\hline $\mathrm{C}$ & 0.63778452 & 4.42301998 & -1.20677394 \\
\hline $\mathrm{C}$ & 0.46898627 & 5.09287080 & 0.00000000 \\
\hline $\mathrm{C}$ & 0.78733097 & -2.44524981 & 0.00000000 \\
\hline $\mathrm{C}$ & 0.53767037 & -3.06246548 & 1.23298589 \\
\hline $\mathrm{C}$ & 0.53767037 & -3.06246548 & -1.23298589 \\
\hline $\mathrm{C}$ & 0.00890588 & -4.35412403 & 1.20678727 \\
\hline $\mathrm{C}$ & 0.00890588 & -4.35412403 & -1.20678727 \\
\hline $\mathrm{C}$ & -0.25292115 & -4.99348575 & 0.00000000 \\
\hline $\mathrm{N}$ & 1.48339864 & 1.02827456 & 0.00000000 \\
\hline $\mathrm{N}$ & 1.33870549 & -1.11749164 & 0.00000000 \\
\hline $\mathrm{H}$ & 3.62933617 & 1.19428032 & 0.00000000 \\
\hline $\mathrm{H}$ & -0.19307013 & -4.86137888 & 2.14579119 \\
\hline $\mathrm{H}$ & -0.19307013 & -4.86137888 & -2.14579119 \\
\hline $\mathrm{H}$ & -0.65942516 & -6.00030024 & 0.00000000 \\
\hline $\mathrm{H}$ & 0.51332314 & 4.95456533 & 2.14579297 \\
\hline $\mathrm{H}$ & 0.51332314 & 4.95456533 & -2.14579297 \\
\hline $\mathrm{H}$ & 0.21098232 & 6.14756154 & 0.00000000 \\
\hline $\mathrm{H}$ & 0.44042623 & 1.52657098 & 2.64824554 \\
\hline $\mathrm{H}$ & 2.16287190 & 1.89618194 & 2.60947427 \\
\hline $\mathrm{H}$ & 1.03864800 & 3.02166114 & 3.37753395 \\
\hline $\mathrm{H}$ & 1.86027170 & -2.03977068 & 2.61075374 \\
\hline $\mathrm{H}$ & 0.19519874 & -1.46895352 & 2.64934674 \\
\hline $\mathrm{H}$ & 0.61097497 & -3.02516711 & 3.37751613 \\
\hline $\mathrm{H}$ & 0.61097497 & -3.02516711 & -3.37751613 \\
\hline $\mathrm{H}$ & 0.19519874 & -1.46895352 & -2.64934674 \\
\hline $\mathrm{H}$ & 1.86027170 & -2.03977068 & -2.61075374 \\
\hline $\mathrm{H}$ & 2.16287190 & 1.89618194 & -2.60947427 \\
\hline $\mathrm{H}$ & 0.44042623 & 1.52657098 & -2.64824554 \\
\hline $\mathrm{H}$ & 1.03864800 & 3.02166114 & -3.37753395 \\
\hline $\mathrm{C}$ & 3.75183775 & -1.86362495 & 0.00000000 \\
\hline $\mathrm{H}$ & 4.73648701 & -1.39341998 & 0.00000000 \\
\hline $\mathrm{H}$ & 3.67890447 & -2.50805847 & 0.88172049 \\
\hline $\mathrm{H}$ & 3.67890447 & -2.50805847 & -0.88172049 \\
\hline $\mathrm{C}$ & -3.55431567 & 0.31158199 & 0.68621277 \\
\hline $\mathrm{C}$ & -3.55431567 & 0.31158199 & -0.68621277 \\
\hline $\mathrm{H}$ & -3.59465854 & 1.24283478 & -1.24667717 \\
\hline $\mathrm{H}$ & -3.72968780 & -0.60392595 & -1.24661473 \\
\hline $\mathrm{H}$ & -3.59465854 & 1.24283478 & 1.24667717 \\
\hline $\mathrm{H}$ & -3.72968780 & -0.60392595 & 1.24661473 \\
\hline
\end{tabular}


$\left[\left(\mathrm{OCH}_{3}-\mathrm{NHC}\right) \mathrm{Au}\left(\mathrm{C}_{2} \mathrm{H}_{4}\right)\right]^{+}$

Cs symmetry; $\mathrm{E}=\mathbf{- 1 1 7 3 . 0 8 5 9 1 4 5}$

\begin{tabular}{|c|c|c|c|}
\hline $\mathrm{Au}$ & -1.55906587 & 0.30059235 & 0.00000000 \\
\hline $\mathrm{C}$ & 0.44583965 & 0.02813399 & 0.00000000 \\
\hline $\mathrm{C}$ & 2.66372522 & 0.40496164 & 0.00000000 \\
\hline $\mathrm{C}$ & 2.46404720 & -0.94797188 & 0.00000000 \\
\hline $\mathrm{C}$ & 1.17037452 & 2.30342534 & -2.53364483 \\
\hline $\mathrm{C}$ & 0.54965787 & -2.39130230 & -2.53019800 \\
\hline $\mathrm{C}$ & 0.54965787 & -2.39130230 & 2.53019800 \\
\hline $\mathrm{C}$ & 1.17037452 & 2.30342534 & 2.53364483 \\
\hline $\mathrm{C}$ & 1.14898678 & 2.38878852 & 0.00000000 \\
\hline $\mathrm{C}$ & 1.04134244 & 3.04456693 & 1.23338582 \\
\hline $\mathrm{C}$ & 1.04134244 & 3.04456693 & -1.23338582 \\
\hline $\mathrm{C}$ & 0.80299387 & 4.41972057 & 1.20682086 \\
\hline $\mathrm{C}$ & 0.80299387 & 4.41972057 & -1.20682086 \\
\hline $\mathrm{C}$ & 0.68361212 & 5.10006927 & 0.00000000 \\
\hline $\mathrm{C}$ & 0.46521073 & -2.45081864 & 0.00000000 \\
\hline $\mathrm{C}$ & 0.17346889 & -3.04720511 & 1.23283269 \\
\hline $\mathrm{C}$ & 0.17346889 & -3.04720511 & -1.23283269 \\
\hline $\mathrm{C}$ & -0.46623164 & -4.28736264 & 1.20699803 \\
\hline $\mathrm{C}$ & -0.46623164 & -4.28736264 & -1.20699803 \\
\hline $\mathrm{C}$ & -0.78623858 & -4.89935504 & 0.00000000 \\
\hline $\mathrm{N}$ & 1.40030195 & 0.97299912 & 0.00000000 \\
\hline $\mathrm{N}$ & 1.10211141 & -1.16127277 & 0.00000000 \\
\hline $\mathrm{H}$ & 3.56136111 & 1.00068277 & 0.00000000 \\
\hline $\mathrm{H}$ & -0.70634185 & -4.77763559 & 2.14602532 \\
\hline $\mathrm{H}$ & -0.70634185 & -4.77763559 & -2.14602532 \\
\hline $\mathrm{H}$ & -1.28100561 & -5.86591520 & 0.00000000 \\
\hline $\mathrm{H}$ & 0.71746926 & 4.95892886 & 2.14583842 \\
\hline $\mathrm{H}$ & 0.71746926 & 4.95892886 & -2.14583842 \\
\hline $\mathrm{H}$ & 0.50368112 & 6.17085581 & 0.00000000 \\
\hline $\mathrm{H}$ & 0.35376244 & 1.58480065 & 2.66776581 \\
\hline $\mathrm{H}$ & 2.10719320 & 1.73955859 & 2.59020398 \\
\hline $\mathrm{H}$ & 1.14554421 & 2.99718140 & 3.37612638 \\
\hline $\mathrm{H}$ & 1.63670145 & -2.28850904 & 2.62085930 \\
\hline $\mathrm{H}$ & 0.11831343 & -1.38837678 & 2.62092039 \\
\hline $\mathrm{H}$ & 0.20114387 & -2.98476122 & 3.37766261 \\
\hline $\mathrm{H}$ & 0.20114387 & -2.98476122 & -3.37766261 \\
\hline $\mathrm{H}$ & 0.11831343 & -1.38837678 & -2.62092039 \\
\hline $\mathrm{H}$ & 1.63670145 & -2.28850904 & -2.62085930 \\
\hline $\mathrm{H}$ & 2.10719320 & 1.73955859 & -2.59020398 \\
\hline $\mathrm{H}$ & 0.35376244 & 1.58480065 & -2.66776581 \\
\hline $\mathrm{H}$ & 1.14554421 & 2.99718140 & -3.37612638 \\
\hline $\mathrm{O}$ & 3.26971908 & -1.99708919 & 0.00000000 \\
\hline $\mathrm{C}$ & 4.66332752 & -1.69586478 & 0.00000000 \\
\hline $\mathrm{H}$ & 5.17818113 & -2.65490190 & 0.00000000 \\
\hline $\mathrm{H}$ & 4.93419180 & -1.12921712 & -0.89742574 \\
\hline $\mathrm{H}$ & 4.93419180 & -1.12921712 & 0.89742574 \\
\hline $\mathrm{C}$ & -3.67536717 & 0.58412266 & 0.68638712 \\
\hline $\mathrm{C}$ & -3.67536717 & 0.58412266 & -0.68638712 \\
\hline $\mathrm{H}$ & -3.66098657 & 1.51618925 & -1.24672032 \\
\hline $\mathrm{H}$ & -3.90528580 & -0.31922508 & -1.24678874 \\
\hline $\mathrm{H}$ & -3.66098657 & 1.51618925 & 1.24672032 \\
\hline $\mathrm{H}$ & -3.90528580 & -0.31922508 & 1.24678874 \\
\hline
\end{tabular}


$\left[\left(\mathrm{NH}_{2}-\mathrm{NHC}\right) \mathrm{Au}\left(\mathrm{C}_{2} \mathbf{H}_{4}\right)\right]^{+}$

C1 symmetry; $\mathrm{E}=\mathbf{- 1 1 1 3 . 9 8 9 1 2 3 6}$

\begin{tabular}{|c|c|c|c|}
\hline $\mathrm{Au}$ & 0.17009516 & 1.42612453 & -0.05576378 \\
\hline $\mathrm{C}$ & 0.01361860 & -0.58735755 & 0.02896966 \\
\hline $\mathrm{C}$ & 0.52929224 & -2.77391138 & 0.10322440 \\
\hline $\mathrm{C}$ & -0.83792270 & -2.67643566 & 0.09791735 \\
\hline $\mathrm{C}$ & 2.31034577 & -1.25187118 & -2.49511426 \\
\hline $\mathrm{C}$ & -2.48833809 & -1.15411544 & -2.45832189 \\
\hline $\mathrm{C}$ & -2.28782179 & -0.52999506 & 2.56394141 \\
\hline $\mathrm{C}$ & 2.34709333 & -1.08564973 & 2.56888704 \\
\hline $\mathrm{C}$ & 2.41173533 & -1.13891884 & 0.03544165 \\
\hline $\mathrm{C}$ & 3.06880987 & -0.95057676 & 1.25837470 \\
\hline $\mathrm{C}$ & 3.05094389 & -1.03254757 & -1.20672330 \\
\hline $\mathrm{C}$ & 4.42606927 & -0.62789555 & 1.21149566 \\
\hline $\mathrm{C}$ & 4.40874078 & -0.70823640 & -1.20058062 \\
\hline $\mathrm{C}$ & 5.08898846 & -0.50635534 & -0.00479842 \\
\hline $\mathrm{C}$ & -2.46078008 & -0.78694302 & 0.04629519 \\
\hline $\mathrm{C}$ & -3.03393522 & -0.39849018 & 1.26626146 \\
\hline $\mathrm{C}$ & -3.13130565 & -0.69412464 & -1.18203306 \\
\hline $\mathrm{C}$ & -4.32921745 & 0.12129778 & 1.22841244 \\
\hline $\mathrm{C}$ & -4.42376015 & -0.16724494 & -1.16724131 \\
\hline $\mathrm{C}$ & -5.01521812 & 0.23861968 & 0.02433409 \\
\hline $\mathrm{N}$ & 1.01493916 & -1.47893535 & 0.05617528 \\
\hline $\mathrm{N}$ & -1.13041517 & -1.32594624 & 0.05543174 \\
\hline $\mathrm{H}$ & 1.18289729 & -3.63065869 & 0.12422268 \\
\hline $\mathrm{H}$ & -4.80219798 & 0.43035910 & 2.15612211 \\
\hline $\mathrm{H}$ & -4.96958402 & -0.08103659 & -2.10230747 \\
\hline $\mathrm{H}$ & -6.02301570 & 0.64277072 & 0.01560471 \\
\hline $\mathrm{H}$ & 4.96565856 & -0.47843437 & 2.14227715 \\
\hline $\mathrm{H}$ & 4.93490021 & -0.62146600 & -2.14687871 \\
\hline $\mathrm{H}$ & 6.14645926 & -0.26040902 & -0.02060475 \\
\hline $\mathrm{H}$ & 1.57085983 & -0.31997632 & 2.67963235 \\
\hline $\mathrm{H}$ & 1.85432047 & -2.05890461 & 2.66255132 \\
\hline $\mathrm{H}$ & 3.04234036 & -0.98000331 & 3.40386927 \\
\hline $\mathrm{H}$ & -1.90247623 & -1.54405594 & 2.71448324 \\
\hline $\mathrm{H}$ & -1.42655106 & 0.14669272 & 2.60092453 \\
\hline $\mathrm{H}$ & -2.93773058 & -0.29011065 & 3.40771157 \\
\hline $\mathrm{H}$ & -3.13068519 & -0.93736149 & -3.31395890 \\
\hline $\mathrm{H}$ & -1.52430099 & -0.66135482 & -2.62438710 \\
\hline $\mathrm{H}$ & -2.30356408 & -2.23400364 & -2.44040993 \\
\hline $\mathrm{H}$ & 1.79640103 & -2.21842416 & -2.50977581 \\
\hline $\mathrm{H}$ & 1.54868093 & -0.48008117 & -2.65504782 \\
\hline $\mathrm{H}$ & 2.99702571 & -1.22356559 & -3.34328803 \\
\hline $\mathrm{N}$ & -1.82633268 & -3.61673649 & 0.04729138 \\
\hline $\mathrm{H}$ & -1.56477496 & -4.55328277 & 0.31144005 \\
\hline $\mathrm{H}$ & -2.73156112 & -3.32890465 & 0.39058290 \\
\hline $\mathrm{C}$ & 0.35619133 & 3.58052625 & 0.53257385 \\
\hline $\mathrm{C}$ & 0.33271547 & 3.51875801 & -0.83915980 \\
\hline $\mathrm{H}$ & 1.25465643 & 3.52597187 & -1.41611911 \\
\hline $\mathrm{H}$ & -0.58991928 & 3.68036554 & -1.39184741 \\
\hline $\mathrm{H}$ & 1.29719684 & 3.63827993 & 1.07484891 \\
\hline $\mathrm{H}$ & -0.54736857 & 3.79351382 & 1.09917636 \\
\hline
\end{tabular}


$\left[(\mathbf{N H C}) \mathbf{A u}\left(\mathrm{NO}_{2}-\mathrm{C}_{2} \mathrm{H}_{3}\right)\right]^{+}$

C1 symmetry; $\mathrm{E}=\mathbf{- 1 2 6 2 . 9 6 2 8 9 3 5}$

\begin{tabular}{|c|c|c|c|}
\hline $\mathrm{Au}$ & -0.04481038 & 0.91462386 & -0.40528842 \\
\hline $\mathrm{C}$ & 0.32350629 & -0.99080932 & 0.15694075 \\
\hline $\mathrm{C}$ & 1.36975445 & -2.86761997 & 0.77905785 \\
\hline $\mathrm{C}$ & 0.03317507 & -3.12146849 & 0.77165378 \\
\hline $\mathrm{C}$ & 2.88203201 & -1.66652052 & -2.13049074 \\
\hline $\mathrm{C}$ & -1.78504001 & -2.62443427 & -2.13704859 \\
\hline $\mathrm{C}$ & -2.07204034 & -0.98718281 & 2.65428003 \\
\hline $\mathrm{C}$ & 2.55336090 & -0.18629751 & 2.70760248 \\
\hline $\mathrm{C}$ & 2.78726508 & -0.86970748 & 0.27557446 \\
\hline $\mathrm{C}$ & 3.29036110 & -0.19933256 & 1.39843864 \\
\hline $\mathrm{C}$ & 3.45046116 & -0.91913348 & -0.95768841 \\
\hline $\mathrm{C}$ & 4.51374311 & 0.45707979 & 1.25212066 \\
\hline $\mathrm{C}$ & 4.67004600 & -0.24664436 & -1.05197194 \\
\hline $\mathrm{C}$ & 5.19537732 & 0.43519362 & 0.04027682 \\
\hline $\mathrm{C}$ & -2.01397733 & -1.78321558 & 0.24528846 \\
\hline $\mathrm{C}$ & -2.73837453 & -1.30921397 & 1.34681147 \\
\hline $\mathrm{C}$ & -2.59939176 & -2.10437884 & -0.98650592 \\
\hline $\mathrm{C}$ & -4.11430106 & -1.14496206 & 1.17942676 \\
\hline $\mathrm{C}$ & -3.97834300 & -1.92330093 & -1.10156809 \\
\hline $\mathrm{C}$ & -4.72729038 & -1.44660616 & -0.03132184 \\
\hline $\mathrm{N}$ & 1.52670766 & -1.55413694 & 0.39840860 \\
\hline $\mathrm{N}$ & -0.59097470 & -1.95679068 & 0.38729159 \\
\hline $\mathrm{H}$ & 2.21259358 & -3.49682125 & 1.02003225 \\
\hline $\mathrm{H}$ & -0.52263718 & -4.01644962 & 1.00493575 \\
\hline $\mathrm{H}$ & -4.70686940 & -0.77833237 & 2.01233373 \\
\hline $\mathrm{H}$ & -4.46559600 & -2.16382666 & -2.04195749 \\
\hline $\mathrm{H}$ & -5.79919373 & -1.31334816 & -0.14031965 \\
\hline $\mathrm{H}$ & 4.93510745 & 0.98350156 & 2.10357497 \\
\hline $\mathrm{H}$ & 5.21292001 & -0.26739278 & -1.99247042 \\
\hline $\mathrm{H}$ & 6.14819951 & 0.94761759 & -0.05152669 \\
\hline $\mathrm{H}$ & 1.57579933 & 0.30108547 & 2.61846574 \\
\hline $\mathrm{H}$ & 2.37452051 & -1.19894914 & 3.08430380 \\
\hline $\mathrm{H}$ & 3.12528866 & 0.35307656 & 3.46481185 \\
\hline $\mathrm{H}$ & -1.49573410 & -1.83485179 & 3.03965551 \\
\hline $\mathrm{H}$ & -1.38019353 & -0.14229455 & 2.55840491 \\
\hline $\mathrm{H}$ & -2.81526724 & -0.71925562 & 3.40733740 \\
\hline $\mathrm{H}$ & -2.41815760 & -2.79183222 & -3.01031647 \\
\hline $\mathrm{H}$ & -0.99548028 & -1.92160567 & -2.42605136 \\
\hline $\mathrm{H}$ & -1.29715721 & -3.57475477 & -1.89460595 \\
\hline $\mathrm{H}$ & 2.76143257 & -2.73320743 & -1.91288985 \\
\hline $\mathrm{H}$ & 1.89612624 & -1.28327531 & -2.41669043 \\
\hline $\mathrm{H}$ & 3.53934466 & -1.57820747 & -2.99744552 \\
\hline $\mathrm{C}$ & -0.44676058 & 3.10169514 & -0.39847539 \\
\hline $\mathrm{C}$ & -0.45419150 & 2.69720385 & -1.70604948 \\
\hline $\mathrm{H}$ & 0.37984051 & 3.58658099 & 0.11165920 \\
\hline $\mathrm{H}$ & 0.44223479 & 2.80123475 & -2.31141700 \\
\hline $\mathrm{H}$ & -1.40003838 & 2.48264681 & -2.19859854 \\
\hline $\mathrm{N}$ & -1.73040483 & 3.28914762 & 0.31991399 \\
\hline $\mathrm{O}$ & -1.64928513 & 3.92587594 & 1.34940956 \\
\hline $\mathrm{O}$ & -2.73078890 & 2.80584546 & -0.17326586 \\
\hline
\end{tabular}


$\left[(\mathrm{NHC}) \mathrm{Au}\left(\mathrm{CF}_{3}-\mathrm{C}_{2} \mathrm{H}_{3}\right)\right]^{+}$

C1 symmetry; $\mathrm{E}=\mathbf{- 1 3 9 5 . 4 0 6 3 1 7 7}$

\begin{tabular}{|c|c|c|c|}
\hline $\mathrm{Au}$ & -0.05547762 & 0.74492407 & -0.52096828 \\
\hline $\mathrm{C}$ & 0.47686047 & -1.07308901 & 0.18909510 \\
\hline $\mathrm{C}$ & 1.68190834 & -2.78928698 & 0.97346651 \\
\hline $\mathrm{C}$ & 0.37619739 & -3.16858813 & 0.97004041 \\
\hline $\mathrm{C}$ & 3.11197680 & -1.64384410 & -2.01855889 \\
\hline $\mathrm{C}$ & -1.41763000 & -3.20012468 & -1.93656050 \\
\hline $\mathrm{C}$ & -1.96766390 & -0.96235049 & 2.58522512 \\
\hline $\mathrm{C}$ & 2.58283735 & 0.10624398 & 2.70912739 \\
\hline $\mathrm{C}$ & 2.91478308 & -0.71070995 & 0.33104056 \\
\hline $\mathrm{C}$ & 3.33811422 & 0.07552191 & 1.41075103 \\
\hline $\mathrm{C}$ & 3.59658647 & -0.77735330 & -0.89104452 \\
\hline $\mathrm{C}$ & 4.49790300 & 0.83156990 & 1.23150230 \\
\hline $\mathrm{C}$ & 4.75062100 & -0.00266837 & -1.01975417 \\
\hline $\mathrm{C}$ & 5.19594796 & 0.79402650 & 0.02939623 \\
\hline $\mathrm{C}$ & -1.77559385 & -2.07975694 & 0.31128328 \\
\hline $\mathrm{C}$ & -2.56594508 & -1.53651119 & 1.33225443 \\
\hline $\mathrm{C}$ & -2.30235287 & -2.61584738 & -0.87205940 \\
\hline $\mathrm{C}$ & -3.94819358 & -1.53390806 & 1.13318761 \\
\hline $\mathrm{C}$ & -3.68908515 & -2.58737096 & -1.02278171 \\
\hline $\mathrm{C}$ & -4.50369285 & -2.05125429 & -0.03099331 \\
\hline $\mathrm{N}$ & 1.72196344 & -1.50037066 & 0.48967781 \\
\hline $\mathrm{N}$ & -0.34661688 & -2.10075575 & 0.48636886 \\
\hline $\mathrm{H}$ & 2.57519785 & -3.31419516 & 1.27473576 \\
\hline $\mathrm{H}$ & -0.09830660 & -4.09042297 & 1.26878378 \\
\hline $\mathrm{H}$ & -4.59101919 & -1.12467894 & 1.90713400 \\
\hline $\mathrm{H}$ & -4.13037825 & -2.99613771 & -1.92711891 \\
\hline $\mathrm{H}$ & -5.58120456 & -2.04317665 & -0.16418285 \\
\hline $\mathrm{H}$ & 4.85674348 & 1.44929681 & 2.04963862 \\
\hline $\mathrm{H}$ & 5.30593248 & -0.03374068 & -1.95268574 \\
\hline $\mathrm{H}$ & 6.09899703 & 1.38525872 & -0.08830634 \\
\hline $\mathrm{H}$ & 1.56044052 & 0.47716833 & 2.57473634 \\
\hline $\mathrm{H}$ & 2.50730165 & -0.88924705 & 3.15951118 \\
\hline $\mathrm{H}$ & 3.08202557 & 0.75819479 & 3.42834642 \\
\hline $\mathrm{H}$ & -1.15600405 & -1.58167978 & 2.97849403 \\
\hline $\mathrm{H}$ & -1.56036936 & 0.03992383 & 2.40680834 \\
\hline $\mathrm{H}$ & -2.72751983 & -0.87065097 & 3.36394842 \\
\hline $\mathrm{H}$ & -2.00777230 & -3.49612626 & -2.80584332 \\
\hline $\mathrm{H}$ & -0.65995742 & -2.48376789 & -2.27282406 \\
\hline $\mathrm{H}$ & -0.88498557 & -4.08889220 & -1.58092651 \\
\hline $\mathrm{H}$ & 3.03973086 & -2.69566953 & -1.72220682 \\
\hline $\mathrm{H}$ & 2.11807061 & -1.33749902 & -2.36418198 \\
\hline $\mathrm{H}$ & 3.79362971 & -1.58458591 & -2.86906278 \\
\hline $\mathrm{C}$ & -0.59741357 & 2.90249732 & -0.62115970 \\
\hline $\mathrm{C}$ & -0.67295367 & 2.40024240 & -1.89697377 \\
\hline $\mathrm{H}$ & 0.16420366 & 2.50937627 & -2.58139249 \\
\hline $\mathrm{H}$ & -1.62486561 & 2.08149251 & -2.31501577 \\
\hline $\mathrm{H}$ & 0.28752459 & 3.43579299 & -0.27811119 \\
\hline $\mathrm{C}$ & -1.81855970 & 3.11097677 & 0.24299448 \\
\hline $\mathrm{F}$ & -2.85922359 & 2.38947994 & -0.18549951 \\
\hline $\mathrm{F}$ & -1.56153738 & 2.75906137 & 1.51121129 \\
\hline $\mathrm{F}$ & -2.15701910 & 4.39838757 & 0.22951244 \\
\hline
\end{tabular}


$\left[(\mathrm{NHC}) \mathrm{Au}\left(\mathrm{Br}-\mathrm{C}_{2} \mathrm{H}_{3}\right)\right]^{+}$

C1 symmetry; $\mathrm{E}=\mathbf{- 3 6 2 9 . 1 5 6 9 8 8 1}$

\begin{tabular}{|c|c|c|c|}
\hline $\mathrm{Au}$ & -0.05504821 & 0.72088069 & -0.52086285 \\
\hline $\mathrm{C}$ & 0.50353645 & -1.08879162 & 0.18384709 \\
\hline $\mathrm{C}$ & 1.74233330 & -2.79657265 & 0.93748187 \\
\hline $\mathrm{C}$ & 0.44651676 & -3.20515430 & 0.91593168 \\
\hline $\mathrm{C}$ & 3.16129512 & -1.55472753 & -2.01932885 \\
\hline $\mathrm{C}$ & -1.32750540 & -3.04388862 & -2.07143620 \\
\hline $\mathrm{C}$ & -1.94860198 & -1.27217246 & 2.63717044 \\
\hline $\mathrm{C}$ & 2.57334675 & 0.07717480 & 2.74250198 \\
\hline $\mathrm{C}$ & 2.93384754 & -0.67962421 & 0.34932715 \\
\hline $\mathrm{C}$ & 3.33711472 & 0.08931213 & 1.44887899 \\
\hline $\mathrm{C}$ & 3.62434150 & -0.70634640 & -0.86935259 \\
\hline $\mathrm{C}$ & 4.48430648 & 0.86958462 & 1.29446466 \\
\hline $\mathrm{C}$ & 4.76519192 & 0.09126086 & -0.97325434 \\
\hline $\mathrm{C}$ & 5.19016578 & 0.87166345 & 0.09634538 \\
\hline $\mathrm{C}$ & -1.72528035 & -2.15172862 & 0.26869676 \\
\hline $\mathrm{C}$ & -2.53434324 & -1.73383444 & 1.33316966 \\
\hline $\mathrm{C}$ & -2.23275808 & -2.59247675 & -0.96072070 \\
\hline $\mathrm{C}$ & -3.91536443 & -1.75794826 & 1.13104832 \\
\hline $\mathrm{C}$ & -3.62013334 & -2.59682466 & -1.11294861 \\
\hline $\mathrm{C}$ & -4.45265488 & -2.18307034 & -0.07877024 \\
\hline $\mathrm{N}$ & 1.75599444 & -1.49553187 & 0.48327224 \\
\hline $\mathrm{N}$ & -0.29756259 & -2.14227475 & 0.45132285 \\
\hline $\mathrm{H}$ & 2.64575912 & -3.30762610 & 1.23213285 \\
\hline $\mathrm{H}$ & -0.00883432 & -4.14507913 & 1.18679220 \\
\hline $\mathrm{H}$ & -4.57200200 & -1.44439607 & 1.93739009 \\
\hline $\mathrm{H}$ & -4.04706247 & -2.93561456 & -2.05244143 \\
\hline $\mathrm{H}$ & -5.52978181 & -2.19893749 & -0.21452593 \\
\hline $\mathrm{H}$ & 4.82715832 & 1.47479819 & 2.12867469 \\
\hline $\mathrm{H}$ & 5.32636999 & 0.09117654 & -1.90320131 \\
\hline $\mathrm{H}$ & 6.08328386 & 1.48120991 & -0.00224015 \\
\hline $\mathrm{H}$ & 1.54483760 & 0.43069235 & 2.60813339 \\
\hline $\mathrm{H}$ & 2.51396852 & -0.92919025 & 3.17056005 \\
\hline $\mathrm{H}$ & 3.05489357 & 0.72302518 & 3.47907321 \\
\hline $\mathrm{H}$ & -1.25515749 & -2.00748584 & 3.05808697 \\
\hline $\mathrm{H}$ & -1.39374202 & -0.33447657 & 2.51713423 \\
\hline $\mathrm{H}$ & -2.73712932 & -1.09859438 & 3.37184030 \\
\hline $\mathrm{H}$ & -1.91089580 & -3.35472250 & -2.94010456 \\
\hline $\mathrm{H}$ & -0.65052498 & -2.24363241 & -2.39119361 \\
\hline $\mathrm{H}$ & -0.70338734 & -3.89171728 & -1.76931040 \\
\hline $\mathrm{H}$ & 3.10505701 & -2.61414223 & -1.74752851 \\
\hline $\mathrm{H}$ & 2.16421348 & -1.25683398 & -2.36306912 \\
\hline $\mathrm{H}$ & 3.84666724 & -1.46438513 & -2.86411492 \\
\hline $\mathrm{C}$ & -0.62998423 & 2.90359626 & -0.60879011 \\
\hline $\mathrm{C}$ & -0.68266123 & 2.38331969 & -1.87754445 \\
\hline $\mathrm{H}$ & 0.17166188 & 2.51653277 & -2.53808718 \\
\hline $\mathrm{H}$ & -1.62331588 & 2.07023442 & -2.32287932 \\
\hline $\mathrm{H}$ & 0.24636872 & 3.42743511 & -0.23588544 \\
\hline $\mathrm{Br}$ & -2.14613929 & 3.14257988 & 0.47601303 \\
\hline
\end{tabular}


$\left[(\mathrm{NHC}) \mathrm{Au}\left(\mathrm{CH}_{3}-\mathrm{C}_{2} \mathrm{H}_{3}\right)\right]^{+}$

C1 symmetry; $\mathrm{E}=\mathbf{- 1 0 9 7 . 9 6 1 7 6 8 4}$

\begin{tabular}{|c|c|c|c|}
\hline $\mathrm{Au}$ & -0.01568763 & 1.16426495 & -0.21622129 \\
\hline $\mathrm{C}$ & 0.09692161 & -0.83710689 & 0.06306912 \\
\hline $\mathrm{C}$ & 0.89558627 & -2.91321348 & 0.34699440 \\
\hline $\mathrm{C}$ & -0.46026413 & -2.99303720 & 0.32937714 \\
\hline $\mathrm{C}$ & 2.52329626 & -1.41898604 & -2.36963244 \\
\hline $\mathrm{C}$ & -2.17402278 & -1.64396287 & -2.43479502 \\
\hline $\mathrm{C}$ & -2.30334518 & -1.11532881 & 2.60312653 \\
\hline $\mathrm{C}$ & 2.43321129 & -0.78394653 & 2.65698388 \\
\hline $\mathrm{C}$ & 2.55649513 & -1.05872451 & 0.13982551 \\
\hline $\mathrm{C}$ & 3.15540308 & -0.66901825 & 1.34479322 \\
\hline $\mathrm{C}$ & 3.19932768 & -0.97894062 & -1.10234397 \\
\hline $\mathrm{C}$ & 4.45657944 & -0.16815701 & 1.27789583 \\
\hline $\mathrm{C}$ & 4.49968377 & -0.47155695 & -1.11626676 \\
\hline $\mathrm{C}$ & 5.12139123 & -0.06917973 & 0.06059464 \\
\hline $\mathrm{C}$ & -2.32090710 & -1.34514738 & 0.07864075 \\
\hline $\mathrm{C}$ & -2.99595168 & -1.05481584 & 1.27144680 \\
\hline $\mathrm{C}$ & -2.93294818 & -1.31398424 & -1.18111231 \\
\hline $\mathrm{C}$ & -4.34455972 & -0.70833554 & 1.17367433 \\
\hline $\mathrm{C}$ & -4.28308931 & -0.96196084 & -1.22594213 \\
\hline $\mathrm{C}$ & -4.98132214 & -0.66102758 & -0.06171239 \\
\hline $\mathrm{N}$ & 1.21704599 & -1.58276914 & 0.18204039 \\
\hline $\mathrm{N}$ & -0.93100550 & -1.70915687 & 0.15358283 \\
\hline $\mathrm{H}$ & 1.65277085 & -3.67348613 & 0.46007287 \\
\hline $\mathrm{H}$ & -1.12570583 & -3.83706420 & 0.42384445 \\
\hline $\mathrm{H}$ & -4.89846365 & -0.48143532 & 2.08006029 \\
\hline $\mathrm{H}$ & -4.78913984 & -0.93243922 & -2.18656597 \\
\hline $\mathrm{H}$ & -6.03264482 & -0.39521579 & -0.11680756 \\
\hline $\mathrm{H}$ & 4.95136308 & 0.14005450 & 2.19438370 \\
\hline $\mathrm{H}$ & 5.02793665 & -0.39937253 & -2.06255877 \\
\hline $\mathrm{H}$ & 6.13520239 & 0.31831953 & 0.02973115 \\
\hline $\mathrm{H}$ & 1.52284898 & -0.17405944 & 2.67026179 \\
\hline $\mathrm{H}$ & 2.13221607 & -1.81583034 & 2.86710338 \\
\hline $\mathrm{H}$ & 3.07136152 & -0.45053213 & 3.47748960 \\
\hline $\mathrm{H}$ & -1.91484854 & -2.11739503 & 2.81430206 \\
\hline $\mathrm{H}$ & -1.45308490 & -0.42554495 & 2.64748524 \\
\hline $\mathrm{H}$ & -2.99251629 & -0.85110988 & 3.40745212 \\
\hline $\mathrm{H}$ & -2.83828548 & -1.63107573 & -3.30094183 \\
\hline $\mathrm{H}$ & -1.36955614 & -0.92279876 & -2.61924105 \\
\hline $\mathrm{H}$ & -1.71189339 & -2.63534212 & -2.38256581 \\
\hline $\mathrm{H}$ & 2.20883481 & -2.46691501 & -2.32106778 \\
\hline $\mathrm{H}$ & 1.62708586 & -0.82283361 & -2.57568042 \\
\hline $\mathrm{H}$ & 3.19760087 & -1.31331221 & -3.22153579 \\
\hline $\mathrm{C}$ & -0.22939571 & 3.40576416 & 0.24744658 \\
\hline $\mathrm{C}$ & -0.07893244 & 3.20418162 & -1.10571606 \\
\hline $\mathrm{H}$ & 0.89852832 & 3.29357914 & -1.57452633 \\
\hline $\mathrm{H}$ & -0.94400407 & 3.21371775 & -1.76672087 \\
\hline $\mathrm{H}$ & 0.66984800 & 3.59224741 & 0.83688826 \\
\hline $\mathrm{C}$ & -1.53330270 & 3.65861955 & 0.93476920 \\
\hline $\mathrm{H}$ & -1.59676114 & 4.72790320 & 1.17236605 \\
\hline $\mathrm{H}$ & -2.38872644 & 3.39443137 & 0.30893612 \\
\hline $\mathrm{H}$ & -1.60152532 & 3.11927706 & 1.88381015 \\
\hline
\end{tabular}


$\left[(\mathrm{NHC}) \mathrm{Au}\left(\mathrm{OCH}_{3}-\mathrm{C}_{2} \mathrm{H}_{3}\right)\right]^{+}$

C1 symmetry; $\mathrm{E}=\mathbf{- 1 1 7 3 . 0 9 6 3 9 5 6}$

\begin{tabular}{|c|c|c|c|}
\hline $\mathrm{Au}$ & -0.05436224 & 1.04691831 & -0.24815067 \\
\hline $\mathrm{C}$ & 0.33492628 & -0.91078417 & 0.07204967 \\
\hline $\mathrm{C}$ & 1.39180353 & -2.86584824 & 0.38215159 \\
\hline $\mathrm{C}$ & 0.05824222 & -3.11857381 & 0.37186066 \\
\hline $\mathrm{C}$ & 2.80111525 & -1.18678163 & -2.36426972 \\
\hline $\mathrm{C}$ & -1.82709573 & -2.08787673 & -2.39601028 \\
\hline $\mathrm{C}$ & -1.99189798 & -1.38355099 & 2.61755559 \\
\hline $\mathrm{C}$ & 2.65250845 & -0.54125539 & 2.65829274 \\
\hline $\mathrm{C}$ & 2.80160602 & -0.81619633 & 0.14334001 \\
\hline $\mathrm{C}$ & 3.35112910 & -0.34576557 & 1.34296894 \\
\hline $\mathrm{C}$ & 3.42336168 & -0.66084916 & -1.10225332 \\
\hline $\mathrm{C}$ & 4.57773526 & 0.31597641 & 1.26741520 \\
\hline $\mathrm{C}$ & 4.64858756 & 0.00759581 & -1.12535752 \\
\hline $\mathrm{C}$ & 5.21948910 & 0.49175144 & 0.04639298 \\
\hline $\mathrm{C}$ & -1.99590034 & -1.71623034 & 0.10529488 \\
\hline $\mathrm{C}$ & -2.69482271 & -1.46098714 & 1.29248691 \\
\hline $\mathrm{C}$ & -2.61431266 & -1.80256826 & -1.14901068 \\
\hline $\mathrm{C}$ & -4.07549821 & -1.27755342 & 1.19478051 \\
\hline $\mathrm{C}$ & -3.99663794 & -1.61110714 & -1.19486807 \\
\hline $\mathrm{C}$ & -4.71984125 & -1.35092642 & -0.03559861 \\
\hline $\mathrm{N}$ & 1.54048039 & -1.50628176 & 0.19597057 \\
\hline $\mathrm{N}$ & -0.57268616 & -1.90617713 & 0.17907059 \\
\hline $\mathrm{H}$ & 2.24043401 & -3.52095982 & 0.50328293 \\
\hline $\mathrm{H}$ & -0.49380916 & -4.03883979 & 0.48309540 \\
\hline $\mathrm{H}$ & -4.64743907 & -1.08519674 & 2.09805079 \\
\hline $\mathrm{H}$ & -4.50740624 & -1.67832027 & -2.15120068 \\
\hline $\mathrm{H}$ & -5.79590500 & -1.21512690 & -0.09024281 \\
\hline $\mathrm{H}$ & 5.03279489 & 0.69029848 & 2.17988132 \\
\hline $\mathrm{H}$ & 5.15884732 & 0.14173094 & -2.07478177 \\
\hline $\mathrm{H}$ & 6.17569927 & 1.00477096 & 0.00854011 \\
\hline $\mathrm{H}$ & 1.67088200 & -0.05425059 & 2.66750275 \\
\hline $\mathrm{H}$ & 2.48777735 & -1.60142769 & 2.87845248 \\
\hline $\mathrm{H}$ & 3.24343491 & -0.12041035 & 3.47400745 \\
\hline $\mathrm{H}$ & -1.44043982 & -2.30309456 & 2.83986652 \\
\hline $\mathrm{H}$ & -1.26831380 & -0.56086815 & 2.63913215 \\
\hline $\mathrm{H}$ & -2.70848642 & -1.22123103 & 3.42492400 \\
\hline $\mathrm{H}$ & -2.48798042 & -2.15247645 & -3.26248702 \\
\hline $\mathrm{H}$ & -1.08896165 & -1.30224153 & -2.59324191 \\
\hline $\mathrm{H}$ & -1.27862175 & -3.03285271 & -2.32305803 \\
\hline $\mathrm{H}$ & 2.59963166 & -2.26142118 & -2.30277834 \\
\hline $\mathrm{H}$ & 1.84707663 & -0.69112991 & -2.57723912 \\
\hline $\mathrm{H}$ & 3.46154774 & -1.01958256 & -3.21722726 \\
\hline $\mathrm{C}$ & -0.87549954 & 3.32216090 & 0.40724210 \\
\hline $\mathrm{C}$ & -0.44059561 & 3.09972849 & -0.89115617 \\
\hline $\mathrm{H}$ & 0.55743641 & 3.45993604 & -1.13126339 \\
\hline $\mathrm{H}$ & -1.14623627 & 3.05021966 & -1.71618800 \\
\hline $\mathrm{H}$ & -0.17283744 & 3.61243792 & 1.18653507 \\
\hline $\mathrm{O}$ & -2.10100179 & 3.28520356 & 0.87137119 \\
\hline $\mathrm{C}$ & -3.16277574 & 2.93929497 & -0.02035999 \\
\hline $\mathrm{H}$ & -3.01231110 & 1.92236726 & -0.40094798 \\
\hline $\mathrm{H}$ & -4.07317978 & 2.98482091 & 0.57376823 \\
\hline $\mathrm{H}$ & -3.21932266 & 3.65396581 & -0.846422 \\
\hline
\end{tabular}


$\left[(\mathrm{NHC}) \mathrm{Au}\left(\mathrm{NH}_{2}-\mathrm{C}_{2} \mathbf{H}_{3}\right)\right]^{+}$

C1 symmetry; $\mathrm{E}=\mathbf{- 1 1 1 4 . 0 0 5 7 6 4 8}$

\begin{tabular}{|c|c|c|c|}
\hline $\mathrm{Au}$ & -0.02155374 & 1.15864564 & -0.18432067 \\
\hline $\mathrm{C}$ & 0.16069278 & -0.84465105 & 0.04700732 \\
\hline $\mathrm{C}$ & 1.04640571 & -2.90044135 & 0.25407478 \\
\hline $\mathrm{C}$ & -0.30292370 & -3.03676290 & 0.23688495 \\
\hline $\mathrm{C}$ & 2.59224855 & -1.22437180 & -2.41125242 \\
\hline $\mathrm{C}$ & -2.07853814 & -1.62874295 & -2.47166319 \\
\hline $\mathrm{C}$ & -2.21489252 & -1.35151151 & 2.58193063 \\
\hline $\mathrm{C}$ & 2.50242159 & -0.79781967 & 2.63417848 \\
\hline $\mathrm{C}$ & 2.62716517 & -0.97337148 & 0.10999602 \\
\hline $\mathrm{C}$ & 3.21799087 & -0.61015867 & 1.32696615 \\
\hline $\mathrm{C}$ & 3.26125519 & -0.81816988 & -1.12926974 \\
\hline $\mathrm{C}$ & 4.50015284 & -0.06102128 & 1.27717988 \\
\hline $\mathrm{C}$ & 4.54267481 & -0.26459357 & -1.12712725 \\
\hline $\mathrm{C}$ & 5.15571927 & 0.11086048 & 0.06298870 \\
\hline $\mathrm{C}$ & -2.23150277 & -1.46397964 & 0.05171263 \\
\hline $\mathrm{C}$ & -2.91746584 & -1.26643216 & 1.25704678 \\
\hline $\mathrm{C}$ & -2.85056562 & -1.40297129 & -1.20332535 \\
\hline $\mathrm{C}$ & -4.28324603 & -0.98851046 & 1.17913190 \\
\hline $\mathrm{C}$ & -4.21790910 & -1.12171432 & -1.22977644 \\
\hline $\mathrm{C}$ & -4.92729195 & -0.91667494 & -0.05145005 \\
\hline $\mathrm{N}$ & 1.31019713 & -1.55010985 & 0.13618311 \\
\hline $\mathrm{N}$ & -0.82774243 & -1.76538789 & 0.10822237 \\
\hline $\mathrm{H}$ & 1.83604841 & -3.63080743 & 0.33744119 \\
\hline $\mathrm{H}$ & -0.93247292 & -3.91044770 & 0.30212194 \\
\hline $\mathrm{H}$ & -4.84491948 & -0.83857696 & 2.09689140 \\
\hline $\mathrm{H}$ & -4.72860520 & -1.07494873 & -2.18740730 \\
\hline $\mathrm{H}$ & -5.99260187 & -0.70968656 & -0.09202081 \\
\hline $\mathrm{H}$ & 4.98725739 & 0.22806826 & 2.20403462 \\
\hline $\mathrm{H}$ & 5.06271129 & -0.13380641 & -2.07172844 \\
\hline $\mathrm{H}$ & 6.15502331 & 0.53521727 & 0.04466140 \\
\hline $\mathrm{H}$ & 1.57731648 & -0.21142146 & 2.67012589 \\
\hline $\mathrm{H}$ & 2.22680333 & -1.84447059 & 2.80160585 \\
\hline $\mathrm{H}$ & 3.13350120 & -0.48211049 & 3.46721963 \\
\hline $\mathrm{H}$ & -1.78184373 & -2.34358045 & 2.74881850 \\
\hline $\mathrm{H}$ & -1.39394134 & -0.62888587 & 2.64731604 \\
\hline $\mathrm{H}$ & -2.90924296 & -1.15123502 & 3.40037538 \\
\hline $\mathrm{H}$ & -2.74495578 & -1.61498771 & -3.33629939 \\
\hline $\mathrm{H}$ & -1.31837972 & -0.85319644 & -2.61926974 \\
\hline $\mathrm{H}$ & -1.55789183 & -2.59198250 & -2.46469508 \\
\hline $\mathrm{H}$ & 2.29319550 & -2.27779103 & -2.39939452 \\
\hline $\mathrm{H}$ & 1.68716275 & -0.63388631 & -2.59351244 \\
\hline $\mathrm{H}$ & 3.26404129 & -1.07745032 & -3.25912467 \\
\hline $\mathrm{C}$ & -0.90689644 & 3.60624452 & 0.48324077 \\
\hline $\mathrm{C}$ & -0.11756007 & 3.26772057 & -0.62443326 \\
\hline $\mathrm{H}$ & 0.92947217 & 3.55997444 & -0.55084628 \\
\hline $\mathrm{H}$ & -0.55758015 & 3.38802913 & -1.61490729 \\
\hline $\mathrm{H}$ & -0.42951973 & 3.73393275 & 1.45340782 \\
\hline $\mathrm{N}$ & -2.21521056 & 3.81001354 & 0.48155414 \\
\hline $\mathrm{H}$ & -2.72182902 & 3.96241387 & 1.33850922 \\
\hline $\mathrm{H}$ & -2.75432030 & 3.72946035 & -0.36795540 \\
\hline
\end{tabular}


$\left[(\mathbf{N H C}) \mathbf{A u}\left(\mathrm{NO}_{2}-\mathrm{C}_{8} \mathrm{H}_{7}\right)\right]^{+}$

C1 symmetry; $\mathrm{E}=\mathbf{- 1 4 9 3 . 7 6 8 9 9 6 8}$

\begin{tabular}{|c|c|c|c|}
\hline $\mathrm{Au}$ & -0.56029853 & -0.52022308 & -0.79464664 \\
\hline $\mathrm{C}$ & -1.49368513 & 0.83974281 & 0.37324260 \\
\hline $\mathrm{C}$ & -1.91638082 & 2.69753578 & 1.55486601 \\
\hline $\mathrm{C}$ & -3.00797308 & 1.89379105 & 1.64565348 \\
\hline $\mathrm{C}$ & 1.22142799 & 1.36199372 & 2.46681983 \\
\hline $\mathrm{C}$ & -2.64655165 & -1.34240290 & 2.86624492 \\
\hline $\mathrm{C}$ & -4.50586419 & 0.78126515 & -1.34254678 \\
\hline $\mathrm{C}$ & -0.77718655 & 3.66137373 & -1.58344529 \\
\hline $\mathrm{C}$ & 0.30258024 & 2.53550056 & 0.41553290 \\
\hline $\mathrm{C}$ & 0.41601371 & 3.33077865 & -0.73272474 \\
\hline $\mathrm{C}$ & 1.38748246 & 2.21575994 & 1.24222814 \\
\hline $\mathrm{C}$ & 1.68722119 & 3.80985854 & -1.05430178 \\
\hline $\mathrm{C}$ & 2.63780758 & 2.71974524 & 0.87851276 \\
\hline $\mathrm{C}$ & 2.78655805 & 3.50792388 & -0.25745384 \\
\hline $\mathrm{C}$ & -3.62391427 & -0.35446355 & 0.74578574 \\
\hline $\mathrm{C}$ & -4.49506386 & -0.34733484 & -0.35122991 \\
\hline $\mathrm{C}$ & -3.58833928 & -1.38277244 & 1.69650892 \\
\hline $\mathrm{C}$ & -5.35631031 & -1.43725740 & -0.48864258 \\
\hline $\mathrm{C}$ & -4.46898178 & -2.45032331 & 1.51450887 \\
\hline $\mathrm{C}$ & -5.34287450 & -2.47813549 & 0.43318723 \\
\hline $\mathrm{N}$ & -0.99872737 & 2.03297537 & 0.76964451 \\
\hline $\mathrm{N}$ & -2.72877786 & 0.75978960 & 0.91314228 \\
\hline $\mathrm{H}$ & -1.71121133 & 3.67045121 & 1.97382331 \\
\hline $\mathrm{H}$ & -3.94805689 & 2.02441437 & 2.15882005 \\
\hline $\mathrm{H}$ & -6.04762244 & -1.46135824 & -1.32603514 \\
\hline $\mathrm{H}$ & -4.46974805 & -3.26281231 & 2.23535107 \\
\hline $\mathrm{H}$ & -6.02415417 & -3.31481717 & 0.31181052 \\
\hline $\mathrm{H}$ & 1.80858587 & 4.43471021 & -1.93448652 \\
\hline $\mathrm{H}$ & 3.49961748 & 2.49522167 & 1.50054381 \\
\hline $\mathrm{H}$ & 3.76566084 & 3.89886314 & -0.51799821 \\
\hline $\mathrm{H}$ & -1.25520229 & 2.75766638 & -1.97772922 \\
\hline $\mathrm{H}$ & -1.53993100 & 4.20806224 & -1.01865712 \\
\hline $\mathrm{H}$ & -0.48437728 & 4.28299798 & -2.43164987 \\
\hline $\mathrm{H}$ & -4.67316057 & 1.74879059 & -0.85770382 \\
\hline $\mathrm{H}$ & -3.55425015 & 0.85058652 & -1.88200339 \\
\hline $\mathrm{H}$ & -5.29730091 & 0.63706031 & -2.08039246 \\
\hline $\mathrm{H}$ & -2.76060098 & -2.23626924 & 3.48239508 \\
\hline $\mathrm{H}$ & -1.60139304 & -1.28819521 & 2.54164658 \\
\hline $\mathrm{H}$ & -2.82987977 & -0.47258048 & 3.50623135 \\
\hline $\mathrm{H}$ & 0.44814609 & 1.75284138 & 3.13613265 \\
\hline $\mathrm{H}$ & 0.93377263 & 0.33757436 & 2.20338275 \\
\hline $\mathrm{H}$ & 2.15537435 & 1.31174591 & 3.02976267 \\
\hline $\mathrm{C}$ & 0.23159000 & -1.66617184 & -2.53259633 \\
\hline $\mathrm{C}$ & 0.84091679 & -2.22790111 & -1.42793751 \\
\hline $\mathrm{H}$ & 0.36794423 & -3.10755148 & -0.99032180 \\
\hline $\mathrm{H}$ & 0.73937240 & -0.93739137 & -3.15952787 \\
\hline $\mathrm{H}$ & -0.64477333 & -2.14410838 & -2.96214663 \\
\hline $\mathrm{C}$ & 2.16960296 & -1.90515633 & -0.88266856 \\
\hline $\mathrm{C}$ & 2.70319845 & -2.75247663 & 0.09884297 \\
\hline $\mathrm{C}$ & 2.91034565 & -0.78875384 & -1.30343725 \\
\hline $\mathrm{C}$ & 3.95082235 & -2.49979203 & 0.65349694 \\
\hline $\mathrm{C}$ & 4.64839843 & -1.38093140 & 0.22245232 \\
\hline $\mathrm{C}$ & 4.15085125 & -0.52050797 & -0.75044655 \\
\hline $\mathrm{H}$ & 2.13904187 & -3.62187090 & 0.42512350 \\
\hline $\mathrm{H}$ & 2.51229250 & -0.11264893 & -2.05371816 \\
\hline $\mathrm{H}$ & 4.38673144 & -3.14310096 & 1.40893633 \\
\hline $\mathrm{H}$ & 4.73753635 & 0.34000108 & -1.04895169 \\
\hline $\mathrm{N}$ & 5.95362135 & -1.08195415 & 0.82739990 \\
\hline $\mathrm{O}$ & 6.50305270 & -0.05261988 & 0.47615166 \\
\hline $\mathrm{O}$ & 6.38422320 & -1.87883797 & 1.64015146 \\
\hline
\end{tabular}


$\left[(\mathrm{NHC}) \mathrm{Au}\left(\mathrm{CF}_{3}-\mathrm{C}_{8} \mathrm{H}_{7}\right)\right]^{+}$

C1 symmetry; $\mathrm{E}=\mathbf{- 1 6 2 6 . 2 0 3 5 4 3 6}$

\begin{tabular}{|c|c|c|c|}
\hline $\mathrm{Au}$ & -0.80759046 & -0.46243343 & -0.94513337 \\
\hline $\mathrm{C}$ & -1.55985845 & 0.82246043 & 0.42082282 \\
\hline $\mathrm{C}$ & -1.74644911 & 2.54730701 & 1.84149122 \\
\hline $\mathrm{C}$ & -2.86149211 & 1.78301866 & 1.97323586 \\
\hline $\mathrm{C}$ & 1.45484263 & 0.94716854 & 2.12041260 \\
\hline $\mathrm{C}$ & -2.56034426 & -1.55465536 & 2.82034435 \\
\hline $\mathrm{C}$ & -4.71672635 & 1.04792785 & -0.95460975 \\
\hline $\mathrm{C}$ & -0.98440614 & 3.89808786 & -1.20840949 \\
\hline $\mathrm{C}$ & 0.30636335 & 2.44684166 & 0.42246063 \\
\hline $\mathrm{C}$ & 0.29774428 & 3.40849062 & -0.59736065 \\
\hline $\mathrm{C}$ & 1.48032390 & 1.96869801 & 1.01925731 \\
\hline $\mathrm{C}$ & 1.53274736 & 3.89846180 & -1.02479307 \\
\hline $\mathrm{C}$ & 2.69105556 & 2.48623591 & 0.55337498 \\
\hline $\mathrm{C}$ & 2.71682633 & 3.44243759 & -0.45549940 \\
\hline $\mathrm{C}$ & -3.69248322 & -0.32226173 & 0.91645783 \\
\hline $\mathrm{C}$ & -4.66789523 & -0.17040154 & -0.07733664 \\
\hline $\mathrm{C}$ & -3.61663908 & -1.43939520 & 1.75837661 \\
\hline $\mathrm{C}$ & -5.59576492 & -1.20306398 & -0.22353770 \\
\hline $\mathrm{C}$ & -4.56681514 & -2.44469940 & 1.57234232 \\
\hline $\mathrm{C}$ & -5.54549880 & -2.32853900 & 0.59129550 \\
\hline $\mathrm{N}$ & -0.96021587 & 1.94173862 & 0.88457237 \\
\hline $\mathrm{N}$ & -2.72770668 & 0.73037982 & 1.09269261 \\
\hline $\mathrm{H}$ & -1.44395842 & 3.45712624 & 2.33623798 \\
\hline $\mathrm{H}$ & -3.72921120 & 1.89138715 & 2.60519545 \\
\hline $\mathrm{H}$ & -6.36799847 & -1.11509292 & -0.98224896 \\
\hline $\mathrm{H}$ & -4.53810672 & -3.32312579 & 2.21057932 \\
\hline $\mathrm{H}$ & -6.27932148 & -3.11882110 & 0.46526134 \\
\hline $\mathrm{H}$ & 1.55958267 & 4.64914899 & -1.80939533 \\
\hline $\mathrm{H}$ & 3.61777691 & 2.12658951 & 0.99066330 \\
\hline $\mathrm{H}$ & 3.66755116 & 3.84051356 & -0.79770319 \\
\hline $\mathrm{H}$ & -1.57012437 & 3.07467680 & -1.63176268 \\
\hline $\mathrm{H}$ & -1.62099450 & 4.40045933 & -0.47202472 \\
\hline $\mathrm{H}$ & -0.78035330 & 4.61090905 & -2.00958099 \\
\hline $\mathrm{H}$ & -4.75216935 & 1.97205592 & -0.36834112 \\
\hline $\mathrm{H}$ & -3.83492358 & 1.10959574 & -1.60270079 \\
\hline $\mathrm{H}$ & -5.59999749 & 1.02479237 & -1.59566371 \\
\hline $\mathrm{H}$ & -2.64789570 & -2.50645915 & 3.34777428 \\
\hline $\mathrm{H}$ & -1.55236222 & -1.49558255 & 2.39477750 \\
\hline $\mathrm{H}$ & -2.64197795 & -0.75459766 & 3.56403641 \\
\hline $\mathrm{H}$ & 0.94765510 & 1.32858978 & 3.01357418 \\
\hline $\mathrm{H}$ & 0.93119614 & 0.03549067 & 1.81332583 \\
\hline $\mathrm{H}$ & 2.47170816 & 0.66897169 & 2.40389160 \\
\hline $\mathrm{C}$ & -0.13251080 & -1.52645048 & -2.79000220 \\
\hline $\mathrm{C}$ & 0.48764496 & -2.18114022 & -1.74378703 \\
\hline $\mathrm{H}$ & -0.00729265 & -3.06792713 & -1.34617626 \\
\hline $\mathrm{H}$ & 0.37546671 & -0.76230324 & -3.37331436 \\
\hline $\mathrm{H}$ & -1.02978604 & -1.95198385 & -3.23056168 \\
\hline $\mathrm{C}$ & 1.81752820 & -1.90837088 & -1.17718472 \\
\hline $\mathrm{C}$ & 2.21061302 & -2.62574698 & -0.03887724 \\
\hline $\mathrm{C}$ & 2.67874626 & -0.93177321 & -1.69903451 \\
\hline $\mathrm{C}$ & 3.40968362 & -2.33879083 & 0.59972384 \\
\hline $\mathrm{C}$ & 4.22980198 & -1.33226625 & 0.09766809 \\
\hline $\mathrm{C}$ & 3.87613091 & -0.64547812 & -1.06482135 \\
\hline $\mathrm{H}$ & 1.56349823 & -3.40495028 & 0.35544356 \\
\hline $\mathrm{H}$ & 2.41203451 & -0.38444654 & -2.59759202 \\
\hline $\mathrm{H}$ & 3.70743480 & -2.88923399 & 1.48579967 \\
\hline $\mathrm{H}$ & 4.53868154 & 0.11437775 & -1.46514372 \\
\hline $\mathrm{C}$ & 5.45945902 & -0.91694794 & 0.85734556 \\
\hline $\mathrm{F}$ & 5.15689707 & 0.08715600 & 1.71079962 \\
\hline $\mathrm{F}$ & 6.41803858 & -0.46585838 & 0.04339067 \\
\hline $\mathrm{F}$ & 5.95880597 & -1.91754847 & 1.58633216 \\
\hline
\end{tabular}


$\left[(\mathrm{NHC}) \mathrm{Au}\left(\mathrm{Br}-\mathrm{C}_{8} \mathrm{H}_{7}\right)\right]^{+}$

C1 symmetry; $\mathrm{E}=\mathbf{- 3 8 5 9 . 9 5 6 7 5 0 2}$

\begin{tabular}{|c|c|c|c|}
\hline $\mathrm{Au}$ & -0.79501261 & -0.42701487 & -0.86656247 \\
\hline $\mathrm{C}$ & -1.78375798 & 0.78322603 & 0.41543860 \\
\hline $\mathrm{C}$ & -2.32154961 & 2.53343711 & 1.71154448 \\
\hline $\mathrm{C}$ & -3.32359091 & 1.62245356 & 1.81368885 \\
\hline $\mathrm{C}$ & -3.76549959 & -0.63169023 & 0.83181399 \\
\hline $\mathrm{C}$ & -4.68922184 & -0.66443550 & -0.22067574 \\
\hline $\mathrm{C}$ & -3.58086601 & -1.69150322 & 1.72911437 \\
\hline $\mathrm{C}$ & -5.44694980 & -1.82745526 & -0.36860511 \\
\hline $\mathrm{C}$ & -4.36208701 & -2.83241543 & 1.53897218 \\
\hline $\mathrm{C}$ & -5.28463972 & -2.90041871 & 0.50070201 \\
\hline $\mathrm{C}$ & -0.16257597 & 2.64705022 & 0.45933300 \\
\hline $\mathrm{C}$ & -0.19698512 & 3.52782954 & -0.62999600 \\
\hline $\mathrm{C}$ & 0.99839782 & 2.37572055 & 1.19456694 \\
\hline $\mathrm{C}$ & 1.00044413 & 4.15030858 & -0.98613817 \\
\hline $\mathrm{C}$ & 2.17042782 & 3.02325574 & 0.79910891 \\
\hline $\mathrm{C}$ & 2.17188810 & 3.90103182 & -0.27910893 \\
\hline $\mathrm{C}$ & -1.46679682 & 3.79478654 & -1.38682013 \\
\hline $\mathrm{C}$ & -4.85918083 & 0.49805747 & -1.15668583 \\
\hline $\mathrm{C}$ & -2.58640669 & -1.60799343 & 2.85200121 \\
\hline $\mathrm{C}$ & 0.99390914 & 1.42154604 & 2.35449969 \\
\hline $\mathrm{N}$ & -1.38689165 & 1.99983529 & 0.84926268 \\
\hline $\mathrm{N}$ & -2.97527258 & 0.55712487 & 1.01024172 \\
\hline $\mathrm{H}$ & -2.18860840 & 3.50106557 & 2.16988500 \\
\hline $\mathrm{H}$ & -4.24298070 & 1.63468266 & 2.37817221 \\
\hline $\mathrm{H}$ & -6.17514464 & -1.88418450 & -1.17251160 \\
\hline $\mathrm{H}$ & -4.24603900 & -3.67124181 & 2.21920765 \\
\hline $\mathrm{H}$ & -5.88699127 & -3.79461048 & 0.37207194 \\
\hline $\mathrm{H}$ & 1.00675392 & 4.84292943 & -1.82284711 \\
\hline $\mathrm{H}$ & 3.08692598 & 2.83835266 & 1.35208905 \\
\hline $\mathrm{H}$ & 3.09155331 & 4.40233124 & -0.56580172 \\
\hline $\mathrm{H}$ & -1.88344175 & 2.87445084 & -1.81096797 \\
\hline $\mathrm{H}$ & -2.23926842 & 4.23182044 & -0.74498863 \\
\hline $\mathrm{H}$ & -1.28587853 & 4.49075857 & -2.20812135 \\
\hline $\mathrm{H}$ & -5.08119247 & 1.42591928 & -0.61922132 \\
\hline $\mathrm{H}$ & -3.95102190 & 0.67448696 & -1.74449903 \\
\hline $\mathrm{H}$ & -5.67750238 & 0.31283338 & -1.85504487 \\
\hline $\mathrm{H}$ & -2.57723962 & -2.53593262 & 3.42698188 \\
\hline $\mathrm{H}$ & -1.57122773 & -1.43161488 & 2.47932853 \\
\hline $\mathrm{H}$ & -2.82131213 & -0.79136204 & 3.54322827 \\
\hline $\mathrm{H}$ & 0.20001269 & 1.65231461 & 3.07203885 \\
\hline $\mathrm{H}$ & 0.83902121 & 0.38954440 & 2.01874292 \\
\hline $\mathrm{H}$ & 1.94709405 & 1.45820362 & 2.88546075 \\
\hline $\mathrm{C}$ & 0.03640952 & -1.43120366 & -2.66318456 \\
\hline $\mathrm{C}$ & 0.69053956 & -2.04052609 & -1.60786632 \\
\hline $\mathrm{H}$ & 0.24749818 & -2.95578439 & -1.21325627 \\
\hline $\mathrm{H}$ & 0.50658148 & -0.64777226 & -3.25238550 \\
\hline $\mathrm{H}$ & -0.82040605 & -1.92353606 & -3.11527204 \\
\hline $\mathrm{C}$ & 2.00843659 & -1.71315693 & -1.05737120 \\
\hline $\mathrm{C}$ & 2.53037253 & -2.53618433 & -0.04900580 \\
\hline $\mathrm{C}$ & 2.77305679 & -0.62138380 & -1.49935574 \\
\hline $\mathrm{C}$ & 3.77697100 & -2.28535499 & 0.50746263 \\
\hline $\mathrm{C}$ & 4.51317216 & -1.19330677 & 0.05554732 \\
\hline $\mathrm{C}$ & 4.01629190 & -0.36004819 & -0.94917698 \\
\hline $\mathrm{H}$ & 1.95588548 & -3.39123578 & 0.29845756 \\
\hline $\mathrm{H}$ & 2.39793631 & 0.03619469 & -2.27787665 \\
\hline $\mathrm{H}$ & 4.17794737 & -2.92952358 & 1.28186013 \\
\hline $\mathrm{H}$ & 4.60560945 & 0.48238865 & -1.29340154 \\
\hline $\mathrm{Br}$ & 6.19500931 & -0.83159384 & 0.80519295 \\
\hline
\end{tabular}


$\left[(\mathrm{NHC}) \mathrm{Au}\left(\mathrm{H}-\mathrm{C}_{8} \mathrm{H}_{7}\right)\right]^{+}$

C1 symmetry; $E=-1289.4822115$

\begin{tabular}{|c|c|c|c|}
\hline $\mathrm{Au}$ & 0.01407773 & -0.43402010 & -0.80630067 \\
\hline $\mathrm{C}$ & -0.88455776 & 0.96947767 & 0.33862204 \\
\hline $\mathrm{C}$ & -1.26568139 & 2.83507174 & 1.52527807 \\
\hline $\mathrm{C}$ & -2.40536392 & 2.09718379 & 1.54225560 \\
\hline $\mathrm{C}$ & 1.74148852 & 1.26124676 & 2.55916642 \\
\hline $\mathrm{C}$ & -2.28305589 & -1.17007386 & 2.72630635 \\
\hline $\mathrm{C}$ & -3.80505017 & 1.10671273 & -1.53556395 \\
\hline $\mathrm{C}$ & 0.09481466 & 3.79878919 & -1.50588163 \\
\hline $\mathrm{C}$ & 0.99987211 & 2.55802269 & 0.51063844 \\
\hline $\mathrm{C}$ & 1.21956230 & 3.38002563 & -0.60261369 \\
\hline $\mathrm{C}$ & 2.01933509 & 2.14827422 & 1.37937616 \\
\hline $\mathrm{C}$ & 2.53065384 & 3.79458107 & -0.84181277 \\
\hline $\mathrm{C}$ & 3.31328020 & 2.59058211 & 1.09812247 \\
\hline $\mathrm{C}$ & 3.56683491 & 3.40430367 & -0.00038367 \\
\hline $\mathrm{C}$ & -3.09804306 & -0.10066718 & 0.57778424 \\
\hline $\mathrm{C}$ & -3.91098735 & -0.03114648 & -0.56078046 \\
\hline $\mathrm{C}$ & -3.16839526 & -1.14130227 & 1.51301611 \\
\hline $\mathrm{C}$ & -4.82460135 & -1.06821060 & -0.75702443 \\
\hline $\mathrm{C}$ & -4.09784994 & -2.15439470 & 1.27221379 \\
\hline $\mathrm{C}$ & -4.91665162 & -2.11910798 & 0.14877514 \\
\hline $\mathrm{N}$ & -0.34490155 & 2.12571207 & 0.78242146 \\
\hline $\mathrm{N}$ & -2.15157989 & 0.95797871 & 0.80709224 \\
\hline $\mathrm{H}$ & -1.02713411 & 3.78873858 & 1.96983165 \\
\hline $\mathrm{H}$ & -3.36341358 & 2.27585735 & 2.00513742 \\
\hline $\mathrm{H}$ & -5.47257839 & -1.04287808 & -1.62836559 \\
\hline $\mathrm{H}$ & -4.18084519 & -2.97401740 & 1.98016504 \\
\hline $\mathrm{H}$ & -5.63740323 & -2.91376657 & -0.01861758 \\
\hline $\mathrm{H}$ & 2.73433077 & 4.43583713 & -1.69450146 \\
\hline $\mathrm{H}$ & 4.12555328 & 2.29660533 & 1.75667937 \\
\hline $\mathrm{H}$ & 4.57902066 & 3.74407390 & -0.19833031 \\
\hline $\mathrm{H}$ & -0.40050768 & 2.93236904 & -1.95823353 \\
\hline $\mathrm{H}$ & -0.67305547 & 4.36312241 & -0.96601902 \\
\hline $\mathrm{H}$ & 0.46584803 & 4.43227426 & -2.31375083 \\
\hline $\mathrm{H}$ & -3.93773300 & 2.07719677 & -1.04600087 \\
\hline $\mathrm{H}$ & -2.82463559 & 1.12337836 & -2.02524434 \\
\hline $\mathrm{H}$ & -4.56551827 & 1.01863905 & -2.31377376 \\
\hline $\mathrm{H}$ & -2.49137414 & -2.05334175 & 3.33314125 \\
\hline $\mathrm{H}$ & -1.22278174 & -1.19268456 & 2.45061526 \\
\hline $\mathrm{H}$ & -2.43182825 & -0.28884536 & 3.35953378 \\
\hline $\mathrm{H}$ & 0.92236335 & 1.64431539 & 3.17617122 \\
\hline $\mathrm{H}$ & 1.46236223 & 0.25084245 & 2.23850053 \\
\hline $\mathrm{H}$ & 2.62663888 & 1.17602380 & 3.19249417 \\
\hline $\mathrm{C}$ & 0.94029394 & -1.56961952 & -2.47333773 \\
\hline $\mathrm{C}$ & 1.07072715 & -2.41283769 & -1.38539355 \\
\hline $\mathrm{H}$ & 0.27376081 & -3.14053700 & -1.22619261 \\
\hline $\mathrm{H}$ & 1.76877694 & -0.96140144 & -2.82776738 \\
\hline $\mathrm{H}$ & 0.12265343 & -1.72283633 & -3.17270635 \\
\hline $\mathrm{C}$ & 2.23583181 & -2.58313538 & -0.51135737 \\
\hline $\mathrm{C}$ & 2.18183587 & -3.58401241 & 0.46957086 \\
\hline $\mathrm{C}$ & 3.40057158 & -1.80778017 & -0.63104456 \\
\hline $\mathrm{C}$ & 3.26589243 & -3.81177087 & 1.30899162 \\
\hline $\mathrm{C}$ & 4.41563619 & -3.03757075 & 1.17994228 \\
\hline $\mathrm{C}$ & 4.47971754 & -2.03579320 & 0.20920129 \\
\hline $\mathrm{H}$ & 1.28633752 & -4.19364342 & 0.56284279 \\
\hline $\mathrm{H}$ & 3.46599183 & -1.02722355 & -1.38346930 \\
\hline $\mathrm{H}$ & 3.21575507 & -4.59554737 & 2.05817442 \\
\hline $\mathrm{H}$ & 5.37927523 & -1.43702304 & 0.10561726 \\
\hline $\mathrm{H}$ & 5.26603312 & -3.21566313 & 1.83111507 \\
\hline
\end{tabular}


$\left[(\mathrm{NHC}) \mathrm{Au}\left(\mathrm{CH}_{3}-\mathrm{C}_{8} \mathrm{H}_{7}\right)\right]^{+}$

C1 symmetry; $\mathrm{E}=\mathbf{- 1 3 2 8 . 7 5 5 9 0 0 4}$

\begin{tabular}{|c|c|c|c|}
\hline $\mathrm{Au}$ & -0.12031397 & -0.29519274 & -0.88642749 \\
\hline $\mathrm{C}$ & -1.22489198 & 0.79680459 & 0.40811414 \\
\hline $\mathrm{C}$ & -1.93391398 & 2.45732220 & 1.74091242 \\
\hline $\mathrm{C}$ & -2.86932364 & 1.47408053 & 1.77669571 \\
\hline $\mathrm{C}$ & 1.42433203 & 1.57939801 & 2.47846871 \\
\hline $\mathrm{C}$ & -1.91794634 & -1.73447004 & 2.72327747 \\
\hline $\mathrm{C}$ & -4.22680335 & 0.35054739 & -1.27480737 \\
\hline $\mathrm{C}$ & -1.06464067 & 3.86790414 & -1.29515991 \\
\hline $\mathrm{C}$ & 0.25133305 & 2.77083719 & 0.57270958 \\
\hline $\mathrm{C}$ & 0.19130972 & 3.67804346 & -0.49341253 \\
\hline $\mathrm{C}$ & 1.40044394 & 2.56713955 & 1.34722002 \\
\hline $\mathrm{C}$ & 1.34983795 & 4.40006309 & -0.78409395 \\
\hline $\mathrm{C}$ & 2.53275553 & 3.31433621 & 1.01778403 \\
\hline $\mathrm{C}$ & 2.50857813 & 4.22046211 & -0.03646054 \\
\hline $\mathrm{C}$ & -3.11136483 & -0.76986056 & 0.70624665 \\
\hline $\mathrm{C}$ & -3.99988459 & -0.83047887 & -0.37491593 \\
\hline $\mathrm{C}$ & -2.87431630 & -1.84676761 & 1.57026511 \\
\hline $\mathrm{C}$ & -4.66551667 & -2.03898092 & -0.58734876 \\
\hline $\mathrm{C}$ & -3.56411516 & -3.03330806 & 1.31622230 \\
\hline $\mathrm{C}$ & -4.44963110 & -3.12909905 & 0.24829500 \\
\hline $\mathrm{N}$ & -0.93331568 & 2.02221468 & 0.89648186 \\
\hline $\mathrm{N}$ & -2.41686037 & 0.46565045 & 0.95108239 \\
\hline $\mathrm{H}$ & -1.88774097 & 3.41575447 & 2.23412039 \\
\hline $\mathrm{H}$ & -3.80539230 & 1.39965026 & 2.30796820 \\
\hline $\mathrm{H}$ & -5.36412256 & -2.11826139 & -1.41525578 \\
\hline $\mathrm{H}$ & -3.40614958 & -3.88626184 & 1.96998691 \\
\hline $\mathrm{H}$ & -4.98102179 & -4.05903046 & 0.06982040 \\
\hline $\mathrm{H}$ & 1.33546266 & 5.11420331 & -1.60236311 \\
\hline $\mathrm{H}$ & 3.43828212 & 3.18519337 & 1.60367668 \\
\hline $\mathrm{H}$ & 3.39753963 & 4.79751382 & -0.27289788 \\
\hline $\mathrm{H}$ & -1.38200988 & 2.93334680 & -1.77102295 \\
\hline $\mathrm{H}$ & -1.89764915 & 4.21129699 & -0.67244608 \\
\hline $\mathrm{H}$ & -0.91051738 & 4.60912131 & -2.08159850 \\
\hline $\mathrm{H}$ & -4.53687371 & 1.23801352 & -0.71324653 \\
\hline $\mathrm{H}$ & -3.31507557 & 0.61658120 & -1.82188660 \\
\hline $\mathrm{H}$ & -5.00457689 & 0.13106028 & -2.00869896 \\
\hline $\mathrm{H}$ & -1.87436592 & -2.67307241 & 3.27912952 \\
\hline $\mathrm{H}$ & -0.90395416 & -1.49638659 & 2.38293947 \\
\hline $\mathrm{H}$ & -2.21549407 & -0.94663175 & 3.42359746 \\
\hline $\mathrm{H}$ & 0.58490719 & 1.72342180 & 3.16618094 \\
\hline $\mathrm{H}$ & 1.36765662 & 0.54969839 & 2.10674256 \\
\hline $\mathrm{H}$ & 2.34810386 & 1.67519245 & 3.05241798 \\
\hline $\mathrm{C}$ & 0.92586587 & -1.10001007 & -2.66617276 \\
\hline $\mathrm{C}$ & 1.29357559 & -1.97406891 & -1.65818509 \\
\hline $\mathrm{H}$ & 0.66977359 & -2.85857735 & -1.52108090 \\
\hline $\mathrm{H}$ & 1.59201135 & -0.31170653 & -3.00803374 \\
\hline $\mathrm{H}$ & 0.12023015 & -1.37470873 & -3.34193445 \\
\hline $\mathrm{C}$ & 2.51528427 & -1.96112140 & -0.85454049 \\
\hline $\mathrm{C}$ & 2.72273270 & -3.00640224 & 0.05671917 \\
\hline $\mathrm{C}$ & 3.50054580 & -0.96595203 & -0.96737679 \\
\hline $\mathrm{C}$ & 3.87774664 & -3.06162963 & 0.82440554 \\
\hline $\mathrm{C}$ & 4.86338039 & -2.07686513 & 0.70977197 \\
\hline $\mathrm{C}$ & 4.64858004 & -1.02715853 & -0.19707727 \\
\hline $\mathrm{H}$ & 1.97262510 & -3.78763902 & 0.15479622 \\
\hline $\mathrm{H}$ & 3.37064961 & -0.14029365 & -1.66127445 \\
\hline $\mathrm{H}$ & 4.02143173 & -3.88403792 & 1.51968231 \\
\hline $\mathrm{H}$ & 5.40285960 & -0.25154115 & -0.29996248 \\
\hline $\mathrm{C}$ & 6.13056251 & -2.15144041 & 1.50750353 \\
\hline $\mathrm{H}$ & 6.47170895 & -1.15823530 & 1.81239676 \\
\hline $\mathrm{H}$ & 6.00754626 & -2.76500599 & 2.40311013 \\
\hline $\mathrm{H}$ & 6.93309791 & -2.59943721 & 0.90959904 \\
\hline
\end{tabular}


$\left[(\mathrm{NHC}) \mathrm{Au}\left(\mathrm{OMe}-\mathrm{C}_{8} \mathrm{H}_{7}\right)\right]^{+}$

C1 symmetry; $\mathrm{E}=\mathbf{- 1 4 0 3 . 8 8 7 5 0 2 4}$

\begin{tabular}{|c|c|c|c|}
\hline $\mathrm{Au}$ & -0.34406540 & -0.26692961 & -0.92617665 \\
\hline $\mathrm{C}$ & -1.45927915 & 0.69261858 & 0.46054066 \\
\hline $\mathrm{C}$ & -2.21374030 & 2.25368071 & 1.88683493 \\
\hline $\mathrm{C}$ & -3.07791082 & 1.20765547 & 1.92820652 \\
\hline $\mathrm{C}$ & 1.24029947 & 1.56320566 & 2.41007708 \\
\hline $\mathrm{C}$ & -1.88039060 & -1.95739036 & 2.71397517 \\
\hline $\mathrm{C}$ & -4.49152124 & 0.10031383 & -1.10725842 \\
\hline $\mathrm{C}$ & -1.60091180 & 3.84431806 & -1.11068937 \\
\hline $\mathrm{C}$ & -0.11441309 & 2.75764884 & 0.63087934 \\
\hline $\mathrm{C}$ & -0.29248792 & 3.70476965 & -0.38626133 \\
\hline $\mathrm{C}$ & 1.08792817 & 2.59672812 & 1.33099068 \\
\hline $\mathrm{C}$ & 0.79905669 & 4.51431612 & -0.70426012 \\
\hline $\mathrm{C}$ & 2.15000080 & 3.43062371 & 0.97653630 \\
\hline $\mathrm{C}$ & 2.00783162 & 4.37905090 & -0.03006286 \\
\hline $\mathrm{C}$ & -3.22129836 & -1.00817327 & 0.78492611 \\
\hline $\mathrm{C}$ & -4.15030408 & -1.09201939 & -0.25998114 \\
\hline $\mathrm{C}$ & -2.87737124 & -2.09512768 & 1.59879437 \\
\hline $\mathrm{C}$ & -4.74492620 & -2.33426563 & -0.48783142 \\
\hline $\mathrm{C}$ & -3.49914482 & -3.31576804 & 1.33137954 \\
\hline $\mathrm{C}$ & -4.42256620 & -3.43471665 & 0.29846283 \\
\hline $\mathrm{N}$ & -1.22832834 & 1.91811219 & 0.98113994 \\
\hline $\mathrm{N}$ & -2.59875267 & 0.26183517 & 1.04515112 \\
\hline $\mathrm{H}$ & -2.20813941 & 3.19516286 & 2.41364024 \\
\hline $\mathrm{H}$ & -3.98037090 & 1.05058453 & 2.49818596 \\
\hline $\mathrm{H}$ & -5.47219833 & -2.43228701 & -1.28860872 \\
\hline $\mathrm{H}$ & -3.25731179 & -4.17764140 & 1.94668523 \\
\hline $\mathrm{H}$ & -4.89966847 & -4.39148313 & 0.10877103 \\
\hline $\mathrm{H}$ & 0.69251551 & 5.26102209 & -1.48584552 \\
\hline $\mathrm{H}$ & 3.09432532 & 3.33491399 & 1.50477587 \\
\hline $\mathrm{H}$ & 2.84333155 & 5.02347429 & -0.28664459 \\
\hline $\mathrm{H}$ & -1.88500368 & 2.91044901 & -1.60842283 \\
\hline $\mathrm{H}$ & -2.41768060 & 4.10805262 & -0.43030395 \\
\hline $\mathrm{H}$ & -1.53788731 & 4.62437737 & -1.87174502 \\
\hline $\mathrm{H}$ & -4.82891464 & 0.94809181 & -0.50184957 \\
\hline $\mathrm{H}$ & -3.62417611 & 0.44028780 & -1.68472031 \\
\hline $\mathrm{H}$ & -5.28762805 & -0.14437983 & -1.81300257 \\
\hline $\mathrm{H}$ & -1.74466133 & -2.91089017 & 3.22810667 \\
\hline $\mathrm{H}$ & -0.90287125 & -1.63210750 & 2.34081278 \\
\hline $\mathrm{H}$ & -2.20169057 & -1.22082440 & 3.45828489 \\
\hline $\mathrm{H}$ & 0.42946971 & 1.61384096 & 3.14378257 \\
\hline $\mathrm{H}$ & 1.23736124 & 0.55038479 & 1.99059053 \\
\hline $\mathrm{H}$ & 2.18381157 & 1.70028580 & 2.94206223 \\
\hline $\mathrm{C}$ & 0.66199705 & -0.96844377 & -2.76138963 \\
\hline $\mathrm{C}$ & 1.24314231 & -1.76740696 & -1.78826977 \\
\hline $\mathrm{H}$ & 0.76336291 & -2.72731945 & -1.59173523 \\
\hline $\mathrm{H}$ & 1.18205560 & -0.10667024 & -3.17261376 \\
\hline $\mathrm{H}$ & -0.14387329 & -1.37468468 & -3.36698571 \\
\hline $\mathrm{C}$ & 2.50544557 & -1.56758982 & -1.09277458 \\
\hline $\mathrm{C}$ & 2.95819922 & -2.57099368 & -0.22465672 \\
\hline $\mathrm{C}$ & 3.31309528 & -0.42444755 & -1.26624986 \\
\hline $\mathrm{C}$ & 4.16865356 & -2.46519262 & 0.44520447 \\
\hline $\mathrm{C}$ & 4.95778045 & -1.32359962 & 0.25797489 \\
\hline $\mathrm{C}$ & 4.51309172 & -0.30220236 & -0.60377243 \\
\hline $\mathrm{H}$ & 2.35336359 & -3.46325721 & -0.08121751 \\
\hline $\mathrm{H}$ & 2.99525820 & 0.37556190 & -1.92849976 \\
\hline $\mathrm{H}$ & 4.49160693 & -3.26691496 & 1.09851087 \\
\hline $\mathrm{H}$ & 5.14558420 & 0.56950866 & -0.73413360 \\
\hline $\mathrm{O}$ & 6.14000790 & -1.10743526 & 0.84562850 \\
\hline $\mathrm{C}$ & 6.66757042 & -2.10502136 & 1.70355933 \\
\hline $\mathrm{H}$ & 7.62784837 & -1.72336186 & 2.04867062 \\
\hline $\mathrm{H}$ & 6.01286174 & -2.27463990 & 2.56607783 \\
\hline $\mathrm{H}$ & 6.82303068 & -3.04767265 & 1.16695352 \\
\hline
\end{tabular}


$\left[(\mathrm{NHC}) \mathrm{Au}\left(\mathrm{NH}_{2}-\mathrm{C}_{8} \mathrm{H}_{7}\right)\right]^{+}$

C1 symmetry; $\mathrm{E}=\mathbf{- 1 3 4 4 . 7 9 1 7 7 5 4}$

\begin{tabular}{|c|c|c|c|}
\hline $\mathrm{Au}$ & -0.09649628 & -0.24631776 & -0.90519086 \\
\hline $\mathrm{C}$ & -1.26446865 & 0.75116652 & 0.41068992 \\
\hline $\mathrm{C}$ & -2.09209670 & 2.35092766 & 1.75331235 \\
\hline $\mathrm{C}$ & -2.95465293 & 1.30387661 & 1.78342580 \\
\hline $\mathrm{C}$ & 1.30631523 & 1.72872235 & 2.50666090 \\
\hline $\mathrm{C}$ & -1.77468350 & -1.83431247 & 2.70976587 \\
\hline $\mathrm{C}$ & -4.22489850 & 0.10308763 & -1.27612247 \\
\hline $\mathrm{C}$ & -1.31098469 & 3.81311698 & -1.29554657 \\
\hline $\mathrm{C}$ & 0.06465433 & 2.82634253 & 0.58965462 \\
\hline $\mathrm{C}$ & -0.05344494 & 3.72445753 & -0.47904544 \\
\hline $\mathrm{C}$ & 1.21878891 & 2.71206709 & 1.37481142 \\
\hline $\mathrm{C}$ & 1.04908767 & 4.53293155 & -0.75995091 \\
\hline $\mathrm{C}$ & 2.29377599 & 3.54333484 & 1.05541000 \\
\hline $\mathrm{C}$ & 2.21058567 & 4.44398435 & -0.00058653 \\
\hline $\mathrm{C}$ & -3.03687483 & -0.94496681 & 0.70128196 \\
\hline $\mathrm{C}$ & -3.91940636 & -1.06278362 & -0.37994862 \\
\hline $\mathrm{C}$ & -2.72522669 & -2.00719094 & 1.55955523 \\
\hline $\mathrm{C}$ & -4.50059985 & -2.31323581 & -0.59735927 \\
\hline $\mathrm{C}$ & -3.33200817 & -3.23732272 & 1.30128880 \\
\hline $\mathrm{C}$ & -4.21000311 & -3.38938652 & 0.23366789 \\
\hline $\mathrm{N}$ & -1.06268849 & 1.99220072 & 0.90630567 \\
\hline $\mathrm{N}$ & -2.43058201 & 0.33435597 & 0.95229652 \\
\hline $\mathrm{H}$ & -2.11424898 & 3.30781204 & 2.25108136 \\
\hline $\mathrm{H}$ & -3.88330081 & 1.15992995 & 2.31335097 \\
\hline $\mathrm{H}$ & -5.19205730 & -2.43695432 & -1.42584247 \\
\hline $\mathrm{H}$ & -3.11477953 & -4.08017416 & 1.95120944 \\
\hline $\mathrm{H}$ & -4.67642946 & -4.35289065 & 0.05154283 \\
\hline $\mathrm{H}$ & 0.98822280 & 5.24225666 & -1.58028675 \\
\hline $\mathrm{H}$ & 3.20089462 & 3.48406055 & 1.65007365 \\
\hline $\mathrm{H}$ & 3.05496783 & 5.08724632 & -0.22956805 \\
\hline $\mathrm{H}$ & -1.53228395 & 2.86180089 & -1.79231368 \\
\hline $\mathrm{H}$ & -2.18059582 & 4.06477084 & -0.67921944 \\
\hline $\mathrm{H}$ & -1.21599184 & 4.57998799 & -2.06665687 \\
\hline $\mathrm{H}$ & -4.59210557 & 0.96657726 & -0.71166111 \\
\hline $\mathrm{H}$ & -3.33228955 & 0.42953781 & -1.82198648 \\
\hline $\mathrm{H}$ & -4.98680606 & -0.16516088 & -2.01051779 \\
\hline $\mathrm{H}$ & -1.67121028 & -2.76766169 & 3.26668080 \\
\hline $\mathrm{H}$ & -0.77899393 & -1.53409285 & 2.36451843 \\
\hline $\mathrm{H}$ & -2.11791656 & -1.06492703 & 3.40965190 \\
\hline $\mathrm{H}$ & 0.46381776 & 1.82530672 & 3.19906193 \\
\hline $\mathrm{H}$ & 1.30517501 & 0.69736407 & 2.13547158 \\
\hline $\mathrm{H}$ & 2.22638968 & 1.87792616 & 3.07520507 \\
\hline $\mathrm{C}$ & 1.02418004 & -1.01830334 & -2.62994992 \\
\hline $\mathrm{C}$ & 1.43074920 & -1.91637943 & -1.64898915 \\
\hline $\mathrm{H}$ & 0.81871595 & -2.81131406 & -1.52579752 \\
\hline $\mathrm{H}$ & 1.67779566 & -0.21624033 & -2.96471569 \\
\hline $\mathrm{H}$ & 0.25029204 & -1.32333108 & -3.32983906 \\
\hline $\mathrm{C}$ & 2.63189470 & -1.89535745 & -0.84420129 \\
\hline $\mathrm{C}$ & 2.85537117 & -2.94257609 & 0.07033926 \\
\hline $\mathrm{C}$ & 3.61924649 & -0.89391270 & -0.94603971 \\
\hline $\mathrm{C}$ & 3.99775087 & -3.00199436 & 0.83978117 \\
\hline $\mathrm{C}$ & 4.98277408 & -1.99917038 & 0.72746940 \\
\hline $\mathrm{C}$ & 4.76354288 & -0.93914230 & -0.18326710 \\
\hline $\mathrm{H}$ & 2.11363911 & -3.73244453 & 0.16326173 \\
\hline $\mathrm{H}$ & 3.48895794 & -0.06997494 & -1.64179053 \\
\hline $\mathrm{H}$ & 4.14850079 & -3.82668037 & 1.53050547 \\
\hline $\mathrm{H}$ & 5.51442731 & -0.16030704 & -0.28374451 \\
\hline $\mathrm{N}$ & 6.10409211 & -2.03386548 & 1.48893596 \\
\hline $\mathrm{H}$ & 6.32364288 & -2.85505706 & 2.02631133 \\
\hline $\mathrm{H}$ & 6.86481468 & -1.40498586 & 1.29667891 \\
\hline
\end{tabular}


$\left[\mathrm{Au}\left(\mathrm{NO}_{2}-\mathrm{NHC}\right)\right]^{+}$

Cs symmetry; $\mathrm{E}=\mathbf{- 1 1 8 4 . 3 9 9 3 9 2 9}$

\begin{tabular}{|c|c|c|c|}
\hline $\mathrm{Au}$ & -1.78965150 & 0.35920941 & 0.00000000 \\
\hline $\mathrm{C}$ & 0.17062716 & 0.12714217 & 0.00000000 \\
\hline $\mathrm{C}$ & 2.35034986 & 0.56378715 & 0.00000000 \\
\hline $\mathrm{C}$ & 2.17587627 & -0.78523640 & 0.00000000 \\
\hline $\mathrm{C}$ & 0.84208311 & 2.43788988 & -2.53760564 \\
\hline $\mathrm{C}$ & 0.24603014 & -2.28159748 & -2.53389910 \\
\hline $\mathrm{C}$ & 0.24603014 & -2.28159748 & 2.53389910 \\
\hline $\mathrm{C}$ & 0.84208311 & 2.43788988 & 2.53760564 \\
\hline $\mathrm{C}$ & 0.80149204 & 2.52531275 & 0.00000000 \\
\hline $\mathrm{C}$ & 0.67503043 & 3.17099324 & 1.23700127 \\
\hline $\mathrm{C}$ & 0.67503043 & 3.17099324 & -1.23700127 \\
\hline $\mathrm{C}$ & 0.39157920 & 4.53724227 & 1.20785827 \\
\hline $\mathrm{C}$ & 0.39157920 & 4.53724227 & -1.20785827 \\
\hline $\mathrm{C}$ & 0.24989555 & 5.21169811 & 0.00000000 \\
\hline $\mathrm{C}$ & 0.13207956 & -2.32629370 & 0.00000000 \\
\hline $\mathrm{C}$ & -0.19159531 & -2.89658735 & 1.23617732 \\
\hline $\mathrm{C}$ & -0.19159531 & -2.89658735 & -1.23617732 \\
\hline $\mathrm{C}$ & -0.90760280 & -4.09396509 & 1.20737815 \\
\hline $\mathrm{C}$ & -0.90760280 & -4.09396509 & -1.20737815 \\
\hline $\mathrm{C}$ & -1.26556335 & -4.68362726 & 0.00000000 \\
\hline $\mathrm{N}$ & 1.09591579 & 1.11228727 & 0.00000000 \\
\hline $\mathrm{N}$ & 0.81941545 & -1.05047286 & 0.00000000 \\
\hline $\mathrm{H}$ & 3.25633282 & 1.15011117 & 0.00000000 \\
\hline $\mathrm{H}$ & -1.17584940 & -4.56964961 & 2.14600775 \\
\hline $\mathrm{H}$ & -1.17584940 & -4.56964961 & -2.14600775 \\
\hline $\mathrm{H}$ & -1.81992345 & -5.61701139 & 0.00000000 \\
\hline $\mathrm{H}$ & 0.28769265 & 5.07415926 & 2.14601236 \\
\hline $\mathrm{H}$ & 0.28769265 & 5.07415926 & -2.14601236 \\
\hline $\mathrm{H}$ & 0.03299257 & 6.27544579 & 0.00000000 \\
\hline $\mathrm{H}$ & 0.11265680 & 1.62699312 & 2.64573074 \\
\hline $\mathrm{H}$ & 1.83932333 & 1.99412034 & 2.63153680 \\
\hline $\mathrm{H}$ & 0.70544435 & 3.11791086 & 3.38013670 \\
\hline $\mathrm{H}$ & 1.33568498 & -2.33025816 & 2.64210517 \\
\hline $\mathrm{H}$ & -0.04912002 & -1.22995793 & 2.61933362 \\
\hline $\mathrm{H}$ & -0.18967676 & -2.81731456 & 3.37907393 \\
\hline $\mathrm{H}$ & -0.18967676 & -2.81731456 & -3.37907393 \\
\hline $\mathrm{H}$ & -0.04912002 & -1.22995793 & -2.61933362 \\
\hline $\mathrm{H}$ & 1.33568498 & -2.33025816 & -2.64210517 \\
\hline $\mathrm{H}$ & 1.83932333 & 1.99412034 & -2.63153680 \\
\hline $\mathrm{H}$ & 0.11265680 & 1.62699312 & -2.64573074 \\
\hline $\mathrm{H}$ & 0.70544435 & 3.11791086 & -3.38013670 \\
\hline $\mathrm{N}$ & 3.21903982 & -1.77584570 & 0.00000000 \\
\hline $\mathrm{O}$ & 2.88451166 & -2.94496104 & 0.00000000 \\
\hline $\mathrm{O}$ & 4.35115302 & -1.33057387 & 0.00000000 \\
\hline
\end{tabular}


$\left[\mathrm{Au}\left(\mathrm{CF}_{3}-\mathrm{NHC}\right)\right]^{+}$

Cs symmetry; $\mathrm{E}=\mathbf{- 1 3 1 6 . 8 4 5 2 5 4 4}$

$\begin{array}{lccc}\mathrm{Au} & -1.88036147 & 0.67609670 & 0.00000000 \\ \mathrm{C} & 0.03966652 & 0.22164036 & 0.00000000 \\ \mathrm{C} & 2.25434583 & 0.39480765 & 0.00000000 \\ \mathrm{C} & 1.93829494 & -0.92650533 & 0.00000000 \\ \mathrm{C} & 0.96999243 & 2.43727686 & -2.53660048 \\ \mathrm{C} & -0.17748823 & -2.15744500 & -2.53882214 \\ \mathrm{C} & -0.17748823 & -2.15744500 & 2.53882214 \\ \mathrm{C} & 0.96999243 & 2.43727686 & 2.53660048 \\ \mathrm{C} & 0.94303390 & 2.52785035 & 0.00000000 \\ \mathrm{C} & 0.89402390 & 3.18556044 & 1.23616481 \\ \mathrm{C} & 0.89402390 & 3.18556044 & -1.23616481 \\ \mathrm{C} & 0.77536441 & 4.57590273 & 1.20763302 \\ \mathrm{C} & 0.77536441 & 4.57590273 & -1.20763302 \\ \mathrm{C} & 0.71494734 & 5.26275402 & 0.00000000 \\ \mathrm{C} & -0.23118415 & -2.22968303 & 0.00000000 \\ \mathrm{C} & -0.59830437 & -2.77680853 & 1.23694976 \\ \mathrm{C} & -0.59830437 & -2.77680853 & -1.23694976 \\ \mathrm{C} & -1.37411934 & -3.93651519 & 1.20743089 \\ \mathrm{C} & -1.37411934 & -3.93651519 & -1.20743089 \\ \mathrm{C} & -1.75658105 & -4.51023006 & 0.00000000 \\ \mathrm{~N} & 1.06800322 & 1.09115521 & 0.00000000 \\ \mathrm{~N} & 0.55770942 & -1.01913820 & 0.00000000 \\ \mathrm{H} & 3.21312921 & 0.88995325 & 0.00000000 \\ \mathrm{H} & -1.67507298 & -4.39258681 & 2.14586373 \\ \mathrm{H} & -1.67507298 & -4.39258681 & -2.14586373 \\ \mathrm{H} & -2.35702477 & -5.41476302 & 0.00000000 \\ \mathrm{H} & 0.73590683 & 5.12115065 & 2.14595981 \\ \mathrm{H} & 0.73590683 & 5.12115065 & -2.14595981 \\ \mathrm{H} & 0.62617196 & 6.34478399 & 0.00000000 \\ \mathrm{H} & 0.14054533 & 1.72962004 & 2.64804597 \\ \mathrm{~F} & 1.89941098 & 1.86420486 & 2.62431056 \\ \mathrm{H} & 0.92893623 & 3.12931219 & 3.37950411 \\ \mathrm{H} & 0.91106927 & -2.16480905 & 2.64992952 \\ \mathrm{H} & -0.51213197 & -1.11734031 & 2.62412623 \\ \mathrm{H} & -0.60079573 & -2.71038127 & 3.37919974 \\ \mathrm{H} & -0.60079573 & -2.71038127 & -3.37919974 \\ \mathrm{H} & -0.51213197 & -1.11734031 & -2.62412623 \\ \mathrm{H} & 0.91106927 & -2.16480905 & -2.64992952 \\ \mathrm{H} & 1.89941098 & 1.86420486 & -2.62431056 \\ \mathrm{H} & 0.14054533 & 1.72962004 & -2.64804597 \\ \mathrm{H} & 0.92893623 & 3.12931219 & -3.37950411 \\ & 2.86924446 & -2.09901058 & 0.00000000 \\ \mathrm{H} & 2.68935934 & -1.64988163 & 0.00000000 \\ & & -86614082 & -1.07883400 \\ & & -2.86614082 & 1.07883400\end{array}$


$[\mathrm{Au}(\mathrm{Br}-\mathrm{NHC})]^{+}$

Cs symmetry; $\mathrm{E}=\mathbf{- 3 5 5 0 . 5 9 7 4 4 4 6}$

$\begin{array}{lccc}\mathrm{Au} & -1.88089018 & 0.76083658 & 0.00000000 \\ \mathrm{C} & 0.02354065 & 0.24656447 & 0.00000000 \\ \mathrm{C} & 2.24705722 & 0.35106412 & 0.00000000 \\ \mathrm{C} & 1.88948515 & -0.96129484 & 0.00000000 \\ \mathrm{C} & 1.01956399 & 2.42908782 & -2.53620250 \\ \mathrm{C} & -0.21447354 & -2.15075675 & -2.53369221 \\ \mathrm{C} & -0.21447354 & -2.15075675 & 2.53369221 \\ \mathrm{C} & 1.01956399 & 2.42908782 & 2.53620250 \\ \mathrm{C} & 0.99711995 & 2.52005006 & 0.00000000 \\ \mathrm{C} & 0.97024764 & 3.17968581 & 1.23588708 \\ \mathrm{C} & 0.97024764 & 3.17968581 & -1.23588708 \\ \mathrm{C} & 0.89698993 & 4.57316517 & 1.20755092 \\ \mathrm{C} & 0.89698993 & 4.57316517 & -1.20755092 \\ \mathrm{C} & 0.85896880 & 5.26173904 & 0.00000000 \\ \mathrm{C} & -0.30570000 & -2.20325027 & 0.00000000 \\ \mathrm{C} & -0.67440117 & -2.75003203 & 1.23589947 \\ \mathrm{C} & -0.67440117 & -2.75003203 & -1.23589947 \\ \mathrm{C} & -1.47463259 & -3.89294198 & 1.20779240 \\ \mathrm{C} & -1.47463259 & -3.89294198 & -1.20779240 \\ \mathrm{C} & -1.87293109 & -4.45564070 & 0.00000000 \\ \mathrm{~N} & 1.07615354 & 1.08098521 & 0.00000000 \\ \mathrm{~N} & 0.50516265 & -1.01233921 & 0.00000000 \\ \mathrm{H} & 3.21893099 & 0.81843673 & 0.00000000 \\ \mathrm{H} & -1.78021299 & -4.34616935 & 2.14617626 \\ \mathrm{H} & -1.78021299 & -4.34616935 & -2.14617626 \\ \mathrm{H} & -2.49375285 & -5.34634919 & 0.00000000 \\ \mathrm{H} & 0.87558345 & 5.11933877 & 2.14595683 \\ \mathrm{H} & 0.87558345 & 5.11933877 & -2.14595683 \\ \mathrm{H} & 0.80588876 & 6.34612604 & 0.00000000 \\ \mathrm{H} & 0.15247735 & 1.76961304 & 2.65661764 \\ \mathrm{H} & 1.91547585 & 1.80379569 & 2.61306710 \\ \mathrm{H} & 1.02666131 & 3.12264815 & 3.37886816 \\ \mathrm{H} & 0.87694703 & -2.18771318 & 2.62508784 \\ \mathrm{H} & -0.51600174 & -1.10187451 & 2.63143220 \\ \mathrm{H} & -0.63540476 & -2.69744749 & 3.37939363 \\ \mathrm{H} & -0.63540476 & -2.69744749 & -3.37939363 \\ \mathrm{H} & -0.51600174 & -1.10187451 & -2.63143220 \\ \mathrm{H} & 0.87694703 & -2.18771318 & -2.62508784 \\ \mathrm{H} & 1.91547585 & 1.80379569 & -2.61306710 \\ \mathrm{H} & 0.15247735 & 1.76961304 & -2.65661764 \\ \mathrm{Br} & 1.02666131 & 3.12264815 & -3.37886816 \\ & 2.93869194 & -2.47380272 & 0.00000000\end{array}$


[Au(H-NHC) $]^{+}$

C2v symmetry; $\mathrm{E}=\mathbf{- 9 8 0 . 1 3 0 3 2 9}$

$\begin{array}{lrrr}\mathrm{Au} & 0.00000000 & -0.00000000 & 1.49863164 \\ \mathrm{C} & -0.00000000 & 0.00000000 & -0.47373928 \\ \mathrm{C} & -0.00000000 & 0.67906823 & -2.59216415 \\ \mathrm{C} & -0.00000000 & -0.67906823 & -2.59216415 \\ \mathrm{C} & -2.53583248 & 2.36905835 & -0.86219743 \\ \mathrm{C} & -2.53583248 & -2.36905835 & -0.86219743 \\ \mathrm{C} & 2.53583248 & -2.36905835 & -0.86219743 \\ \mathrm{C} & 2.53583248 & 2.36905835 & -0.86219743 \\ \mathrm{C} & 0.00000000 & 2.45040889 & -0.81844042 \\ \mathrm{C} & 1.23537293 & 3.08063322 & -0.61881095 \\ \mathrm{C} & -1.23537293 & 3.08063322 & -0.61881095 \\ \mathrm{C} & 1.20736703 & 4.40656555 & -0.18383844 \\ \mathrm{C} & -1.20736703 & 4.40656555 & -0.18383844 \\ \mathrm{C} & 0.00000000 & 5.06150961 & 0.03283637 \\ \mathrm{C} & -0.00000000 & -2.45040889 & -0.81844042 \\ \mathrm{C} & 1.23537293 & -3.08063322 & -0.61881095 \\ \mathrm{C} & -1.23537293 & -3.08063322 & -0.61881095 \\ \mathrm{C} & 1.20736703 & -4.40656555 & -0.18383844 \\ \mathrm{C} & -1.20736703 & -4.40656555 & -0.18383844 \\ \mathrm{C} & -0.00000000 & -5.06150961 & 0.03283637 \\ \mathrm{~N} & -0.00000000 & 1.08273214 & -1.27199611 \\ \mathrm{~N} & -0.00000000 & -1.08273214 & -1.27199611 \\ \mathrm{H} & -0.00000000 & 1.39275826 & -3.40140886 \\ \mathrm{H} & -0.00000000 & -1.39275826 & -3.40140886 \\ \mathrm{H} & 2.14587746 & -4.92790879 & -0.02006065 \\ \mathrm{H} & -2.14587746 & -4.92790879 & -0.02006065 \\ \mathrm{H} & -0.00000000 & -6.09424767 & 0.36776361 \\ \mathrm{H} & 2.14587746 & 4.92790879 & -0.02006065 \\ \mathrm{H} & -2.14587746 & 4.92790879 & -0.02006065 \\ \mathrm{H} & 0.00000000 & 6.09424767 & 0.36776361 \\ \mathrm{H} & 2.65317900 & 1.50183347 & -0.20239035 \\ \mathrm{H} & 2.61523377 & 2.00507482 & -1.89213838 \\ \mathrm{H} & 3.37867320 & 3.03855910 & -0.68158208 \\ \mathrm{H} & 2.61523377 & -2.00507482 & -1.89213838 \\ \mathrm{H} & 2.65317900 & -1.50183347 & -0.20239035 \\ \mathrm{H} & 3.37867320 & -3.03855910 & -0.68158208 \\ \mathrm{H} & -3.37867320 & -3.03855910 & -0.68158208 \\ \mathrm{H} & -2.65317900 & -1.50183347 & -0.20239035 \\ \mathrm{H} & -2.61523377 & -2.00507482 & -1.89213838 \\ \mathrm{H} & -2.61523377 & 2.00507482 & -1.89213838 \\ \mathrm{H} & -2.65317900 & 1.50183347 & -0.20239035 \\ & -3.37867320 & 3.03855910 & -0.68158208\end{array}$


$\left[\mathrm{Au}\left(\mathrm{CH}_{3}-\mathrm{NHC}\right)\right]^{+}$

Cs symmetry; $\mathrm{E}=\mathbf{- 1 0 1 9 . 4 0 8 6 0 1 9}$

\begin{tabular}{|c|c|c|c|}
\hline $\mathrm{Au}$ & -1.62144542 & 0.14003909 & 0.00000000 \\
\hline $\mathrm{C}$ & 0.34826042 & 0.04242037 & 0.00000000 \\
\hline $\mathrm{C}$ & 2.49475808 & 0.61332531 & 0.00000000 \\
\hline $\mathrm{C}$ & 2.44531482 & -0.74804550 & 0.00000000 \\
\hline $\mathrm{C}$ & 0.84943640 & 2.38785346 & -2.53533435 \\
\hline $\mathrm{C}$ & 0.59736696 & -2.33389160 & -2.53533130 \\
\hline $\mathrm{C}$ & 0.59736696 & -2.33389160 & 2.53533130 \\
\hline $\mathrm{C}$ & 0.84943640 & 2.38785346 & 2.53533435 \\
\hline $\mathrm{C}$ & 0.81030542 & 2.47060906 & 0.00000000 \\
\hline $\mathrm{C}$ & 0.64240735 & 3.11091929 & 1.23494284 \\
\hline $\mathrm{C}$ & 0.64240735 & 3.11091929 & -1.23494284 \\
\hline $\mathrm{C}$ & 0.27392490 & 4.45686891 & 1.20724126 \\
\hline $\mathrm{C}$ & 0.27392490 & 4.45686891 & -1.20724126 \\
\hline $\mathrm{C}$ & 0.08998165 & 5.12191946 & 0.00000000 \\
\hline $\mathrm{C}$ & 0.56668695 & -2.41704923 & 0.00000000 \\
\hline $\mathrm{C}$ & 0.33044621 & -3.03673769 & 1.23455570 \\
\hline $\mathrm{C}$ & 0.33044621 & -3.03673769 & -1.23455570 \\
\hline $\mathrm{C}$ & -0.17397931 & -4.33790444 & 1.20724612 \\
\hline $\mathrm{C}$ & -0.17397931 & -4.33790444 & -1.20724612 \\
\hline $\mathrm{C}$ & -0.42383586 & -4.98122716 & 0.00000000 \\
\hline $\mathrm{N}$ & 1.19532279 & 1.08313098 & 0.00000000 \\
\hline $\mathrm{N}$ & 1.09464954 & -1.07810403 & 0.00000000 \\
\hline $\mathrm{H}$ & 3.33662109 & 1.28845250 & 0.00000000 \\
\hline $\mathrm{H}$ & -0.36769546 & -4.84885903 & 2.14583034 \\
\hline $\mathrm{H}$ & -0.36769546 & -4.84885903 & -2.14583034 \\
\hline $\mathrm{H}$ & -0.81287699 & -5.99483273 & 0.00000000 \\
\hline $\mathrm{H}$ & 0.13637721 & 4.98564093 & 2.14584164 \\
\hline $\mathrm{H}$ & 0.13637721 & 4.98564093 & -2.14584164 \\
\hline $\mathrm{H}$ & -0.19313960 & 6.17006168 & 0.00000000 \\
\hline $\mathrm{H}$ & 0.13989702 & 1.56132783 & 2.65613247 \\
\hline $\mathrm{H}$ & 1.85618612 & 1.96317662 & 2.60952372 \\
\hline $\mathrm{H}$ & 0.71258158 & 3.06769109 & 3.37819310 \\
\hline $\mathrm{H}$ & 1.63285575 & -1.98480393 & 2.61056439 \\
\hline $\mathrm{H}$ & -0.04695734 & -1.45569483 & 2.65795751 \\
\hline $\mathrm{H}$ & 0.41040952 & -3.00179915 & 3.37815240 \\
\hline $\mathrm{H}$ & 0.41040952 & -3.00179915 & -3.37815240 \\
\hline $\mathrm{H}$ & -0.04695734 & -1.45569483 & -2.65795751 \\
\hline $\mathrm{H}$ & 1.63285575 & -1.98480393 & -2.61056439 \\
\hline $\mathrm{H}$ & 1.85618612 & 1.96317662 & -2.60952372 \\
\hline $\mathrm{H}$ & 0.13989702 & 1.56132783 & -2.65613247 \\
\hline $\mathrm{H}$ & 0.71258158 & 3.06769109 & -3.37819310 \\
\hline $\mathrm{C}$ & 3.52314676 & -1.76822464 & 0.00000000 \\
\hline $\mathrm{H}$ & 4.49738149 & -1.27674653 & 0.00000000 \\
\hline $\mathrm{H}$ & 3.46307345 & -2.41311589 & 0.88215390 \\
\hline $\mathrm{H}$ & 3.46307345 & -2.41311589 & -0.88215390 \\
\hline
\end{tabular}


$\left[\mathrm{Au}\left(\mathrm{OCH}_{3}-\mathrm{NHC}\right)\right]^{+}$

Cs symmetry; $\mathrm{E}=\mathbf{- 1 0 9 4 . 5 3 0 3 7 5 7}$

$\begin{array}{lrrr}\mathrm{Au} & -1.76829290 & 0.24718801 & 0.00000000 \\ \mathrm{C} & 0.19610870 & 0.07741928 & 0.00000000 \\ \mathrm{C} & 2.37645415 & 0.56007997 & 0.00000000 \\ \mathrm{C} & 2.24212440 & -0.80010947 & 0.00000000 \\ \mathrm{C} & 0.79304974 & 2.39145323 & -2.53531500 \\ \mathrm{C} & 0.39477494 & -2.33759606 & -2.53176652 \\ \mathrm{C} & 0.39477494 & -2.33759606 & 2.53176652 \\ \mathrm{C} & 0.79304974 & 2.39145323 & 2.53531500 \\ \mathrm{C} & 0.76433424 & 2.47739335 & 0.00000000 \\ \mathrm{C} & 0.62613096 & 3.12475299 & 1.23493333 \\ \mathrm{C} & 0.62613096 & 3.12475299 & -1.23493333 \\ \mathrm{C} & 0.32033602 & 4.48632002 & 1.20726961 \\ \mathrm{C} & 0.32033602 & 4.48632002 & -1.20726961 \\ \mathrm{C} & 0.16743896 & 5.15915646 & 0.00000000 \\ \mathrm{C} & 0.30933077 & -2.40172397 & 0.00000000 \\ \mathrm{C} & 0.04635160 & -3.00858724 & 1.23438825 \\ \mathrm{C} & 0.04635160 & -3.00858724 & -1.23438825 \\ \mathrm{C} & -0.53736590 & -4.27584290 & 1.20746702 \\ \mathrm{C} & -0.53736590 & -4.27584290 & -1.20746702 \\ \mathrm{C} & -0.82976062 & -4.90066229 & 0.00000000 \\ \mathrm{~N} & 1.08649059 & 1.07439267 & 0.00000000 \\ \mathrm{~N} & 0.88900680 & -1.08380509 & 0.00000000 \\ \mathrm{H} & 3.24306473 & 1.20002926 & 0.00000000 \\ \mathrm{H} & -0.75665079 & -4.77627429 & 2.14607058 \\ \mathrm{H} & -0.75665079 & -4.77627429 & -2.14607058 \\ \mathrm{H} & -1.28239363 & -5.88756289 & 0.00000000 \\ \mathrm{H} & 0.20732755 & 5.02089280 & 2.14588056 \\ \mathrm{H} & 0.20732755 & 5.02089280 & -2.14588056 \\ \mathrm{H} & -0.06637826 & 6.21939999 & 0.00000000 \\ \mathrm{H} & 0.00767689 & 1.64005885 & 2.67705002 \\ \mathrm{H} & 1.75376752 & 1.86899606 & 2.58923066 \\ \mathrm{H} & 0.74216046 & 3.08520007 & 3.37645230 \\ \mathrm{H} & 1.47648122 & -2.18839573 & 2.62218994 \\ \mathrm{H} & -0.08060800 & -1.35505607 & 2.62662381 \\ \mathrm{H} & 0.07278794 & -2.94653586 & 3.37853562 \\ \mathrm{H} & 0.07278794 & -2.94653586 & -3.37853562 \\ \mathrm{H} & -0.08060800 & -1.35505607 & -2.62662381 \\ \mathrm{H} & 1.47648122 & -2.18839573 & -2.62218994 \\ \mathrm{H} & 1.75376752 & 1.86899606 & -2.58923066 \\ \mathrm{H} & 0.00767689 & 1.64005885 & -2.67705002 \\ \mathrm{H} & 0.74216046 & 3.08520007 & -3.37645230 \\ \mathrm{H} & 3.09304939 & -1.81010457 & 0.00000000 \\ \mathrm{H} & 5.47357064 & -1.44719198 & 0.00000000 \\ \mathrm{H} & 4.71813865 & -0.86985363 & -0.89791681 \\ \mathrm{H} & & -2.38322863 & 0.00000000 \\ \mathrm{H} & & & 0.89791681\end{array}$


$\left[\mathrm{Au}\left(\mathrm{NH}_{2}-\mathrm{NHC}\right)\right]^{+}$

C1 symmetry; $\mathrm{E}=\mathbf{- 1 0 3 5 . 4 3 3 6 8 6 4}$

$\begin{array}{lrrr}\mathrm{Au} & -0.14728431 & -1.61773912 & -0.17673676 \\ \mathrm{C} & -0.04793642 & 0.33863128 & 0.03933463 \\ \mathrm{C} & -0.61456395 & 2.48452452 & 0.26598577 \\ \mathrm{C} & 0.75380443 & 2.42018426 & 0.25472671 \\ \mathrm{C} & -2.37001723 & 1.11543558 & -2.43590873 \\ \mathrm{C} & 2.43515920 & 1.08284191 & -2.40743271 \\ \mathrm{C} & 2.26641871 & 0.16909065 & 2.57454285 \\ \mathrm{C} & -2.39369465 & 0.57298399 & 2.60480609 \\ \mathrm{C} & -2.46602288 & 0.80955901 & 0.08044071 \\ \mathrm{C} & -3.11358766 & 0.51560848 & 1.28761994 \\ \mathrm{C} & -3.10212678 & 0.78079612 & -1.16764386 \\ \mathrm{C} & -4.46190519 & 0.16226743 & 1.21564622 \\ \mathrm{C} & -4.45084085 & 0.42166671 & -1.18476086 \\ \mathrm{C} & -5.12270774 & 0.11382939 & -0.00691471 \\ \mathrm{C} & 2.42624714 & 0.57028848 & 0.07331183 \\ \mathrm{C} & 3.00998190 & 0.12655799 & 1.26941116 \\ \mathrm{C} & 3.09179044 & 0.56348769 & -1.16125654 \\ \mathrm{C} & 4.31554864 & -0.36251828 & 1.19771016 \\ \mathrm{C} & 4.39477281 & 0.06369624 & -1.17884969 \\ \mathrm{C} & 4.99837349 & -0.39675064 & -0.01344448 \\ \mathrm{~N} & -1.07690477 & 1.18371146 & 0.12662007 \\ \mathrm{~N} & 1.08382484 & 1.08079417 & 0.11714382 \\ \mathrm{H} & -1.29026639 & 3.32023435 & 0.34621121 \\ \mathrm{H} & 4.79835002 & -0.71518069 & 2.10441247 \\ \mathrm{H} & 4.93885825 & 0.04129109 & -2.11848231 \\ \mathrm{H} & 6.01375288 & -0.77977830 & -0.04811207 \\ \mathrm{H} & -4.99603146 & -0.07058644 & 2.13218922 \\ \mathrm{H} & -4.97636881 & 0.39043232 & -2.13470803 \\ \mathrm{H} & -6.17301348 & -0.15909054 & -0.04125689 \\ \mathrm{H} & -1.61026270 & -0.19091349 & 2.66858275 \\ \mathrm{H} & -1.91254784 & 1.54360299 & 2.76360916 \\ \mathrm{H} & -3.08834932 & 0.40455347 & 3.42973642 \\ \mathrm{H} & 1.86374099 & 1.16551740 & 2.78583684 \\ \mathrm{H} & 1.41909746 & -0.52606471 & 2.57767294 \\ \mathrm{H} & 2.92435916 & -0.10703412 & 3.40062201 \\ \mathrm{H} & 3.08429256 & 0.93931472 & -3.27311488 \\ \mathrm{H} & 1.48753492 & 0.57157738 & -2.60902464 \\ \mathrm{H} & 2.21785215 & 2.15349849 & -2.32342748 \\ \mathrm{H} & -1.87848326 & 2.09199247 & -2.37764287 \\ \mathrm{H} & -1.59307070 & 0.37492676 & -2.65849287 \\ \mathrm{H} & -3.05867820 & 1.13677884 & -3.28249137 \\ \mathrm{H} & 1.72134015 & 3.38058862 & 0.27034540 \\ & 1.44020962 & 4.29528694 & 0.58603097 \\ & 2.63450229 & 3.09228652 & 0.59134137\end{array}$


$\mathrm{NO}_{2}-\mathrm{C}_{2} \mathrm{H}_{3}$

C2 symmetry; $\mathrm{E}=\mathbf{- 2 8 2 . 7 7 9 8 0 6 2}$

$\begin{array}{lrrr}\mathrm{C} & -0.60389637 & -0.76823629 & 0.00000000 \\ \mathrm{C} & 0.15481759 & -1.85363798 & 0.00000000 \\ \mathrm{H} & -0.29731429 & -2.83883018 & 0.00000000 \\ \mathrm{H} & 1.23579965 & -1.76332300 & 0.00000000 \\ \mathrm{H} & -1.68493607 & -0.72409757 & 0.00000000 \\ \mathrm{~N} & 0.00000000 & 0.55852180 & 0.00000000 \\ \mathrm{O} & -0.78546437 & 1.49356705 & 0.00000000 \\ \mathrm{O} & 1.21557980 & 0.64991342 & 0.00000000\end{array}$

$\mathrm{CF}_{3}-\mathrm{C}_{2} \mathrm{H}_{3}$

Cs symmetry; $\mathrm{E}=\mathbf{- 4 1 5 . 2 1 1 2 1 8 9}$

$\begin{array}{lrrr}\text { C } & 0.52608608 & -1.04948714 & 0.00000000 \\ \text { C } & -0.30955045 & -2.08007845 & 0.00000000 \\ \text { H } & 0.05778995 & -3.10090931 & 0.00000000 \\ \text { H } & -1.38469132 & -1.93240193 & 0.00000000 \\ \text { H } & 1.60543897 & -1.17123967 & 0.00000000 \\ \text { C } & 0.06480413 & 0.36980129 & 0.00000000 \\ \text { F } & -1.27068062 & 0.47921088 & 0.00000000 \\ \text { F } & 0.52608608 & 1.02501326 & 1.07962340 \\ \text { F } & 0.52608608 & 1.02501326 & -1.07962340\end{array}$

$\mathrm{Br}-\mathrm{C}_{2} \mathrm{H}_{3}$

Cs symmetry; $\mathrm{E}=\mathbf{- 2 6 4 8 . 9 6 2 9 9 0 7}$

$\begin{array}{lrrc}\mathrm{C} & -0.44242941 & -2.07728081 & 0.00000000 \\ \mathrm{C} & 0.45766054 & -1.10508673 & 0.00000000 \\ \mathrm{H} & -1.50915804 & -1.88232459 & 0.00000000 \\ \mathrm{H} & -0.11134692 & -3.11165895 & 0.00000000 \\ \mathrm{H} & 1.52911821 & -1.26718653 & 0.00000000 \\ \mathrm{Br} & -0.00000000 & 0.72443929 & -0.00000000\end{array}$

$\mathrm{C}_{2} \mathrm{H}_{4}$

D2h symmetry; $\mathrm{E}=\mathbf{- 7 8 . 4 8 4 5 5 7}$

$\begin{array}{lccc}\mathrm{C} & 0.00000000 & 0.00000000 & 0.66422286 \\ \mathrm{H} & 0.00000000 & 0.92423050 & 1.23594478 \\ \mathrm{H} & -0.00000000 & -0.92423050 & 1.23594478 \\ \mathrm{C} & 0.00000000 & 0.00000000 & -0.66422286 \\ \mathrm{H} & -0.00000000 & -0.92423050 & -1.23594478 \\ \mathrm{H} & 0.00000000 & 0.92423050 & -1.23594478\end{array}$

\section{$\mathrm{CH}_{3}-\mathrm{C}_{2} \mathrm{H}_{3}$}

Cs symmetry; $\mathrm{E}=\mathbf{- 1 1 7 . 7 5 8 0 2 8 9}$

$\begin{array}{lccc}\mathrm{C} & 1.28955111 & 0.14350974 & 0.00000000 \\ \mathrm{C} & 0.00000000 & 0.47364347 & 0.00000000 \\ \mathrm{H} & 1.60929637 & -0.89634515 & 0.00000000 \\ \mathrm{H} & 2.07164071 & 0.89689459 & 0.00000000 \\ \mathrm{H} & -0.27182356 & 1.52984629 & 0.00000000 \\ \mathrm{C} & -1.13577383 & -0.49779628 & 0.00000000 \\ \mathrm{H} & -0.77889151 & -1.53172577 & 0.00000000 \\ \mathrm{H} & -1.77644284 & -0.35740576 & 0.87906114 \\ \mathrm{H} & -1.77644284 & -0.35740576 & -0.87906114\end{array}$


$\mathrm{OCH}_{3}-\mathrm{C}_{2} \mathrm{H}_{3}$

Cs symmetry; $\mathrm{E}=\mathbf{- 1 9 2 . 8 8 8 5 1 5 8}$

$\begin{array}{lrrc}\mathrm{C} & -1.32820050 & 0.79373232 & 0.00000000 \\ \mathrm{C} & 0.00000000 & 0.92593980 & 0.00000000 \\ \mathrm{H} & -1.83623380 & -0.16320946 & 0.00000000 \\ \mathrm{H} & -1.94161892 & 1.68620348 & 0.00000000 \\ \mathrm{H} & 0.47914857 & 1.90147474 & 0.00000000 \\ \mathrm{O} & 0.93639587 & -0.04495486 & 0.00000000 \\ \mathrm{C} & 0.45698522 & -1.36984478 & 0.00000000 \\ \mathrm{H} & -0.14947775 & -1.57253385 & 0.89236749 \\ \mathrm{H} & -0.14947775 & -1.57253385 & -0.89236749 \\ \mathrm{H} & 1.33378434 & -2.01872615 & 0.00000000\end{array}$

$\mathrm{NH}_{2}-\mathrm{C}_{2} \mathrm{H}_{3}$

C1 symmetry; $\mathrm{E}=-\mathbf{1 3 3 . 7 8 6 2 5 4 4}$

$\begin{array}{lccc}\mathrm{C} & 0.06563160 & 0.42689883 & -0.00187753 \\ \mathrm{C} & 1.24919207 & -0.19534997 & 0.01730799 \\ \mathrm{H} & 2.16851982 & 0.37638288 & 0.00023377 \\ \mathrm{H} & 1.33064714 & -1.27904193 & 0.02444193 \\ \mathrm{H} & 0.02609562 & 1.51494643 & -0.01452912 \\ \mathrm{~N} & -1.18384826 & -0.16798176 & -0.08701096 \\ \mathrm{H} & -1.92824394 & 0.32884634 & 0.37789699 \\ \mathrm{H} & -1.19902284 & -1.15455457 & 0.12845033\end{array}$

$\mathrm{NO}_{2}-\mathrm{C}_{8} \mathrm{H}_{7}$

Cs symmetry; $\mathrm{E}=\mathbf{- 5 1 3 . 5 7 3 6 6 6 2}$

$\begin{array}{lrrr}\mathrm{C} & -0.52512694 & -4.07797993 & 0.00000000 \\ \mathrm{C} & 0.43125961 & -3.14474157 & 0.00000000 \\ \mathrm{H} & -0.27052000 & -5.13253897 & 0.00000000 \\ \mathrm{H} & -1.58425931 & -3.83734942 & 0.00000000 \\ \mathrm{H} & 1.47048064 & -3.47080181 & 0.00000000 \\ \mathrm{C} & 0.24447544 & -1.69033909 & 0.00000000 \\ \mathrm{C} & 1.37967122 & -0.86520848 & 0.00000000 \\ \mathrm{C} & -1.02156410 & -1.08069039 & 0.00000000 \\ \mathrm{C} & 1.26905288 & 0.51730720 & 0.00000000 \\ \mathrm{C} & 0.00000000 & 1.08063924 & 0.00000000 \\ \mathrm{C} & -1.15057051 & 0.29738000 & 0.00000000 \\ \mathrm{H} & 2.36602145 & -1.32099366 & 0.00000000 \\ \mathrm{H} & -1.91838267 & -1.69158081 & 0.00000000 \\ \mathrm{H} & 2.13842906 & 1.16401212 & 0.00000000 \\ \mathrm{H} & -2.11991445 & 0.78165898 & 0.00000000 \\ \mathrm{~N} & -0.13166390 & 2.53454231 & 0.00000000 \\ \mathrm{O} & 0.89557337 & 3.19453730 & 0.00000000 \\ \mathrm{O} & -1.26099750 & 2.99891214 & 0.00000000\end{array}$


$\mathrm{CF}_{3}-\mathrm{C}_{8} \mathrm{H}_{7}$

C1 symmetry; $\mathrm{E}=\mathbf{- 6 4 6 . 0 0 1 9 8 4 2}$

$\begin{array}{lrrr}\mathrm{C} & 4.43397394 & -0.58195322 & 0.02322925 \\ \mathrm{C} & 3.51693501 & 0.38944281 & 0.01072455 \\ \mathrm{H} & 5.49275888 & -0.34559396 & 0.03477848 \\ \mathrm{H} & 4.17488107 & -1.63682777 & 0.02303049 \\ \mathrm{H} & 3.86162187 & 1.42297545 & 0.01288543 \\ \mathrm{C} & 2.05814036 & 0.23119431 & -0.00466357 \\ \mathrm{C} & 1.25367536 & 1.37826299 & -0.00996018 \\ \mathrm{C} & 1.42242253 & -1.02006152 & -0.01856720 \\ \mathrm{C} & -0.13230579 & 1.28871182 & -0.02470455 \\ \mathrm{C} & -0.74267950 & 0.03842999 & -0.03645044 \\ \mathrm{C} & 0.04059647 & -1.11693779 & -0.03357241 \\ \mathrm{H} & 1.72664510 & 2.35690901 & -0.00490670 \\ \mathrm{H} & 2.01505662 & -1.92949438 & -0.02138113 \\ \mathrm{H} & -0.74067682 & 2.18702425 & -0.03544532 \\ \mathrm{H} & -0.43819007 & -2.09081867 & -0.05286075 \\ \mathrm{C} & -2.23684476 & -0.07603918 & 0.00303727 \\ \mathrm{~F} & -2.83636436 & 1.01136306 & -0.50761317 \\ \mathrm{~F} & -2.68924568 & -0.21885673 & 1.26180596 \\ \mathrm{~F} & -2.67167486 & -1.14256046 & -0.68867102\end{array}$

$\mathrm{Br}-\mathrm{C}_{8} \mathrm{H}_{7}$

Cs symmetry; $\mathrm{E}=\mathbf{- 2 8 7 9 . 7 5 4 1 0 4 3}$

$\begin{array}{lrrr}\mathrm{C} & -0.34256854 & -4.51063034 & 0.00000000 \\ \mathrm{C} & 0.57658737 & -3.54090468 & 0.00000000 \\ \mathrm{H} & -0.04806297 & -5.55476231 & 0.00000000 \\ \mathrm{H} & -1.41022818 & -4.31020829 & 0.00000000 \\ \mathrm{H} & 1.62738300 & -3.82931506 & 0.00000000 \\ \mathrm{C} & 0.34138146 & -2.09309196 & 0.00000000 \\ \mathrm{C} & 1.44193061 & -1.22636180 & 0.00000000 \\ \mathrm{C} & -0.94027433 & -1.52263461 & 0.00000000 \\ \mathrm{C} & 1.28360714 & 0.15453949 & -0.00000000 \\ \mathrm{C} & -0.00000000 & 0.68594992 & -0.00000000 \\ \mathrm{C} & -1.11655715 & -0.14700538 & 0.00000000 \\ \mathrm{H} & 2.44606128 & -1.64319942 & 0.00000000 \\ \mathrm{H} & -1.81891811 & -2.16052454 & 0.00000000 \\ \mathrm{H} & 2.14611076 & 0.81186828 & -0.00000000 \\ \mathrm{H} & -2.11254311 & 0.28223944 & 0.00000000 \\ \mathrm{Br} & -0.23698406 & 2.56013537 & 0.00000000\end{array}$

\section{$\mathrm{H}-\mathrm{C}_{8} \mathrm{H}_{7}$}

Cs symmetry; $\mathrm{E}=\mathbf{- 3 0 9 . 2 7 6 4 1 4 7}$

$\begin{array}{lrrr}\mathrm{C} & -1.48083345 & 2.59183782 & 0.00000000 \\ \mathrm{C} & -0.28322751 & 1.99933480 & 0.00000000 \\ \mathrm{H} & -1.56572849 & 3.67351557 & 0.00000000 \\ \mathrm{H} & -2.41301519 & 2.03405277 & 0.00000000 \\ \mathrm{H} & 0.60248133 & 2.63455414 & 0.00000000 \\ \mathrm{C} & -0.00000000 & 0.55911090 & 0.00000000 \\ \mathrm{C} & 1.33481979 & 0.13289520 & 0.00000000 \\ \mathrm{C} & -1.00474995 & -0.42021361 & 0.00000000 \\ \mathrm{C} & 1.66021179 & -1.21942458 & 0.00000000 \\ \mathrm{C} & 0.65158687 & -2.17769187 & 0.00000000 \\ \mathrm{C} & -0.68245616 & -1.77027690 & 0.00000000 \\ \mathrm{H} & 2.12667342 & 0.87842124 & 0.00000000 \\ \mathrm{H} & -2.04927469 & -0.12263683 & 0.00000000 \\ \mathrm{H} & 2.70295387 & -1.52414081 & 0.00000000 \\ \mathrm{H} & -1.47621752 & -2.51215391 & 0.00000000 \\ \mathrm{H} & 0.90001900 & -3.23504280 & 0.00000000\end{array}$


$\mathrm{CH}_{3}-\mathrm{C}_{8} \mathrm{H}_{7}$

Cs symmetry; $\mathrm{E}=\mathbf{- 3 4 8 . 5 4 8 0 6 3 9}$

$\begin{array}{lrrr}\mathrm{C} & -1.22293260 & 3.25218077 & 0.00000000 \\ \mathrm{C} & -0.10629174 & 2.51785763 & 0.00000000 \\ \mathrm{H} & -1.17567115 & 4.33614909 & 0.00000000 \\ \mathrm{H} & -2.21597651 & 2.81166439 & 0.00000000 \\ \mathrm{H} & 0.85022163 & 3.04065681 & 0.00000000 \\ \mathrm{C} & 0.00000000 & 1.05507522 & 0.00000000 \\ \mathrm{C} & 1.26807697 & 0.46376714 & 0.00000000 \\ \mathrm{C} & -1.11403040 & 0.20090936 & 0.00000000 \\ \mathrm{C} & 1.42212697 & -0.91878077 & 0.00000000 \\ \mathrm{C} & 0.31387385 & -1.76530858 & 0.00000000 \\ \mathrm{C} & -0.95746786 & -1.17561017 & 0.00000000 \\ \mathrm{H} & 2.14934316 & 1.10129362 & 0.00000000 \\ \mathrm{H} & -2.11635729 & 0.61954442 & 0.00000000 \\ \mathrm{H} & 2.42171352 & -1.34701486 & 0.00000000 \\ \mathrm{H} & -1.83922458 & -1.81278766 & 0.00000000 \\ \mathrm{C} & 0.46652257 & -3.25993133 & 0.00000000 \\ \mathrm{H} & -0.00632554 & -3.70821350 & 0.88100409 \\ \mathrm{H} & -0.00632554 & -3.70821350 & -0.88100409 \\ \mathrm{H} & 1.51933560 & -3.55403436 & 0.00000000\end{array}$

\section{$\mathrm{OCH}_{3}-\mathrm{C}_{8} \mathrm{H}_{7}$}

Cs symmetry; $\mathrm{E}=\mathbf{- 4 2 3 . 6 7 6 6 4 2 2}$

$\begin{array}{lrrr}\mathrm{C} & -3.54296565 & -1.51432160 & 0.00000000 \\ \mathrm{C} & -2.25791957 & -1.88277877 & 0.00000000 \\ \mathrm{H} & -4.33411343 & -2.25669126 & 0.00000000 \\ \mathrm{H} & -3.85655975 & -0.47407068 & 0.00000000 \\ \mathrm{H} & -2.03361053 & -2.94965134 & 0.00000000 \\ \mathrm{C} & -1.07640041 & -1.01567737 & 0.00000000 \\ \mathrm{C} & 0.20148144 & -1.59761740 & 0.00000000 \\ \mathrm{C} & -1.14422050 & 0.38204830 & 0.00000000 \\ \mathrm{C} & 1.35238683 & -0.82893105 & 0.00000000 \\ \mathrm{C} & 1.26105032 & 0.56617490 & 0.00000000 \\ \mathrm{C} & 0.00000000 & 1.17086115 & 0.00000000 \\ \mathrm{H} & 0.28841486 & -2.68167875 & 0.00000000 \\ \mathrm{H} & -2.11210129 & 0.87510453 & 0.00000000 \\ \mathrm{H} & 2.33762117 & -1.28403195 & 0.00000000 \\ \mathrm{H} & -0.10010126 & 2.25047516 & 0.00000000 \\ \mathrm{O} & 2.43760599 & 1.23867807 & 0.00000000 \\ \mathrm{C} & 2.39122338 & 2.64609382 & 0.00000000 \\ \mathrm{H} & 1.88669900 & 3.03586711 & 0.89368649 \\ \mathrm{H} & 3.42838934 & 2.98427351 & 0.00000000 \\ \mathrm{H} & 1.88669900 & 3.03586711 & -0.89368649\end{array}$


$\mathrm{NH}_{2}-\mathrm{C}_{8} \mathrm{H}_{7}$

C1 symmetry; $\mathrm{E}=\mathbf{- 3 6 4 . 5 7 6 8 7 6 6}$

$\begin{array}{lrrr}\mathrm{C} & -3.42589160 & -0.47951427 & 0.00646990 \\ \mathrm{C} & -2.45840101 & 0.44399154 & 0.00633250 \\ \mathrm{H} & -2.75661535 & 1.49286192 & 0.01112846 \\ \mathrm{H} & -3.22007622 & -1.54632119 & 0.00086429 \\ \mathrm{H} & -4.47135947 & -0.18968196 & 0.01149228 \\ \mathrm{C} & -1.01073376 & 0.22768921 & -0.00037645 \\ \mathrm{C} & -0.14874755 & 1.33230573 & -0.00203095 \\ \mathrm{C} & -0.41659396 & -1.04379814 & -0.00357443 \\ \mathrm{C} & 1.23057690 & 1.18861859 & -0.00530452 \\ \mathrm{C} & 1.81154906 & -0.08617836 & -0.00779233 \\ \mathrm{C} & 0.95797933 & -1.20196383 & -0.00747853 \\ \mathrm{H} & -0.57445085 & 2.33334673 & 0.00177862 \\ \mathrm{H} & -1.04289868 & -1.93142853 & -0.00081868 \\ \mathrm{H} & 1.86860102 & 2.06924714 & -0.01042869 \\ \mathrm{H} & 1.38733865 & -2.20127191 & -0.01477647 \\ \mathrm{~N} & 3.18642927 & -0.24590603 & -0.06504950 \\ \mathrm{H} & 3.72951429 & 0.53643281 & 0.26556334 \\ \mathrm{H} & 3.53651741 & -1.12874550 & 0.27307225\end{array}$

$\left[\mathrm{Au}\left(\mathrm{C}_{2} \mathrm{H}_{4}\right)\right]^{+}$

C2v symmetry; $\mathrm{E}=\mathbf{- 2 1 3 . 9 4 8 6 3 0 3}$

$\begin{array}{crrr}\mathrm{Au} & 0.00000000 & 0.00000000 & 0.35466115 \\ \mathrm{C} & 0.00000000 & 0.69986557 & -1.71831245 \\ \mathrm{C} & -0.00000000 & -0.69986557 & -1.71831245 \\ \mathrm{H} & 0.92882355 & -1.25342590 & -1.84962043 \\ \mathrm{H} & -0.92882355 & -1.25342590 & -1.84962043 \\ \mathrm{H} & 0.92882355 & 1.25342590 & -1.84962043 \\ \mathrm{H} & -0.92882355 & 1.25342590 & -1.84962043\end{array}$


[( $\left.\mathrm{NH}_{2} \mathbf{N H C}\right) \mathrm{AuCl}$

C1 symmetry; $\mathrm{E}=\mathbf{- 1 4 9 5 . 7 4 9 0 5 1 7}$

\begin{tabular}{|c|c|c|c|}
\hline $\mathrm{Au}$ & 0.15080280 & 1.39320794 & -0.08661579 \\
\hline $\mathrm{C}$ & 0.02633133 & -0.58611033 & 0.02707855 \\
\hline $\mathrm{C}$ & 0.54665914 & -2.78642208 & 0.17065858 \\
\hline $\mathrm{C}$ & -0.81156519 & -2.69219737 & 0.16488390 \\
\hline $\mathrm{C}$ & 2.30729682 & -1.27488702 & -2.47116478 \\
\hline $\mathrm{C}$ & -2.50616150 & -1.31313103 & -2.41279728 \\
\hline $\mathrm{C}$ & -2.20600744 & -0.33824221 & 2.53843809 \\
\hline $\mathrm{C}$ & 2.34627716 & -0.97580486 & 2.57137245 \\
\hline $\mathrm{C}$ & 2.42273313 & -1.15209645 & 0.05107533 \\
\hline $\mathrm{C}$ & 3.08468822 & -0.93373171 & 1.26514092 \\
\hline $\mathrm{C}$ & 3.06543460 & -1.07932701 & -1.19049823 \\
\hline $\mathrm{C}$ & 4.44801636 & -0.64099613 & 1.21323314 \\
\hline $\mathrm{C}$ & 4.42923756 & -0.78344879 & -1.19414246 \\
\hline $\mathrm{C}$ & 5.11481807 & -0.56776096 & -0.00433112 \\
\hline $\mathrm{C}$ & -2.44558324 & -0.82099893 & 0.06417169 \\
\hline $\mathrm{C}$ & -2.99966839 & -0.35477518 & 1.26439807 \\
\hline $\mathrm{C}$ & -3.14687565 & -0.81729332 & -1.14975514 \\
\hline $\mathrm{C}$ & -4.30839176 & 0.12992094 & 1.22485695 \\
\hline $\mathrm{C}$ & -4.45144838 & -0.32335831 & -1.14027242 \\
\hline $\mathrm{C}$ & -5.02689026 & 0.14618357 & 0.03530894 \\
\hline $\mathrm{N}$ & 1.02835937 & -1.48158410 & 0.08102249 \\
\hline $\mathrm{N}$ & -1.11112341 & -1.34099079 & 0.07730716 \\
\hline $\mathrm{H}$ & 1.20530849 & -3.63796412 & 0.21494003 \\
\hline $\mathrm{H}$ & -4.75975219 & 0.50456497 & 2.13928165 \\
\hline $\mathrm{H}$ & -5.01446108 & -0.30200855 & -2.06912589 \\
\hline $\mathrm{H}$ & -6.04238920 & 0.53127738 & 0.02329491 \\
\hline $\mathrm{H}$ & 4.98541193 & -0.46296368 & 2.14056694 \\
\hline $\mathrm{H}$ & 4.95213507 & -0.71678609 & -2.14417015 \\
\hline $\mathrm{H}$ & 6.17591655 & -0.33694387 & -0.02628647 \\
\hline $\mathrm{H}$ & 1.64666212 & -0.13521284 & 2.64294021 \\
\hline $\mathrm{H}$ & 1.76143946 & -1.89507268 & 2.68015060 \\
\hline $\mathrm{H}$ & 3.04138947 & -0.91045862 & 3.41140218 \\
\hline $\mathrm{H}$ & -1.69762775 & -1.29092196 & 2.72004669 \\
\hline $\mathrm{H}$ & -1.43179418 & 0.43657352 & 2.49412623 \\
\hline $\mathrm{H}$ & -2.85155810 & -0.12529994 & 3.39347611 \\
\hline $\mathrm{H}$ & -3.17862643 & -1.18210183 & -3.26335801 \\
\hline $\mathrm{H}$ & -1.58133520 & -0.76481967 & -2.62142668 \\
\hline $\mathrm{H}$ & -2.25116666 & -2.37603727 & -2.33832388 \\
\hline $\mathrm{H}$ & 1.71185029 & -2.19354151 & -2.45736786 \\
\hline $\mathrm{H}$ & 1.61488718 & -0.44161299 & -2.63710130 \\
\hline $\mathrm{H}$ & 2.99053160 & -1.32165259 & -3.32209608 \\
\hline $\mathrm{N}$ & -1.81215189 & -3.64003551 & 0.14365749 \\
\hline $\mathrm{H}$ & -1.53648737 & -4.53921025 & 0.50810412 \\
\hline $\mathrm{H}$ & -2.68653678 & -3.31879203 & 0.53808993 \\
\hline $\mathrm{Cl}$ & 0.29198765 & 3.68474667 & -0.20378570 \\
\hline
\end{tabular}


[(NO$\left.{ }_{2} \mathrm{NHC}\right) \mathrm{AuCl}$

Cs symmetry; $\mathrm{E}=\mathbf{- 1 6 4 4 . 7 3 0 2 1 7 1}$

$\begin{array}{lrrr}\mathrm{Au} & 1.52227670 & 0.47280572 & 0.00000000 \\ \mathrm{C} & -0.40840209 & 0.03555586 & 0.00000000 \\ \mathrm{C} & -2.65125800 & 0.27616863 & 0.00000000 \\ \mathrm{C} & -2.36352629 & -1.05208100 & 0.00000000 \\ \mathrm{C} & -1.26011339 & 2.25748308 & 2.52915228 \\ \mathrm{C} & -0.26312929 & -2.32962281 & 2.52631120 \\ \mathrm{C} & -0.26312929 & -2.32962281 & -2.52631120 \\ \mathrm{C} & -1.26011339 & 2.25748308 & -2.52915228 \\ \mathrm{C} & -1.29696358 & 2.35319807 & 0.00000000 \\ \mathrm{C} & -1.22928397 & 3.01310780 & -1.23238908 \\ \mathrm{C} & -1.22928397 & 3.01310780 & 1.23238908 \\ \mathrm{C} & -1.09955472 & 4.40205499 & -1.20637894 \\ \mathrm{C} & -1.09955472 & 4.40205499 & 1.20637894 \\ \mathrm{C} & -1.03663714 & 5.08973292 & 0.00000000 \\ \mathrm{C} & -0.20906259 & -2.40874672 & 0.00000000 \\ \mathrm{C} & 0.16320649 & -2.95557703 & -1.23111536 \\ \mathrm{C} & 0.16320649 & -2.95557703 & 1.23111536 \\ \mathrm{C} & 0.95016888 & -4.10676555 & -1.20580468 \\ \mathrm{C} & 0.95016888 & -4.10676555 & 1.20580468 \\ \mathrm{C} & 1.34143059 & -4.67661152 & 0.00000000 \\ \mathrm{~N} & -1.44847456 & 0.92305116 & 0.00000000 \\ \mathrm{~N} & -0.98502811 & -1.19184458 & 0.00000000 \\ \mathrm{H} & -3.60432754 & 0.78040652 & 0.00000000 \\ \mathrm{H} & 1.25910350 & -4.55423536 & -2.14609829 \\ \mathrm{H} & 1.25910350 & -4.55423536 & 2.14609829 \\ \mathrm{H} & 1.95728185 & -5.57097286 & 0.00000000 \\ \mathrm{H} & -1.04063237 & 4.94350008 & -2.14615169 \\ \mathrm{H} & -1.04063237 & 4.94350008 & 2.14615169 \\ \mathrm{H} & -0.93294745 & 6.17069981 & 0.00000000 \\ \mathrm{H} & -0.37183970 & 1.62296855 & -2.62773856 \\ \mathrm{H} & -2.13797150 & 1.60724636 & -2.60813207 \\ \mathrm{H} & -1.27587517 & 2.94678530 & -3.37577436 \\ \mathrm{H} & -1.35488948 & -2.29112526 & -2.61321497 \\ \mathrm{O} & 0.11616898 & -1.30556538 & -2.61557716 \\ \mathrm{H} & -2.11749111 & -2.90295082 & -3.37402023 \\ \mathrm{H} & 0.11749111 & -2.90295082 & 3.37402023 \\ \mathrm{H} & 0.11616898 & -1.30556538 & 2.61557716 \\ \mathrm{H} & -1.35488948 & -2.29112526 & 2.61321497 \\ \mathrm{H} & -2.13797150 & 1.60724636 & 2.60813207 \\ \mathrm{H} & -0.37183970 & 1.62296855 & 2.62773856 \\ \mathrm{H} & -1.27587517 & 2.94678530 & 3.37577436 \\ \mathrm{H} & -3.31991799 & -2.11207689 & 0.00000000 \\ \mathrm{H} & -4.49285002 & -1.76794600 & 0.00000000 \\ \mathrm{H} & & -3.25892830 & 0.00000000 \\ \mathrm{H} & -141495 & 0.99674064 & 0.00000000\end{array}$

